Portland State University

PDXScholar

Dissertations and Theses

Dissertations and Theses

Winter 3-1-2012

\title{
Application of Inter-Die Rank Statistics in Defect Detection
}

Vivek Bakshi

Portland State University

Follow this and additional works at: https://pdxscholar.library.pdx.edu/open_access_etds

Part of the Electrical and Computer Engineering Commons, Other Computer Sciences Commons, and the Other Statistics and Probability Commons

Let us know how access to this document benefits you.

\section{Recommended Citation}

Bakshi, Vivek, "Application of Inter-Die Rank Statistics in Defect Detection" (2012). Dissertations and Theses. Paper 90.

https://doi.org/10.15760/etd.90

This Thesis is brought to you for free and open access. It has been accepted for inclusion in Dissertations and Theses by an authorized administrator of PDXScholar. Please contact us if we can make this document more accessible: pdxscholar@pdx.edu. 
Application of Inter-Die Rank Statistics in Defect Detection

by

Vivek Bakshi

A thesis submitted in partial fulfillment of the requirements for the degree of

Master of Science

in

Electrical and Computer Engineering

Thesis Committee:

W. Robert Daasch, Chair

C. Glenn Shirley

James E. Morris

Portland State University

2012 


\begin{abstract}
This thesis presents a statistical method to identify the test escapes. Test often acquires parametric measurements as a function of logical state of a chip. The usual method of classifying chips as pass or fail is to compare each state measurement to a test limit. Subtle manufacturing defects are escaping the test limits due to process variations in deep sub-micron technologies which results in mixing of healthy and faulty parametric test measurements. This thesis identifies the chips with subtle defects by using rank order of the parametric measurements. A hypothesis is developed that a defect is likely to disturb the defect-free ranking, whereas a shift caused by process variations will not affect the rank. The hypothesis does not depend on a-priori knowledge of a defect-free ranking of parametric measurements.
\end{abstract}

This thesis introduces a modified Estimation Maximization (EM) algorithm to separate the healthy and faulty tau components calculated from parametric responses of die pairs on a wafer. The modified EM uses generalized beta distributions to model the two components of tau mixture distribution. The modified EM estimates the faulty probability of each die on a wafer.

The sensitivity of the modified EM is evaluated using Monte Carlo simulations. The modified EM is applied on production product A. An average $30 \%$ reduction in DPPM (defective parts per million) is observed in Product A across all lots. 


\section{Acknowledgements}

This is a great opportunity for me to thank everyone who directly or indirectly helped in completion of my thesis. First of all, I would like to thank my graduate advisor Dr. Robert Daasch who constantly drove me towards perfection. His thorough knowledge of circuit design and test cleared my circuit fundamentals. He was always available whenever I needed help. His guidance and encouragement has taught me important lessons in my life. I would also like to thank Dr. Glenn Shirley whose extremely helpful reviews helped my research. His constant help during the research enabled me to arrange my thoughts and ideas into a presentable format. I am grateful to Dr. James E. Morris for agreeing to serve on my thesis committee.

I am also indebted to my colleagues and friends without which this thesis would not have been possible. Special thanks goes to ICDT colleagues Kapil, Rohan, Vedanth, Chaitrali and Kalyan. I would also like to thank my friends Neera, Rajesh, Aniket, Shubham and Prashant for giving me support.

I thank my parents and brother for the enormous support they have given me during two and a half years of my school life at Portland State University. They were always besides me in both happy and tough times. I would also like to take a moment to remember my uncle, aunt and grandfather who died during my stay in the U.S. Their memories will always remain in my heart.

Special thanks to my cousin Sidd who always cheers me up. 


\section{Contents}

Abstract

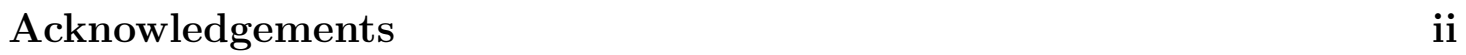

$\begin{array}{ll}\text { List of Tables } & \text { V }\end{array}$

List of Figures vi vi

1 Introduction, Motivation and Contribution 1

1.1 Introduction . . . . . . . . . . . . . . . . . . . . . 1

1.2 Parametric Tests . . . . . . . . . . . . . . . . 3

$1.2 .1 \quad$ IDDQ test $\ldots \ldots \ldots \ldots \ldots$

1.2 .2 Path delay test . . . . . . . . . . . . . . . . . . 4

1.2 .3 MinVdd test . . . . . . . . . . . . . . . . . . 5

1.3 Figures of Merit . . . . . . . . . . . . . . . . . . . . 5

1.4 Thesis Motivation . . . . . . . . . . . . . . . . . . . 7

1.5 Thesis Contributions . . . . . . . . . . . . . . . . . 9

1.6 Thesis Organization . . . . . . . . . . . . . . . . . . 10

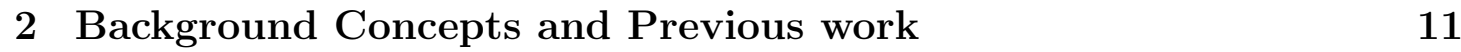

2.1 Rank Order . . . . . . . . . . . . . . . . . . . . 11

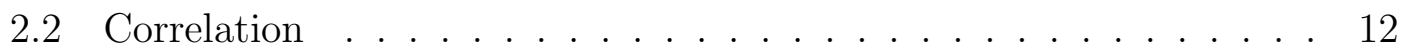

2.3 Kendall's Tau Correlation . . . . . . . . . . . . . . . . . . . . 12

2.3 .1 Graphical Representation of Tau . . . . . . . . . . . 13 
2.4 IDDQ Current Signature . . . . . . . . . . . . . . . 15

2.5 Previous Work $\ldots \ldots \ldots \ldots$

3 Defect Detection Model 22

3.1 Kendall's Tau Calculation . . . . . . . . . . . . . . . . . . 22

3.2 Correlation Model . . . . . . . . . . . . . . . . . . . . . . . . . . . . 23

3.3 Correlation Mixture Model Building . . . . . . . . . . . . . . . . . . 27

3.3.1 Estimation Maximization (EM) algorithm . . . . . . . . . . 29

3.4 Monte Carlo Simulations . . . . . . . . . . . . . . . . . . . . 33

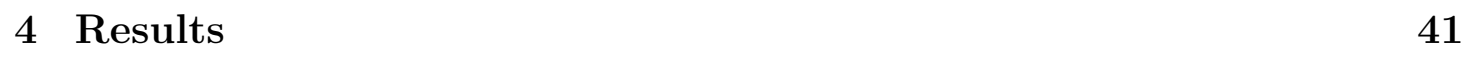

4.1 Monte Carlo Simulation Results . . . . . . . . . . . . . . . . . . . . 41

4.1 .1 Without Noise . . . . . . . . . . . . . . . . . 41

4.1 .2 With Noise Model . . . . . . . . . . . . . . . . . . . . . . . 44

$4.2 \quad$ Application on Production Data . . . . . . . . . . . . . . . . . . . . 52

$4.2 .1 \quad$ Data Description . . . . . . . . . . . . . . . . . 52

$4.2 .2 \quad$ Defective Probability Plots . . . . . . . . . . . . . . . 53

4.2 .3 Key Figures of Merit . . . . . . . . . . . . . . . . . . . . . 59

4.2 .4 Effect of Reduced IDDQ Input States . . . . . . . . . . . . . 66

$\begin{array}{lll}5 & \text { Conclusion and Recommendations } & 74\end{array}$

5.1 Conclusion . . . . . . . . . . . . . . . . . . . 74

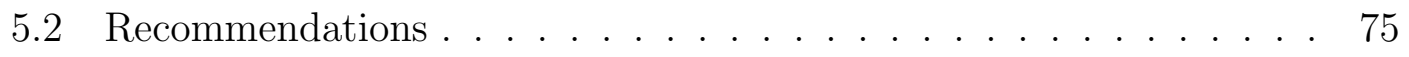

\begin{tabular}{ll}
\hline References & 78
\end{tabular} 


\section{List of Tables}

$4.1 \quad$ Kill ratio of Lot 1 for probability threshold $=0.95$. Total $\mathrm{KR}=1 / 9$. 63

4.2 Kill ratio of Lot 2 for probability threshold $=0.95$. Total $\mathrm{KR}=1 / 5.63$

4.3 Kill ratio of Lot 3 for probability threshold $=0.95$. Total $\mathrm{KR}=1 / 5$. 64

4.4 Kill ratio of Lot 4 for probability threshold $=0.95$. Total $\mathrm{KR}=1 / 0$. 64

4.5 Kill ratio of Lot 1 for probability threshold $=0.80$. Total $\mathrm{KR}=1 / 17$. 64

4.6 Kill ratio of Lot 2 for probability threshold $=0.80$. Total $\mathrm{KR}=1 / 6$. 65

$4.7 \quad$ Kill ratio of Lot 3 for probability threshold $=0.80$. Total $\mathrm{KR}=1 / 5$. 65

4.8 Kill ratio of Lot 4 for probability threshold $=0.80$. Total $\mathrm{KR}=1 / 1$. 65 


\section{List of Figures}

1.1 Typical I.C test flow. $\ldots \ldots \ldots \ldots \ldots \ldots$

1.2 Example of IDDQ test on a bridging defect. . . . . . . . . . . 4

$1.3 \quad$ An example circuit showing the critical path P. . . . . . . . 5

1.4 Figures of merit. $\ldots \ldots \ldots \ldots \ldots \ldots$

1.5 Illustration showing the parametric variation taking place within a wafer and a lot for four input states. Bars represent process variation of state dependent parametric test values. Dot represents the test value of a die for each of the four states. $\ldots \ldots \ldots \ldots$

2.1 Discordance plot showing correlation between two measurements. The labels are the state numbers. . . . . . . . . . . . . . . . 14

2.2 Difference between IDDQ for input states of a defect-free circuit and a defective circuit. . . . . . . . . . . . . . . 16

2.3 IDDQ plots of a defect free die. $\ldots \ldots \ldots \ldots \ldots$

$2.4 \quad$ IDDQ plots of a defective die. $\ldots \ldots \ldots \ldots \ldots \ldots$

3.1 Tau matrix of die pairs. $\ldots \ldots \ldots \ldots \ldots \ldots$

$3.2 \quad$ Correlation model showing inter-die tau distributions calculated using die pairs. $\ldots \ldots \ldots \ldots \ldots \ldots \ldots \ldots \ldots$

$3.3 \quad$ Correlation model mixture of synthesized healthy-healthy, healthyfaulty and faulty-healthy distributions. The healthy-healthy distribution is shown by rightmost red curve while the healthy-faulty and faulty-healthy distributions is shown by leftmost blue curve. . . . . 26 
3.4 Tau mixture histogram calculated from a wafer of the production 90nm dataset. . . . . . . . . . . . . . . . . . . . 27

3.5 Flow chart of modified EM algorithm. The box in dashed lines shows the modified step in the EM algorithm. . . . . . . . . . . . . 30

$3.6 \quad$ Two component synthetic beta mixture generator. Bold tau values are healthy component tau values while underlines tau values are faulty component tau values. . . . . . . . . . . . . . . . . . . . 35

3.7 Two component mixtures showing examples of varying mixing proportion of healthy and faulty components. . . . . . . . . . . . 37

3.8 Beta mixture model showing examples of effect of yield on proportion of healthy and faulty component distributions. . . . . . . . . . 38

3.9 An example mixture of healthy and faulty component distributions showing where a contaminant distribution is placed (dotted lines). Simulated parameters are $\alpha_{H}=18, \beta_{H}=9, \alpha_{F}=3, \alpha_{H}=9$, $p=0.64, \alpha_{C}=3, \beta_{C}=3$.

4.1 Comparison between input $\alpha$ 's and $\beta$ 's and estimated $\alpha$ 's and $\beta$ 's. . 42

$4.2 \quad$ Comparison between input $\alpha$ 's and $\beta$ 's and estimated $\alpha$ 's and $\beta$ 's. . 43

4.3 Comparison between input yield and estimated yield. . . . . . . . . 44

4.4 Tau histograms with and without noise. Simulated parameters are $\alpha_{H}=18, \beta_{H}=9, \alpha_{F}=3, \alpha_{H}=9, p=0.64, \alpha_{C}=3, \beta_{C}=3$. . . . 46

4.5 Tau histogram with maximum noise factor, $\mathrm{C}=0.5$. Simulated parameters are $\alpha_{H}=18, \beta_{H}=9, \alpha_{F}=3, \alpha_{H}=9, p=0.64$, $\alpha_{C}=3, \beta_{C}=3 . \ldots \ldots \ldots \ldots \ldots$. . . . . . . . . . . 47

4.6 Comparison between input yield and estimated yield in presence of noise with $\mathrm{C}=0.31$. The Figure 4.6 can be compared to Figure 4.3. 48 
4.7 Comparison between input yield and estimated yield in presence of

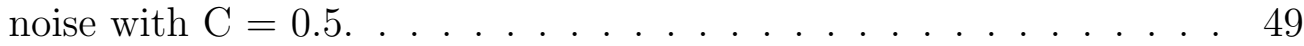

4.8 Comparison between input $\alpha$ 's and $\beta$ 's and estimated $\alpha$ 's and $\beta$ 's with $\mathrm{C}=0.5$. . . . . . . . . . . . . . . . . 50

4.9 Comparison between input $\alpha$ 's and $\beta$ 's and estimated $\alpha$ 's and $\beta$ 's with $\mathrm{C}=0.5$. . . . . . . . . . . . . . . . . . . . 51

4.10 Tau histograms of two example wafers from Product A. . . . . . . . 54

4.11 Tau histogram of an example wafer from Product B. . . . . . . . . 55

4.12 Defective probability plots of two example wafers of Product A. Triangles are $85^{\circ} \mathrm{C}$ wafer-sort passes while squares are $85^{\circ} \mathrm{C}$ wafersort fails. . . . . . . . . . . . . . . . . . . . . 56

4.13 Defective probability plots of two wafers of Product B. Triangles are wafer-sort passes while squares are wafer-sort fails. . . . . . . . . . . 58

4.14 Figure illustrating the original and modified test flow. . . . . . . . 60

4.15 Calculating the figures of merit of modified EM method using 2 by 2 contingency table. . . . . . . . . . . . . . . 61

4.16 DPPM reduction, increase in overkill and yield loss of Lots 1-4. . . 67

4.17 Tau distributions showing comparing the overlap of healthy and faulty components using 64 IDDQ states and 16 IDDQ states. . . . 69

4.18 Discordance plots of test ranks using 1st and 2nd group of eight IDDQ states of two dies from lot 1 in Product A. . . . . . . . . . . 70 4.19 Discordance plots of test ranks using 3rd and 4th group of eight IDDQ states of two dies from lot 1 in Product A. . . . . . . . . . . 71 
4.20 DPPM reduction plots using defective die probabilities obtained from a reduced subset of IDDQ states. The dies above the probability threshold 0.80 and 0.95 are tagged as defective. . . . . . . . 73

5.1 Variation of path delays of 7 different critical paths on a die across wafer $[1] . \ldots \ldots \ldots \ldots \ldots \ldots$. . . . . . . . . . . . . . . . . . . . 


\section{Chapter 1}

\section{Introduction, Motivation and Contribution}

\section{$1.1 \quad$ Introduction}

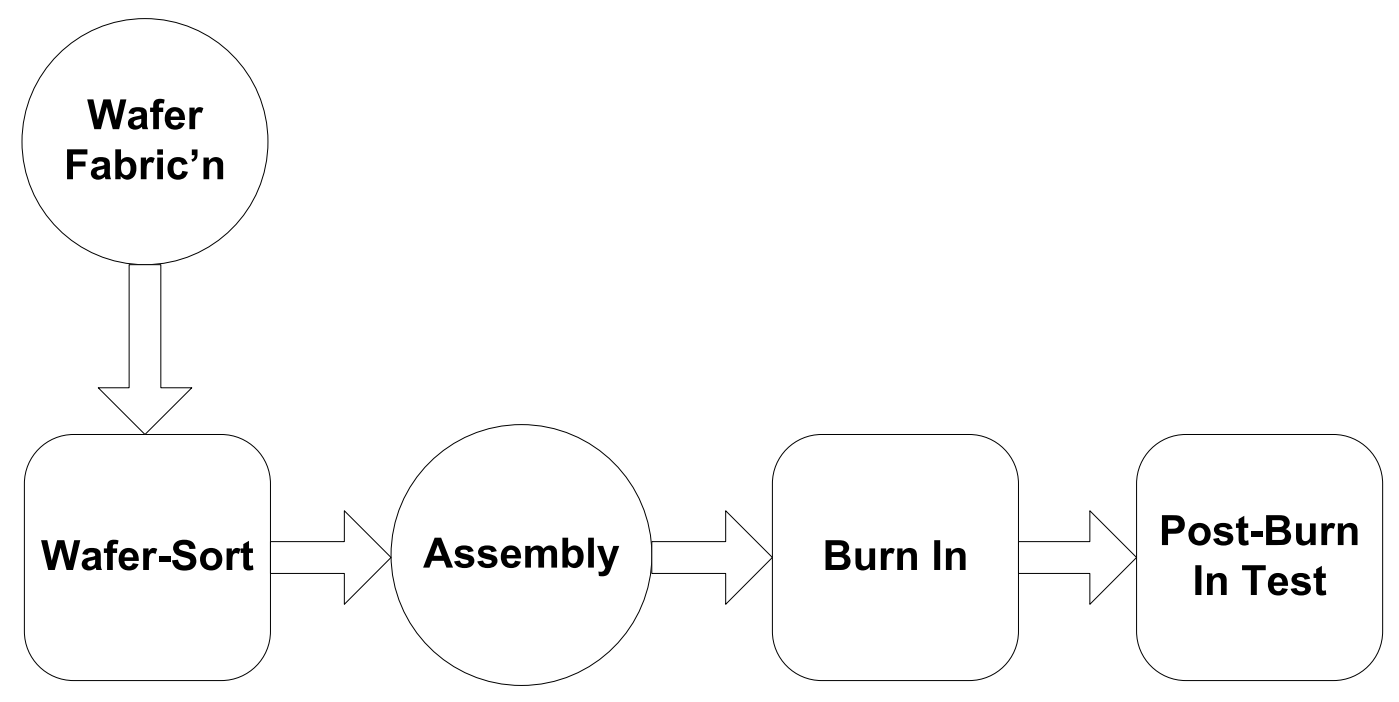

Figure 1.1: Typical I.C test flow.

Figure 1.1 shows a typical I.C test flow involved in the testing of an integrated circuit. The manufacturing steps of an I.C are shown by round boxes. The test modules (e.g wafer-sort and post burn in test) are shown in square boxes. Each test module contains a series of tests applied on parts to ensure elimination of parts having defects. Parts passing each test are sent to downstream tests. Parts are rejected when they fail a test. The I.C test flow is described as follows: 
1. Wafer fabrication: A wafer is fabricated in a manufacturing plant. Fabrication is the source of silicon defects.

2. Wafer-sort: The dies are tested on the wafer. The testing module is called a wafer-sort [2]. Wafer-sort tests are sometimes conducted at both high and low temperatures to ensure the elimination of temperature sensitive defects [3].

3. Assembly: The dies are sliced from the wafers and passing dies are assembled in packages. This step is also a source of package assembly defects such as opens and shorts.

4. Burn In: The dies are stressed under accelerated temperature and voltage conditions for long periods of time [4]. The burn in ensures the elimination of packaged dies which have a high probability to fail during their early life of operation.

5. Post-Burn In test: The packaged dies are subjected to a final screen to test for data-sheet specifications. Parts are tested to ensure correct operation.

Two types of tests are run in each testing module: parametric tests and functional tests. The functional tests are run to verify the operation of the integrated circuit. The functional tests involve giving input binary values to integrated circuit and checking for corresponding logical responses. This thesis discusses about the usage of parametric test data as a screen. The functional tests will not be further discussed in the thesis. 


\subsection{Parametric Tests}

In order to make a parametric test measurement, the chip must be put in a particular logical state [5]. The state of a circuit is the binary values of the logical nodes in the design. The parametric tests are run to check whether the integrated circuit meets specifications, for example, rise and fall times, noise margin, IDDQ, path delay and minVdd specifications [2]. Examples of three state dependent parametric measurements are described in following sections.

\subsubsection{IDDQ test}

The IDDQ test is a state dependent parametric test which measures the leakage current flowing from Vdd to ground in a CMOS circuit. When the circuit is in a steady state, a small amount of leakage current flows in the circuit due to background leakage mechanisms. A defect will draw a high IDDQ current from the power supply compared to the normal leakage current due to some kind of short occurring in the circuit. The types of potential problems detected by IDDQ include process flaws such as bridging defects, mask problems, improper etching and stuck-at faults 6$]$.

Figure 1.2 shows a resistive bridging short between two neighbouring lines driven by two inverters. When the input logic to both the inverters is same, the current flowing from Vdd to ground is equal to background leakage current in CMOS circuits. When the inverters are driven to opposite logic values, an elevated current flows from Vdd to ground due to the bridging short between the two lines. The inputs 00 and 11 do not activate the defect. The inputs 00 and 11 put the circuit in non elevated states. The inputs 10 and 01 activate the defect and put the circuit 
in elevated states.
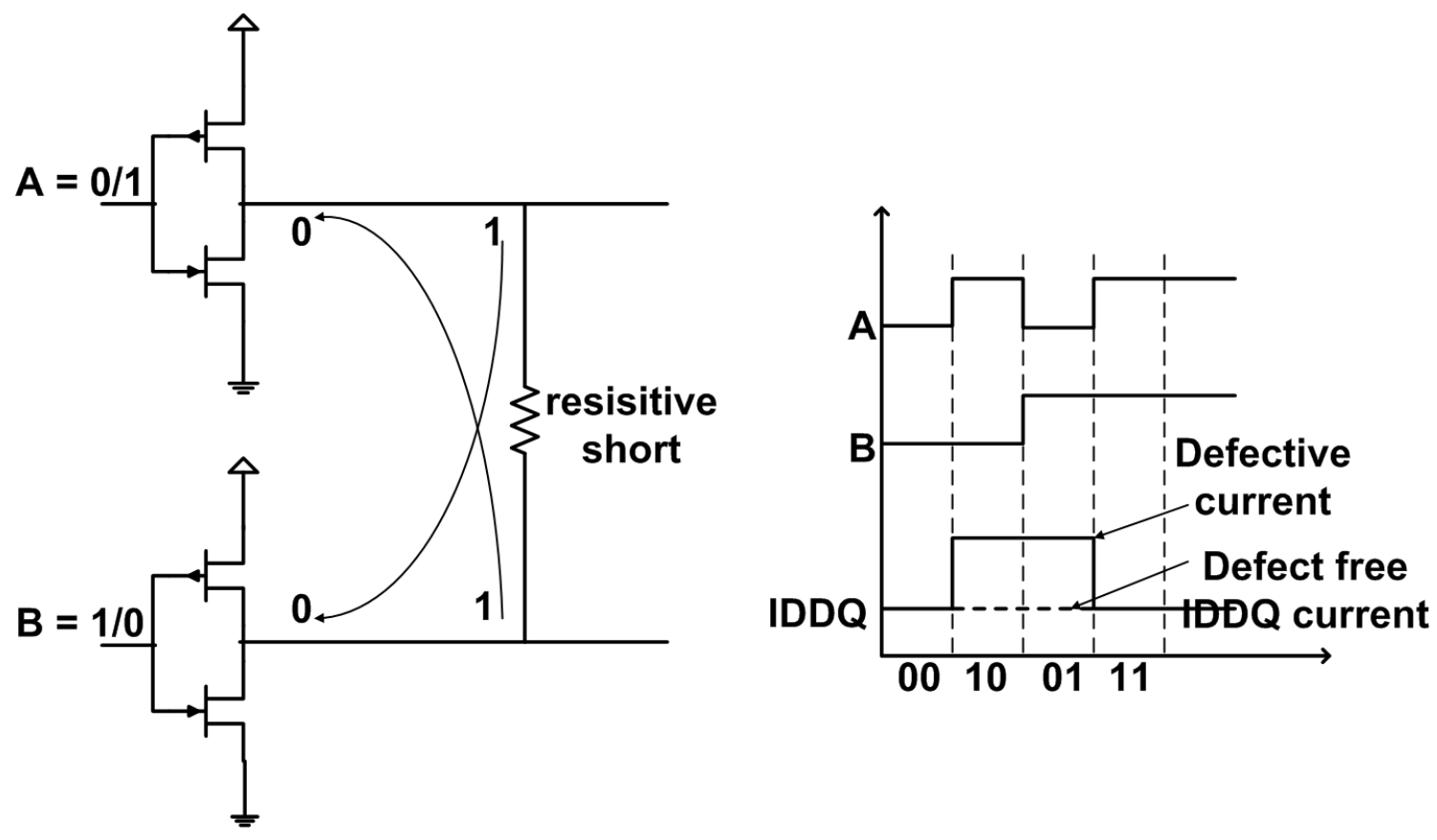

Figure 1.2: Example of IDDQ test on a bridging defect.

\subsubsection{Path delay test}

The path delay test is a state dependent parametric test which checks for defects causing logic circuits to malfunction at the desired clock period [7]. The path delay test requires an input state pair to cause a transition at the output from one state to another state. For a circuit to function correctly, the maximum delay of one or more paths should not exceed the clock period.

Figure 1.3 shows an example circuit with five inputs and one output. Each gate is labelled with the input to output delay value associated with it. The two inputs 00000 and 11000 are shown in Figure 1.3 which cause a transition at output Y from 0 to 1 . Considering all possible input state pairs, the critical path $\mathrm{P}$ having 


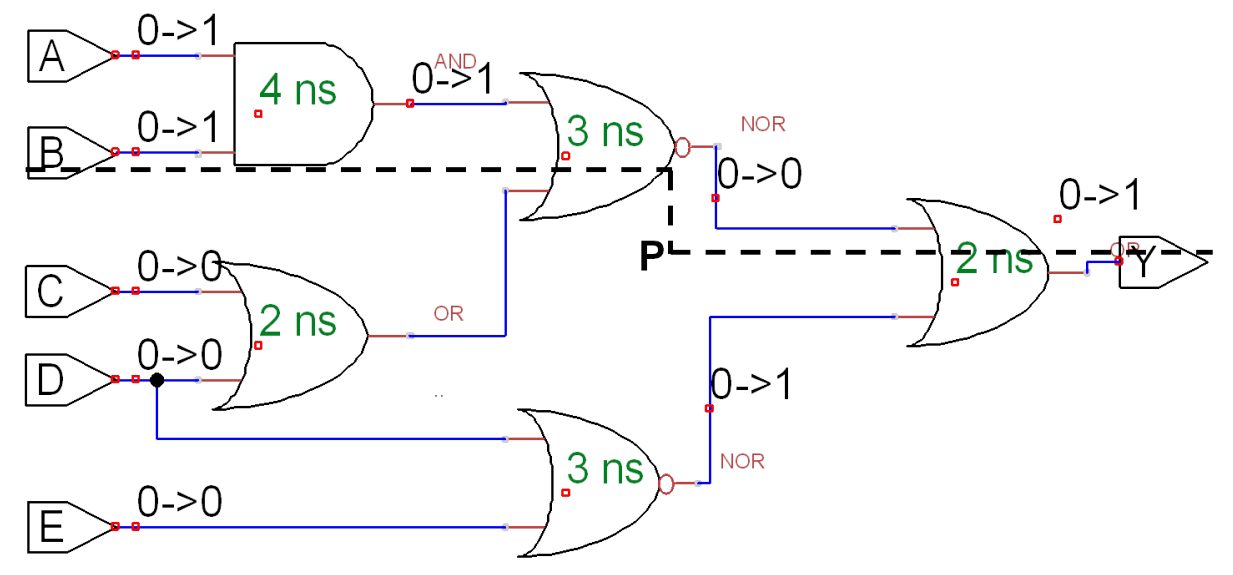

Figure 1.3: An example circuit showing the critical path $\mathrm{P}$.

the highest delay is selected and the delay is checked to be within a desired clock period. The critical path $\mathrm{P}$ is shown using a dotted line. The path delay of path P should be less than the desired clock period.

\subsubsection{MinVdd test}

For a given operating frequency, minVdd is the minimum supply voltage at which the circuit operates correctly [8]. The state of the circuit under test is defined by input binary values which excite the slowest speed paths. A set of values of minVdd voltages is obtained for different input frequencies. Parts having abnormal minVdd values are tagged as fails.

\subsection{Figures of Merit}

A part is manufactured, tested and used. Figure 1.4 shows the key figures of merit of an I.C test flow. The key figures of merit are yield loss, overkill and test escapes. Test escapes are the parts bad in use but pass the test. On the other hand, some parts good in use get rejected at a test and are called overkills. The yield loss is 


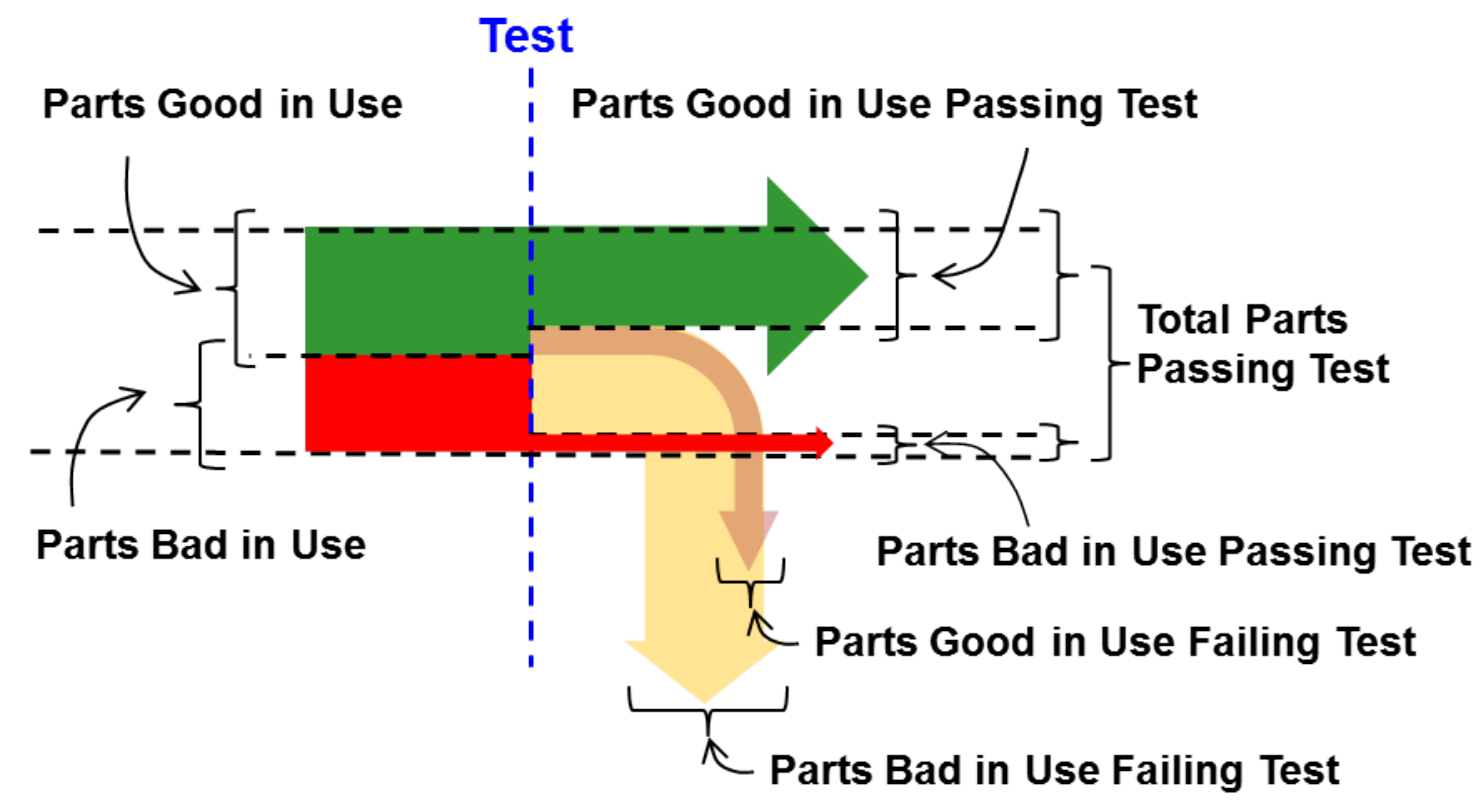

Figure 1.4: Figures of merit.

calculated as:

$$
\text { Yield loss }=\frac{\text { Total Parts Failing Test }}{\text { Parts Good in Use }+ \text { Parts Good in Use }}
$$

The overkills and test escapes are calculated as follows:

$$
\begin{gathered}
\text { Overkill }=\frac{\text { Parts Good in Use Failing Test }}{\text { Parts Good in Use }} \\
\text { Test Escape }=\frac{\text { Parts Bad in Use Passing Test }}{\text { Total Parts Passing Test }}
\end{gathered}
$$

An alternative to represent the overkill is the kill ratio [9]. The kill ratio calculates the yield impact rather than calculating the overkilled parts. Kill ratio calculates 
the healthy parts killed for each faulty part killed by test.

$$
\text { Kill Ratio, KR }=\frac{\text { Parts Bad in Use Failing Test }}{\text { Parts Good in Use Failing Test }}
$$

The test escapes expressed per million parts is DPPM (defective parts per million):

$$
\mathrm{DPPM}=\frac{\text { Parts Bad in Use Passing Test }}{\text { Total Parts Passing Test }} \times 1,000,000
$$

\subsection{Thesis Motivation}

Figure 1.5 shows the variation of values of an example state dependent parametric test for dies within a wafer and within a lot. The $\mathrm{x}$-axis shows the range of parametric test values for each of the four input states of the parametric test. The $\mathrm{y}$-axis represents the test value variation on six wafers and variation on a lot. Each horizontal bar represents the process variation in test values of an input state across all dies on the wafer or lot. A dot on the each of the horizontal bars shows the test value of a die picked from a wafer. The vertical dotted line represents the /fail test limits. The pass/fail test limit is set for each state. Dies having test values greater than the limit are rejected. Figure 1.5 shows two examples of non defective dies. The non defective die having state test values less than limits is tagged as pass. The non defective die, however, having test values greater than limits is rejected. For both non defective dies, there is little overlap between consecutive state test values. So, non-defective dies have a "natural" ordering of states by parametric test values. For example, a non defective die shown in figure will have an order:

$$
\text { State } 1<\text { State } 2<\text { State } 3<\text { State } 4
$$




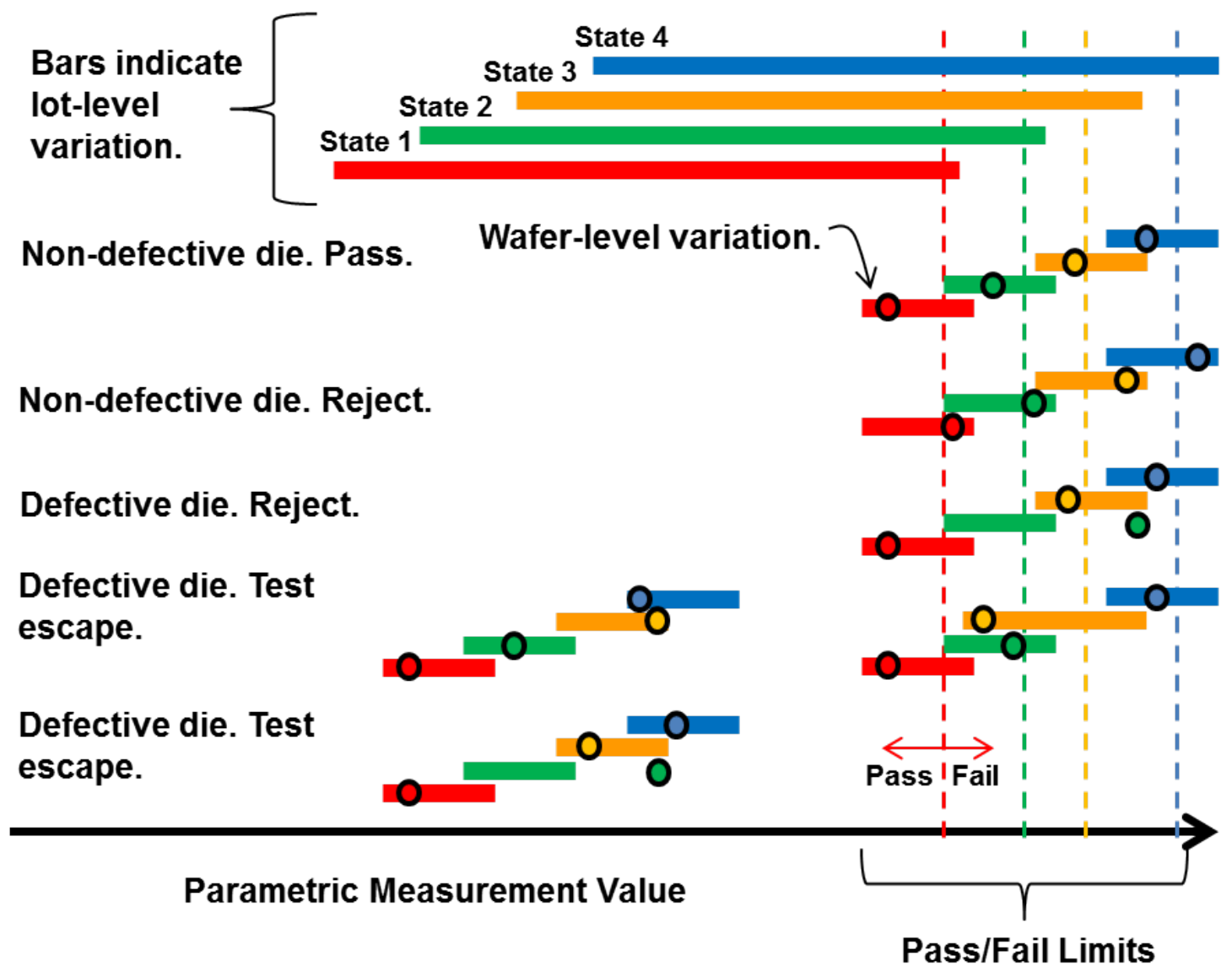

Figure 1.5: Illustration showing the parametric variation taking place within a wafer and a lot for four input states. Bars represent process variation of state dependent parametric test values. Dot represents the test value of a die for each of the four states. 
Process variation will cause some non-defective dies to violate test limits, and be rejected. A non-defective die has a "natural" ordering of states by the parametric

measurements. A defect in a die disrupts the natural ordering of states more severely than process variation. A high degree of order disruption indicates a defective die that should be killed. For example, any order disruption indicates a defective die. Even order disruption within process variation indicates a defective die. Process variation may disrupt the natural ordering of states, but the effect is small. For example, process variation causes no order disruption.

If a die is in lower end of process variation, the disruption in the test value will be less than test limits. The test would not be able to detect the defective die. This is shown by three examples of defective dies in Figure 1.5 which have defective test values less than limits.

This thesis motivates the need of intelligent selection of a single threshold using state orders instead of multiple test limits on each state which will reduce the test escapes while minimizing the overkills.

\subsection{Thesis Contributions}

This thesis demonstrates for the first time the use of the inter-die rank statistics to screen the test escapes. The statistical screen utilizes the inter-die ranks of a state dependent parametric test to differentiate between healthy and faulty dies. The statistical screen hypothesizes that healthy die test data have consistent state ranking. The screen, however, does not depend upon a prior knowledge of healthy die test data ranking. The benefit of the statistical screen is that it can be applied to any state-dependent test such as minVdd, delay, IDDQ and Fmax. A correlation 
mixture model is developed to model the tau distribution obtained from die pairs. The modified Estimation Maximization (EM) algorithm is used to differentiate the healthy and faulty components from the tau mixture. The probability of each die to be healthy or faulty is estimated. A single probability threshold is decided to separate the faulty dies from healthy dies.

\subsection{Thesis Organization}

The thesis is organized in four chapters. The background concepts are explained

in the Chapter 2. Chapter 2 also explains the previous work done by authors in the field of statistical post processing of parametric data. The correlation model proposed in this thesis is explained in Chapter 3. Chapter 4 contains the simulation results on synthetic data and production data. The conclusion and future work is explained in Chapter 5. 


\section{Chapter 2}

\section{Background Concepts and Previous work}

\subsection{Rank Order}

Rank order refers to the data transformation in which numerical values are replaced by their rank when the data is sorted from minimum to maximum [10]. For example, if $\mathrm{X}$ is a set of five measurements.

$$
\begin{gathered}
\text { 1. } X=\{21,45,32,65,36\} \\
\text { Rank-order }(X)=\{1,3,5,2,4\}
\end{gathered}
$$

The rank-order is insensitive to any monotonic transformation in the data. For example, increasing the value of each measurement in $\mathrm{X}$ by 2 , does not have any effect on rank-order.

$$
\begin{gathered}
X^{\prime}=X+2 \\
\text { 2. } X^{\prime}=\{23,47,35,67,38\} \\
\text { Rank-order }\left(X^{\prime}\right)=\{1,3,5,2,4\}
\end{gathered}
$$

Rank statistics is also called ordinal invariant statistics because the rank-order is independent of any type of monotonic variation in raw data. 


\subsection{Correlation}

The concept of statistical correlation is central to this thesis. Suppose a pair of random variables $\mathrm{X}$ and $\mathrm{Y}$ is sampled multiple times. If larger values of $\mathrm{Y}$ tend to be associated with larger values than $\mathrm{X}$, then $\mathrm{X}$ and $\mathrm{Y}$ are said to positively correlated. If larger values of $\mathrm{Y}$ tend to be associated with smaller values of $\mathrm{X}$, then $\mathrm{X}$ and $\mathrm{Y}$ are said to be negatively correlated. If there is no tendency for $\mathrm{Y}$ values to be associated with the $\mathrm{X}$ values, $\mathrm{X}$ and $\mathrm{Y}$ are said to be uncorrelated.

\subsection{Kendall's Tau Correlation}

The Kendall's rank correlation coefficient is a well known measure of correlation between two variables using the ranks of samples in the variable [11]. The Kendall's rank correlation coefficient is commonly referred to as tau and denoted by $\tau$. Let $\mathrm{X}=X_{1}, X_{2}, \ldots, X_{s}$ and $\mathrm{Y}=Y_{1}, Y_{2}, \ldots, Y_{s}$ be two set of $s$ measurements. A pair of measurements $\left(X_{i}, Y_{i}\right)$ and $\left(X_{j}, Y_{j}\right)$ is concordant, if

$$
\left(X_{i}-X_{j}\right)\left(Y_{i}-Y_{j}\right)>0
$$

A pair of measurements $\left(X_{i}, Y_{i}\right)$ and $\left(X_{j}, Y_{j}\right)$ is discordant, if

$$
\left(X_{i}-X_{j}\right)\left(Y_{i}-Y_{j}\right)<0
$$

The number of unique pairs between $s$ measurements is $s(s-1) / 2$. If $s_{c}$ and $s_{d}$ represents the number of concordant and discordant pairs respectively, tau between 
two data sets $\mathrm{X}$ and $\mathrm{Y}$ of each sample size $s$ is calculated as:

$$
\tau=\frac{2\left(s_{c}-s_{d}\right)}{s(s-1)}
$$

The range of tau is from -1 to 1 .

A measurement pair is tied if $X_{i}=X_{j}$ or $Y_{i}=Y_{j}$. In the case of tied measurements, total number of unique pairs get reduced due to the ties. The tau calculated with ties is:

$$
\tau=\frac{s_{c}-s_{d}}{\sqrt{\left(\frac{s(s-1)}{2}-\frac{\sum\left(s_{x}\left(s_{x}-1\right)\right)}{2}\right)\left(\frac{s(s-1)}{2}-\frac{\sum\left(s_{y}\left(s_{y}-1\right)\right)}{2}\right)}}
$$

where $s_{x}$ and $s_{y}$ are number of tied samples in data sets $\mathrm{X}$ and $\mathrm{Y}$ respectively.

\subsubsection{Graphical Representation of Tau}

Figure 2.1 shows the discordance plot comparing the ranked parametric test states measured on two example dies 1 and 2. For each die, the test values are measured using eight different input states. The states are rank ordered according to the test values sorted from minimum to maximum. The ranked state order is plotted for die 1 and die 2. The same states of both the dies are connected using the dotted lines. The dotted lines shows the unchanged rank of the input states 4 and 8 for

die 1 and die 2. The dotted lines also show the forward and backward shifting of the ranks of the input states 1, 2, 3, 5, 6 and 7 for die 1 and die 2. The numbers of crossings $c$ in the Figure 2.1 represent the number of discordant pairs $s_{d}$ of two measurements. If $s$ is the number of states, there will be $s(s-1) / 2$ unique state 


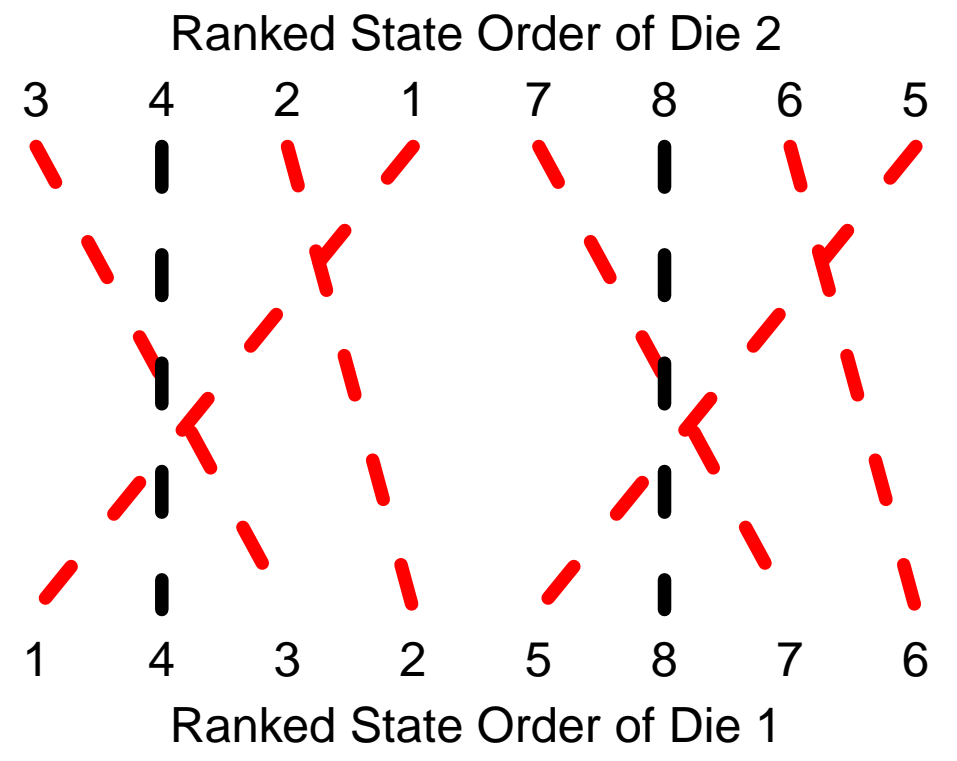

Figure 2.1: Discordance plot showing correlation between two measurements. The labels are the state numbers.

pairs. The tau is calculated using the discordance plot as [12]:

$$
\tau=1-\frac{4 s_{d}}{s(s-1)}
$$

Equation 2.10 is same as Equation 2.8.

In Figure 2.1, the number of crossings in the discordance plot is equal to 8. The tau is equal to:

$$
\begin{aligned}
\tau & =1-\frac{4 \times 8}{8(8-1)} \\
& =1-\frac{32}{56} \\
& =0.43
\end{aligned}
$$




\subsection{IDDQ Current Signature}

Figure 2.2 shows the difference between IDDQ measurements of a defect free circuit and a defective circuit. The first column labelled as "Circuit" shows three types of 3-input AOI (and-or-invert) gate circuits: a defect free AOI circuit, an AOI circuit with a resistive short between Vdd and output node and an AOI circuit with a resistive short between Vdd and ground. The second column labelled as

"IDDQ signature" shows IDDQ values of the three circuits for each of the eight possible input states. In Figure 2.2a, no input combination is able to activate a static path between Vdd and ground. The IDDQ flowing from Vdd to ground is the background leakage current of transistors.

Figure $2.2 \mathrm{~b}$ shows the AOI circuit with a resistive short $R_{d}$ between output node and Vdd. The input states $\mathrm{ABC}=000,001,010$ do not activate any static path between Vdd and ground. The circuit conducts three levels of defect-indicating IDDQ for three sets of input states: first when $A B C=011$, second higher level of IDDQ when $\mathrm{ABC}=100,101,110$ and a third higher level of IDDQ when $\mathrm{ABC}=111$. In Figure 2.3c, the short $R_{d}$ is directly between $\mathrm{Vdd}$ and ground. A constant high IDDQ flows from Vdd to ground irrespective of any input combination.

Figure 2.2 shows that a defect can cause an IDDQ signature for all possible input states. Figure $2.2 \mathrm{~b}$ shows a multilevel IDDQ signature while $2.2 \mathrm{c}$ shows an elevated IDDQ signature. A resistive short occurring between a switching node and any other node is called an active defect. Only some of the input states activate the static IDDQ flow from Vdd to ground through active defect. A short between Vdd 


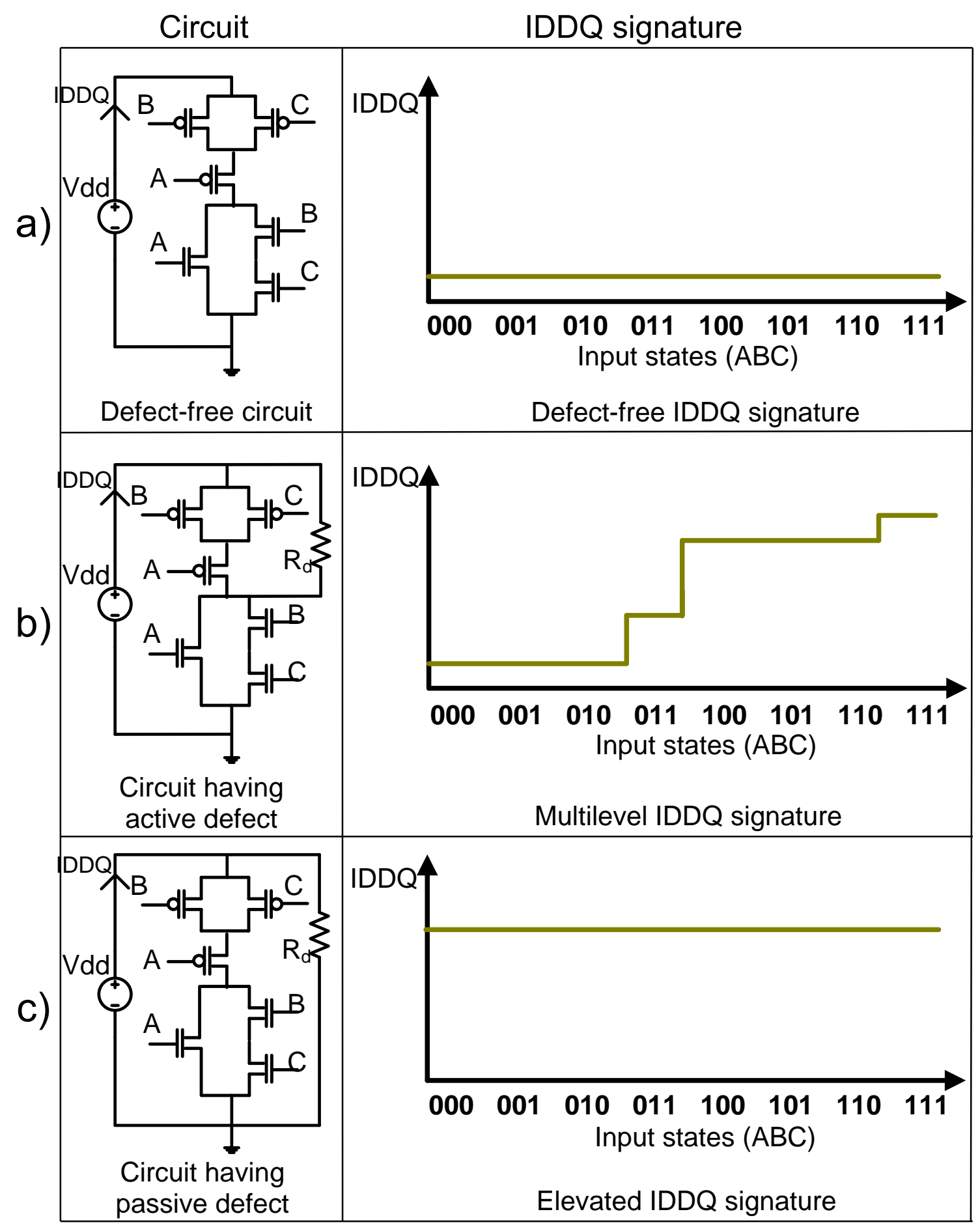

Figure 2.2: Difference between IDDQ for input states of a defect-free circuit and a defective circuit. 
and ground will always cause an elevated static IDDQ flow despite any change in input state combination. The defect is called a passive defect.

Figure 2.3 and 2.4 show IDDQ plots of dies from real 90nm production wafer-sort data which will be analyzed in this thesis. For each die, IDDQ is measured on 64 input states. In 2.3a and 2.4a, the x-axis represents the 64 input states in application order while in $2.3 \mathrm{~b}$ and $2.4 \mathrm{~b}$, the $\mathrm{x}$-axis represents the 64 input states in sorted IDDQ values. The y-axis represents the range of IDDQ values for all 64 states from minimum IDDQ to maximum IDDQ measured on that particular die. Figure 2.3 shows the example of a defect-free die. The IDDQ values in a defect free die across all input states are consistent. Figure 2.4 shows a IDDQ plot of a defective die. The elevated states cause the defective IDDQ to activate showing a two level current signature.

\subsection{Previous Work}

Deep sub-micron technologies have large intrinsic leakage current which gets mixed with elevated defect current. It becomes difficult to detect current due to defects which give a weak signal [13]. Research efforts have focused on the statistical post-processing (SPP) of test data to screen defective devices [14. Statistical post-processing is becoming a significant tool for identifying potential failures. Statistical post-processing on IDDQ test data is a widely discussed topic in the published literature largely due to high defect coverage of IDDQ.

One of the earliest papers describing IDDQ current signatures has been published by Gattiker and Maly [6]. The authors characterize the defects as active and passive defects. The active defect results in a multilevel current signature. Outlier 

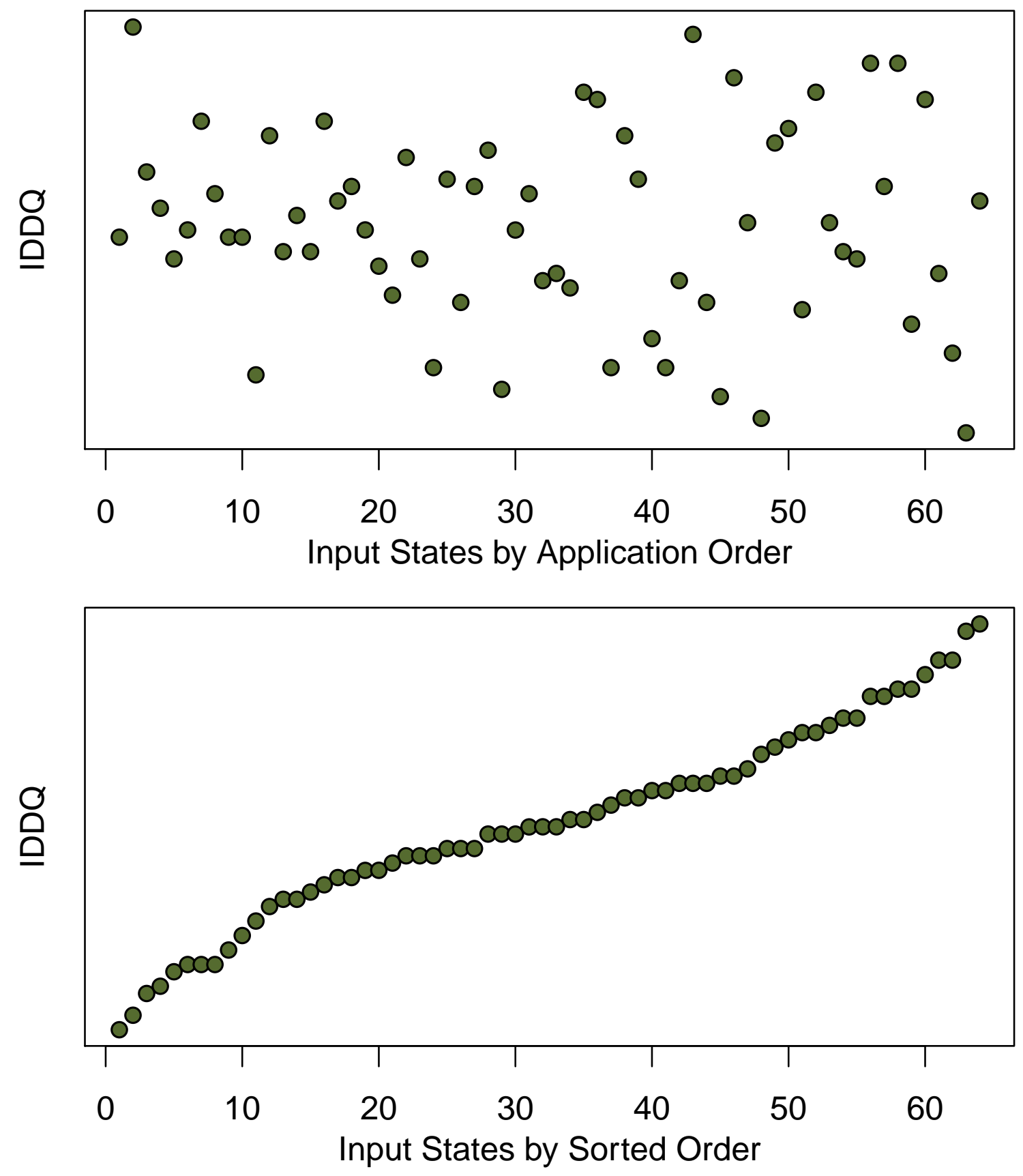

Figure 2.3: IDDQ plots of a defect free die. 

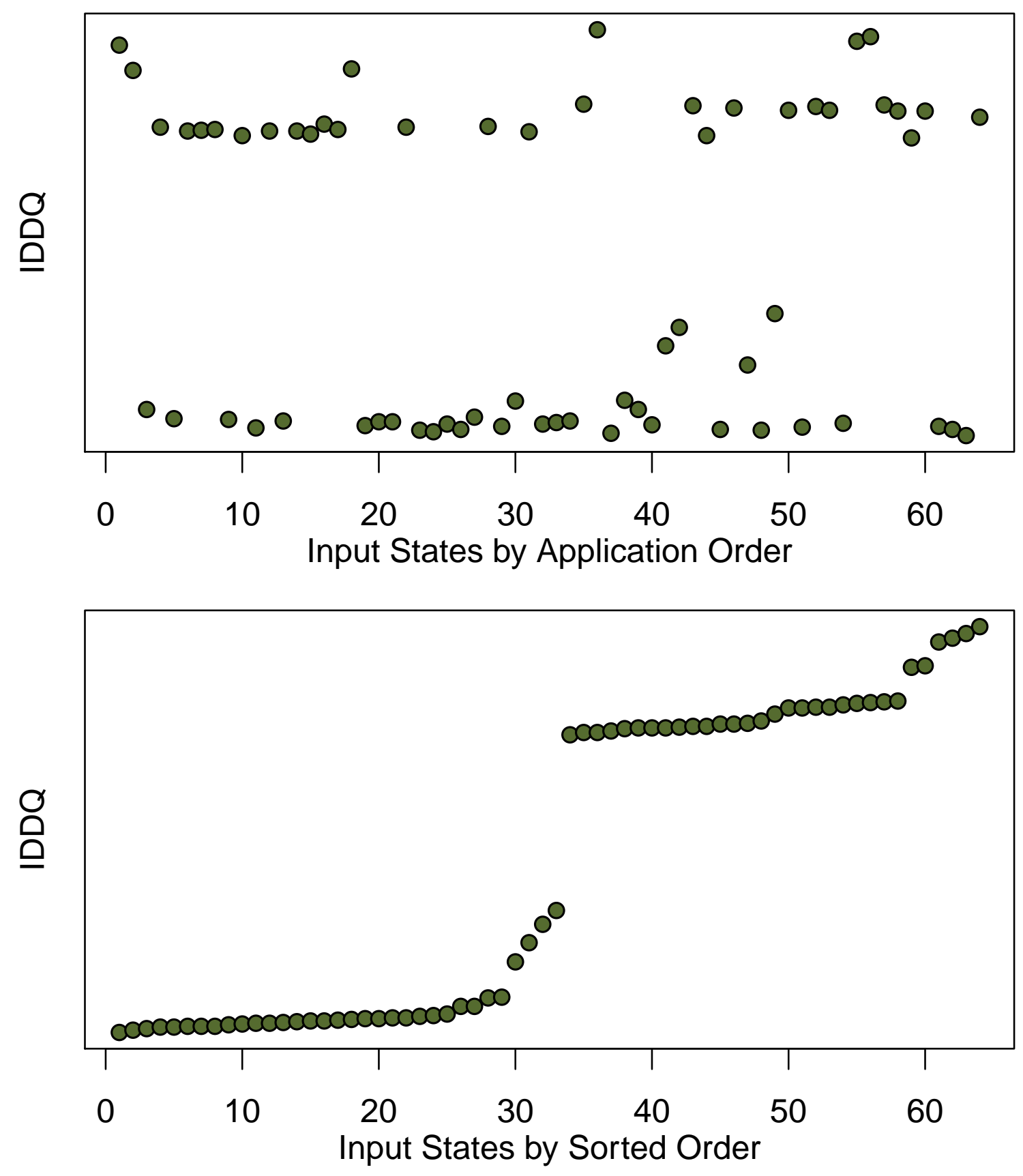

Figure 2.4: IDDQ plots of a defective die. 
dies are selected using steps in the IDDQ signature. The input states are identified which activate the step in current signature on the leakage paths. Die to die IDDQ variation is removed from measurements by identifying the circuit personality current signature. Dies having step signatures even after removal of variations are declared as fails.

Thibeault proposed the $\triangle \mathrm{IDDQ}$ method which attempted to remove the process variations taking place from die to die [15]. For an input state pair(i,i-1), the $\triangle \mathrm{IDDQ}$ can be represented as:

$$
\Delta \mathrm{IDDQ}=\operatorname{IDDQ}(\mathrm{i})-\operatorname{IDDQ}(\mathrm{i}-1)
$$

Since the IDDQ contains die to die process variations, the author proposed that the $\triangle \mathrm{IDDQ}$ removes the variation by subtracting out the process variance in both the input states. Assuming IDDQ state-to-state variance and IDDQ die-to-die variance as independent, the variance of the IDDQ distribution, $\sigma_{i d d q}^{2}$ is proposed as sum of the state to state variance, $\sigma_{\text {state }}^{2}$ and die-to-die variance, $\sigma_{i c w}^{2}$. The variance of $\triangle \mathrm{IDDQ}$ distribution, $\sigma_{\text {delta }}^{2}$ is expected to be twice the state to state variance, $\sigma_{\text {state }}^{2}$.

$$
\begin{gathered}
\sigma_{\text {iddq }}^{2}=\sigma_{\text {state }}^{2}+\sigma_{\text {icw }}^{2} \\
\sigma_{\text {delta }}^{2}=2 \times \sigma_{\text {state }}^{2}
\end{gathered}
$$

Equations 2.15 and 2.16 are experimentally verified on Sematech data. A probabilistic framework is developed which reduces the probability of false fails decisions as compared to the standard IDDQ method. A minimum number of test states 
required to obtain a high test quality is also calculated using the probability framework.

Statistical post-processing using IDDQ Intra-die correlation values has been proposed by Gudavalli and Daasch in [16]. The Intra-die correlation is calculated using two measurements of IDDQ at two different temperatures on a die. When Intra-die correlation is found to be low then the die is tagged as defective. The confidence bounds of the correlation distribution of IDDQ are obtained using Monte Carlo simulations. Dies which are outside the confidence intervals are tagged as outliers. The Intra-die screen is verified on the production wafers.

This thesis is an extension of work done by Gudavalli and Daasch [16]. The correlation between test responses of die pairs is utilized to identify the defective dies based on the concepts of current signatures. This thesis attempts to reduce the effects of process variation by calculating rank-order correlation of input states of parametric test data of die pairs. 


\section{Chapter 3}

\section{Defect Detection Model}

\subsection{Kendall's Tau Calculation}

Each die has a set of state-dependent parametric measurements. The measurements can be ranked and each measurement is labelled with it's rank. Tau can be computed for each pair of dies on a wafer using the list of ranks for each die.

Figure 3.1 shows the tau matrix for inter-die pairs on an example wafer. The example wafer has $n$ dies. Each cell in the matrix has tau for a die pair. The subscript associated with each tau value in the box represents the die pair, i.e, $\tau_{i j}$ is calculated from die $i$ and die $j$ pair. The diagonal values show the correlation between same die pairs which will be always 1 .

$$
\tau_{i j}=1 \quad \text { for }(i=j)
$$

In Figure 3.1, for each tau value in lower triangle of tau matrix, there is equal and corresponding value in upper triangle of tau matrix shown in same color in the matrix. The lower triangle has all the possible combinations of inter-die tau values.

$$
\tau_{i j}=\tau_{i j} \quad \text { for }(i \neq j)
$$




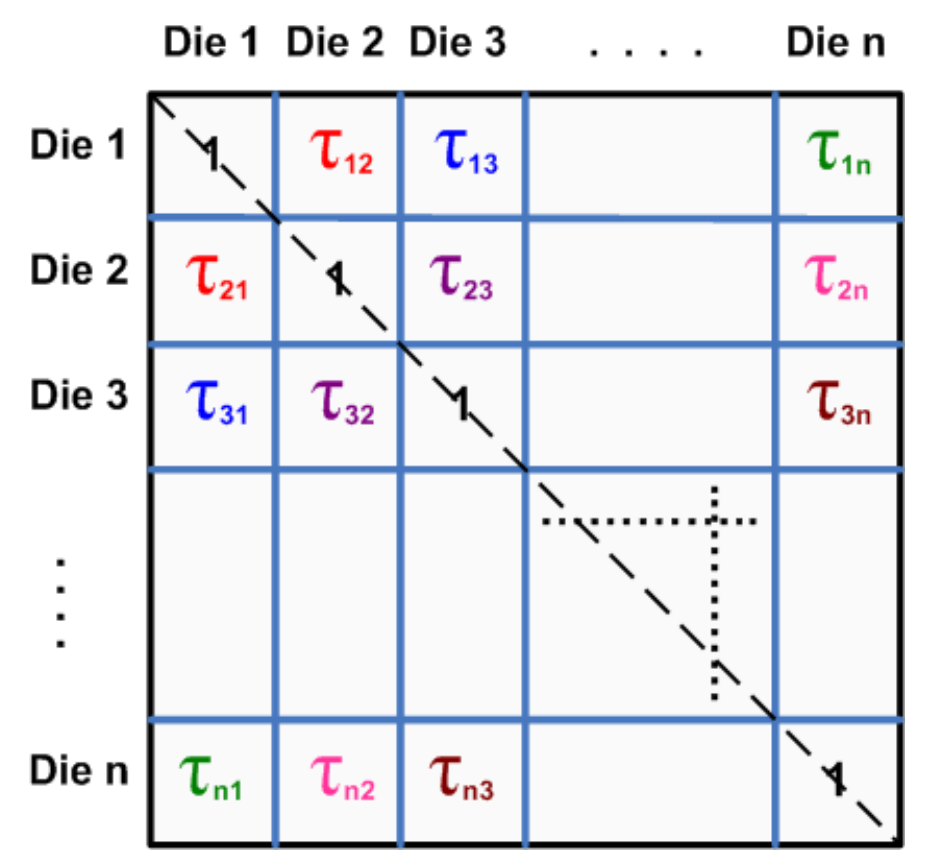

Figure 3.1: Tau matrix of die pairs.

\subsection{Correlation Model}

This thesis presents a correlation model for inter-die tau distribution on a wafer. Figure 3.2 shows the graphical representation of the correlation model. The correlation model hypothesizes that tau distribution calculated from all the die pairs on a wafer is a mixture of two distributions. One distribution is called healthy component and the other distribution is called faulty component. The healthy component comes from the pairing of the healthy-healthy dies. The faulty component comes from pairing of healthy-faulty, faulty-healthy and faulty-faulty dies on a wafer.

A healthy die is hypothesized to have a single particular rank order of parametric test responses which is not known a priori. A faulty die will have different rank orders of test responses. The tau calculated between healthy-healthy die pairs 
will be high as compared to tau calculated between healthy-faulty die pairs. The distribution for healthy-faulty die pairs is exactly identical to faulty-healthy die pairs because of the symmetry of the tau matrix as shown in Figure 3.1. Due to process variations, a defect can affect the faulty die test responses in random ways. So, the tau calculated between faulty-faulty die pairs can have a broad range of tau.

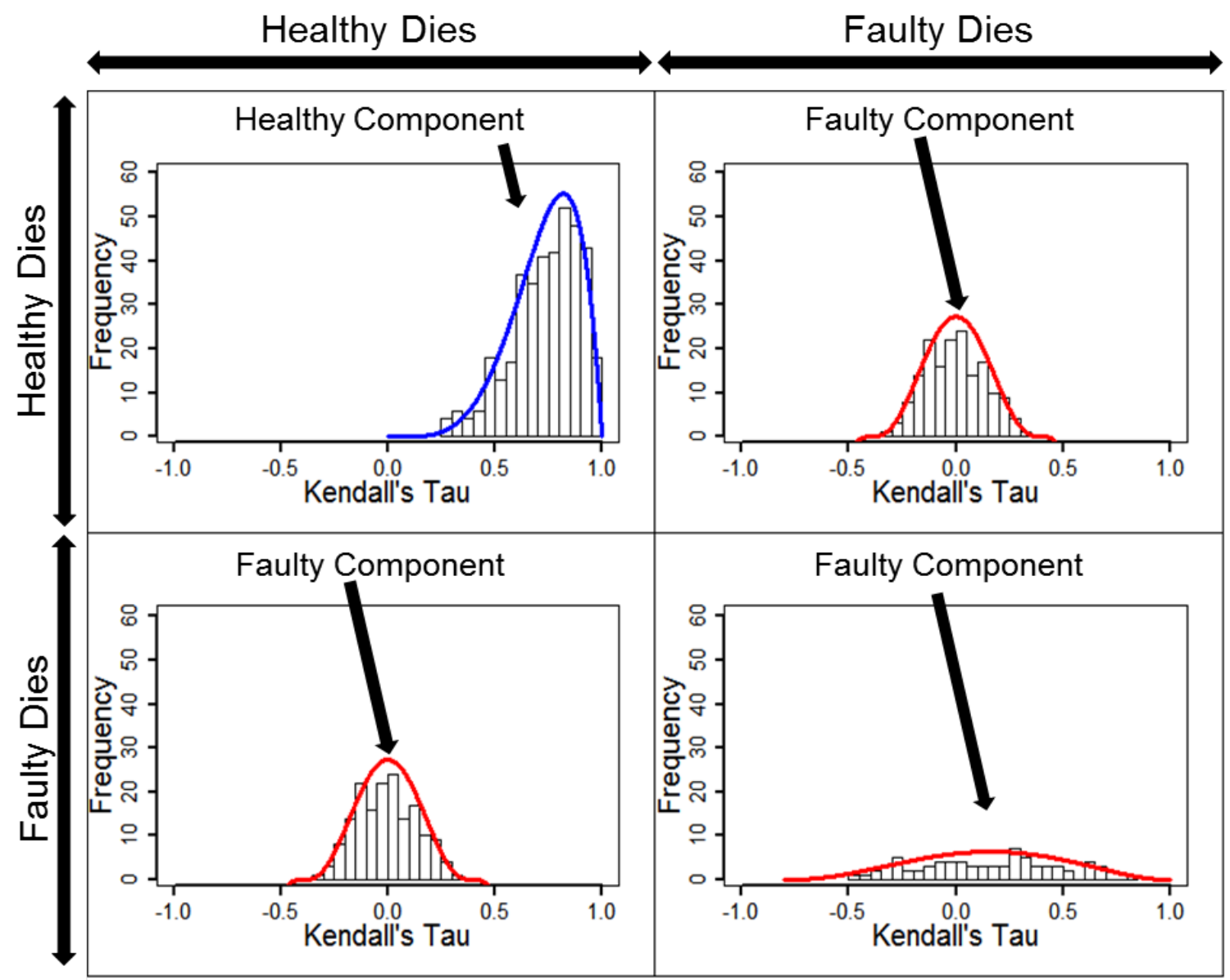

Figure 3.2: Correlation model showing inter-die tau distributions calculated using die pairs. 
The proportion of healthy-healthy, healthy-faulty, faulty-healthy and healthy-healthy pairs depend upon the yield of the wafer. For high yielding wafers, the number of tau samples for healthy-healthy die pairs will be larger while smaller for all other die pairs. The faulty-faulty component will be ignored because the sample size of tau from faulty-faulty die pairs for high yielding wafers is low.

Let $y$ represent the yield of the wafer having $n$ number of dies. The number of healthy dies will be $y \times n$. The number of faulty dies is $(1-y) \times n$. For $n$ dies, total number of possible pairs is $n^{2}$. If all the same die pairs tau $(\tau=1)$ are ignored, the number of tau samples for different die pairs are as follows:

$$
\begin{gathered}
\text { healthy-healthy tau samples }=y n(y n-1) \\
\text { healthy-faulty tau samples }=y(1-y) n^{2} \\
\text { faulty-faulty tau samples }=(1-y) n[(1-y) n-1]
\end{gathered}
$$

The number of tau samples in healthy component is equal to number of tau samples in healthy-healthy tau distribution.

$$
\text { Healthy component tau samples }=y n(y n-1)
$$

The number of tau samples in faulty component is equal to sum of tau samples in healthy-faulty tau distribution, faulty-healthy tau distribution and faulty-faulty distribution.

$$
\text { Faulty component tau samples }=2 y(1-y) n^{2}+(1-y) n[(1-y) n-1]
$$


Figure 3.3 shows the mixture of synthesized healthy-healthy, healthy-faulty, faultyhealthy and faulty-faulty distributions of the proposed correlation model. The rightmost density curve (blue curve) on the mixture model shows the healthy component density while the leftmost density curve (red curve) shows the faulty component density.

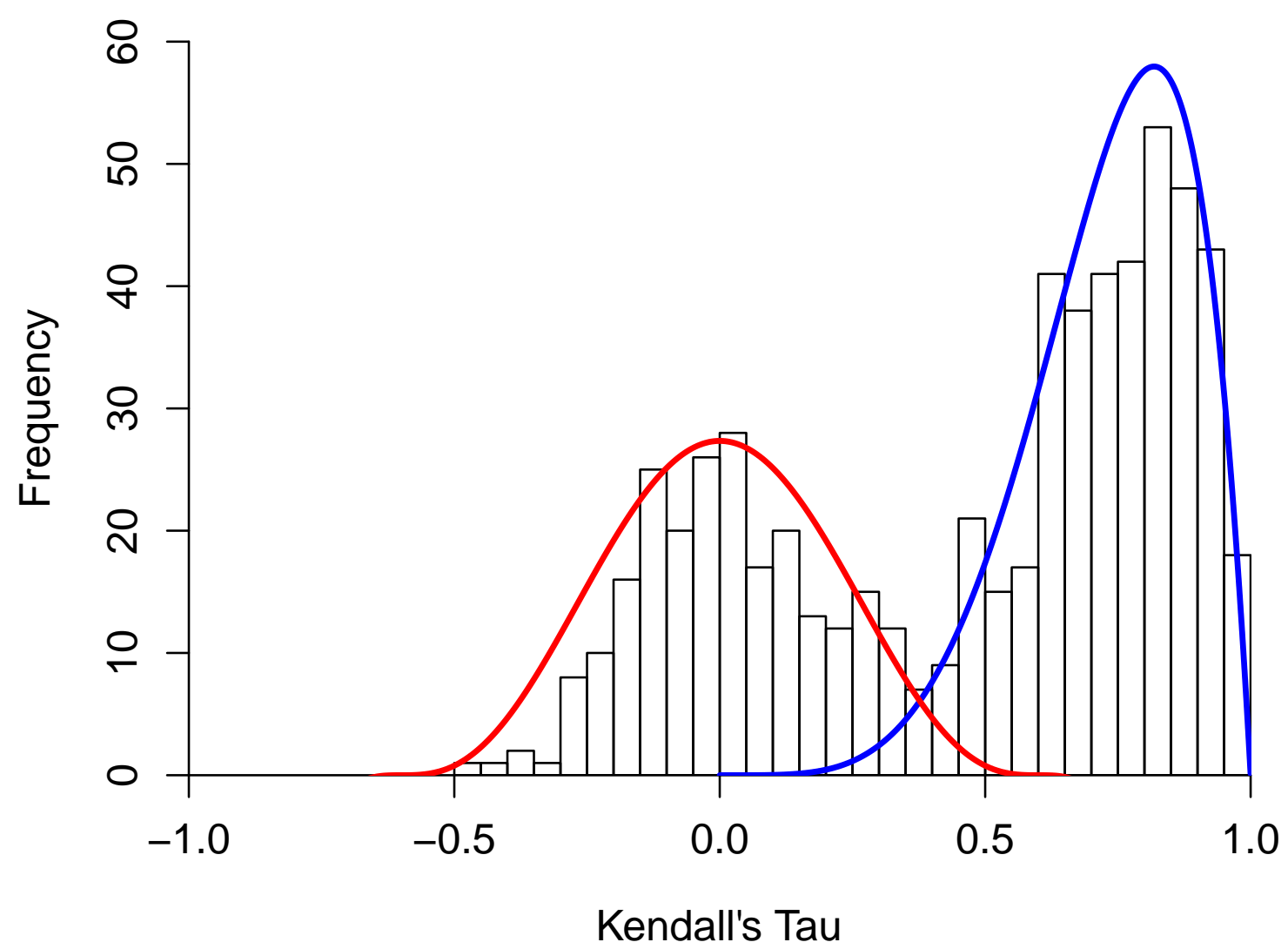

Figure 3.3: Correlation model mixture of synthesized healthy-healthy, healthyfaulty and faulty-healthy distributions. The healthy-healthy distribution is shown by rightmost red curve while the healthy-faulty and faulty-healthy distributions is shown by leftmost blue curve.

Figure 3.4 shows a tau distribution calculated from a wafer in a $90 \mathrm{~nm}$ production dataset which will be analyzed in this thesis. The tau is calculated for each die 
pair on the wafer by comparing the rank-order of 64 IDDQ test values of each die.

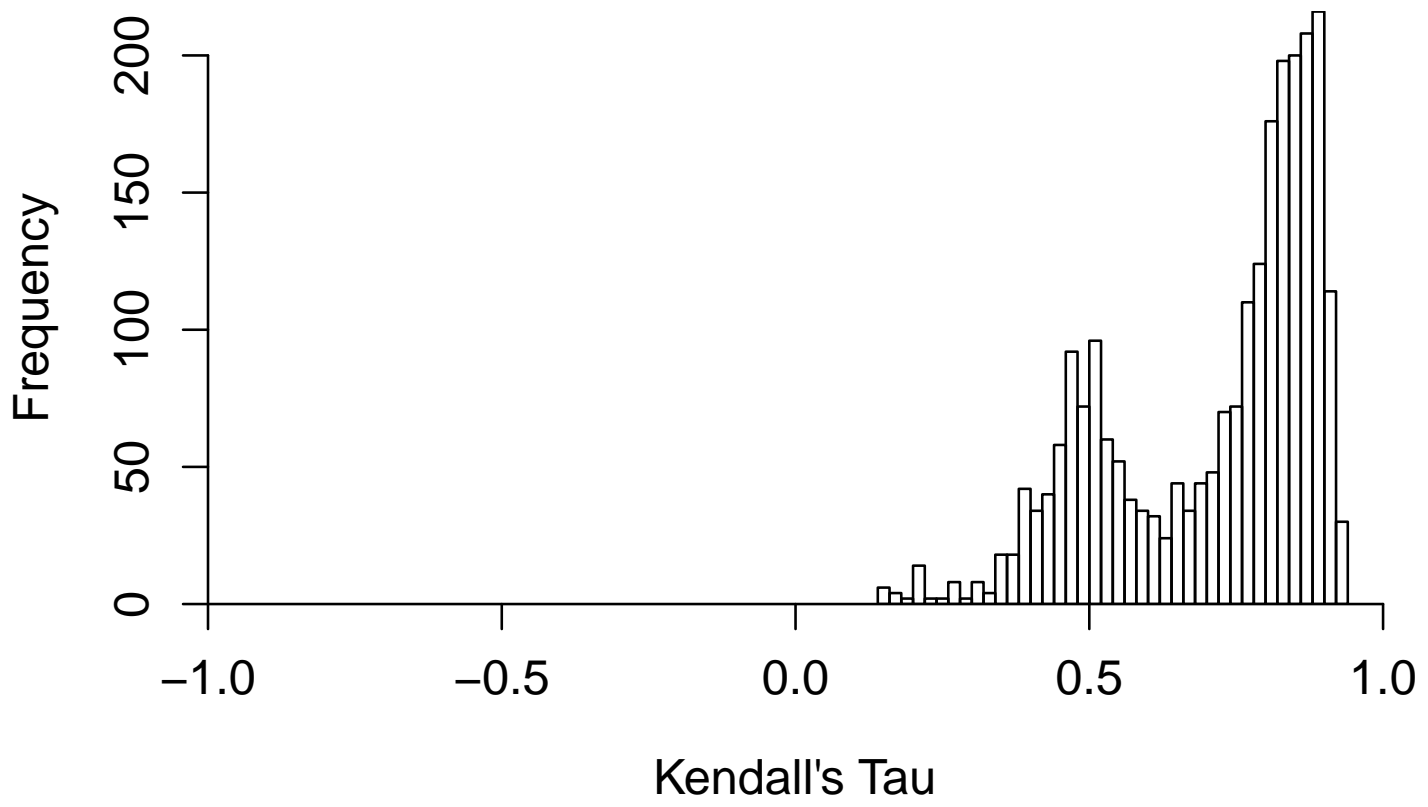

Figure 3.4: Tau mixture histogram calculated from a wafer of the production 90nm dataset.

\subsection{Correlation Mixture Model Building}

The tau distribution is a finite distribution between -1 and 1 . The proposed correlation mixture can be modelled by superimposing two generalized beta distributions. The standard beta distribution covers the interval $[0,1]$. A generalized beta distribution scales the standard beta distribution to cover any finite interval $[l, h][17]$.

A generalized beta distribution is a probability distribution defined between finite intervals $l$ and $h$ and defined by two positive shape parameters $\alpha$ and $\beta$. The 
generalized beta distribution gives the probability density of a value $\mathrm{x}$ on the interval $[l, h]$ :

$$
\operatorname{Beta}(\mathrm{x} \mid \alpha, \beta)=\frac{(x-l)^{\alpha-1}(h-x)^{\beta-1}}{B(\alpha, \beta)(h-l)^{\alpha+\beta-1}}
$$

where $B$ is the beta function

$$
B(\alpha, \beta)=\int_{0}^{1} t^{\alpha-1}(1-t)^{\beta-1} \mathrm{~d} t
$$

The two component generalized beta mixture will model the proposed correlation mixture using two probability distributions. One probability distribution represents the healthy component obtained from healthy die pair responses. The second probability distribution represents the faulty component obtained from die pairs involving faulty responses. The tau mixture distribution can be represented as:

$$
f\left(\tau \mid p, \alpha_{F}, \beta_{F}, \alpha_{H}, \beta_{H}\right)=p \operatorname{Beta}\left(\alpha_{F}, \beta_{F}\right)+(1-p) \operatorname{Beta}\left(\alpha_{H}, \beta_{H}\right)
$$

where $\alpha_{H}$ and $\beta_{H}$ are the shape parameters of the healthy component, $\alpha_{F}$ and $\beta_{F}$ are the shape parameters of the faulty component and $p$ is the mixing proportion probability of faulty component in the tau mixture. The mixing proportion probability of healthy component is $1-p$ in the tau mixture. The healthy mixing proportion is the squared yield of the wafer.

Let $\tau_{i j}$, denote the tau calculated from a die pair, where $n$ is the total number of dies on a wafer. The subscripts $i$ and $j$ represent the die number from which the tau is calculated. The maximum number of die pairs is equal to $n(n-1)$, if $i \neq j$. 
An indicator variable $z_{i j}$ is introduced for each tau $\tau_{i j}$ where

$$
z_{i j}=\left\{\begin{array}{cl}
1 & \text { if } \tau_{i j} \text { comes from faulty component } \\
0 & \text { if } \tau_{i j} \text { comes from healthy component }
\end{array}\right.
$$

The set of values of $z_{i j}$ and $\tau_{i j}$ represent the complete data. The mixing proportion probability of faulty component, $p$ can be calculated as:

$$
p=\frac{\sum_{\substack{i=1 \\ i \neq j}}^{n} \sum_{j=1}^{n} z_{i j}}{n(n-1)}
$$

\subsubsection{Estimation Maximization (EM) algorithm}

EM algorithm can be used to estimate the two component correlation mixture model parameters. EM algorithm is a well known statistical tool developed for problems involving mixture estimation. The EM is an iterative method which finds the maximum likelihood estimates (mle's) of the unknown parameters in a statistical model [18]. The EM iteration alternates between performing an expectation (E) step, which computes the expectation of the log-likelihood evaluated using the current estimate for the unknown parameters, and a maximization (M) step, which computes the unknown parameters maximizing the expected log-likelihood found on the E step.

Figure 3.5 illustrates the flow of the modified EM algorithm. The round boxes in flowchart represent the start and end of the modified EM algorithm. The triangle is the decision box. The correlation step shown in dashed line box modifies the 
original EM algorithm. The original EM treats each vector of $\left\{\tau_{i j}, z_{i j}\right\}$ as independent and identically distributed for the two components. The iid property of EM is modified as the tau values are associated with die pairs and cannot be treated as independent. The modified EM algorithm flow is described as follows:

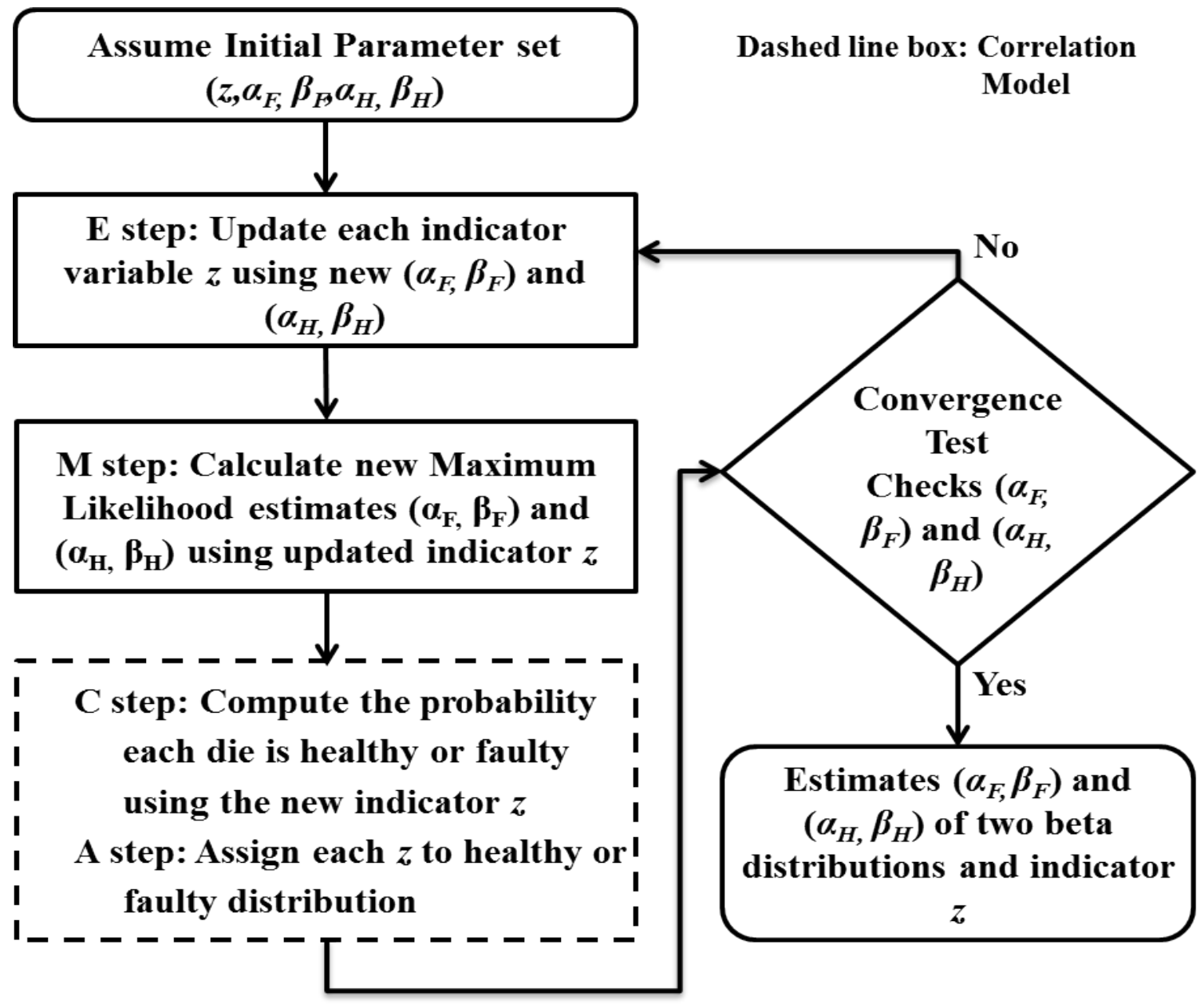

Figure 3.5: Flow chart of modified EM algorithm. The box in dashed lines shows the modified step in the EM algorithm.

1. Initialization step: The indicator variable $z_{i j}$ is initialized for each die pair. For the half of the $\tau$ population, $z_{i j}$ is initialized as 1 while the other half 
of $z_{i j}$ is initialized as 0 . Shape parameters $\alpha_{F}, \beta_{F}, \alpha_{H}, \beta_{H}$ are also initialized having values equal to 1.

2. Estimation step: The mixing proportion probability is calculated using Equation 3.12. Using the probability density function of a four parameter beta distribution, each $z_{i j}$ is updated.

$$
z_{i j}=p \operatorname{Beta}\left(\tau_{i j} \mid \alpha_{F}, \beta_{F}\right)+(1-p) \operatorname{Beta}\left(\tau_{i j} \mid \alpha_{H}, \beta_{H}\right)
$$

3. Maximization step: Using non linear maximization of log-likelihood function, the likelihood-maximizing shape parameters $\alpha_{F}, \beta_{F}, \alpha_{H}, \beta_{H}$ are calculated. The log-likelihood function is given by:

$$
\begin{aligned}
\log -\operatorname{likelihood}(\alpha, \beta, z)= & \sum_{\substack{i=1 \\
i \neq j}}^{n} \sum_{j=1}^{n}\left[z_{i j} \log \left\{\operatorname{Beta}\left(\tau_{i j} \mid \alpha_{F}, \beta_{F}\right)\right\}\right. \\
& \left.+\left(1-z_{i j}\right) \log \left\{\operatorname{Beta}\left(\tau_{i j} \mid \alpha_{H}, \beta_{H}\right)\right\}\right]
\end{aligned}
$$

Non linear maximization returns a log-likelihood value which is stored as a last-likelihood.

\section{Correlation step:}

4.1 Compute step: For each die, $z_{i j}$ values are normalized which gives a probability for each die $d_{i}$ being faulty.

$$
d_{i}=\frac{\sum_{\substack{j=1 \\ i \neq j}}^{n} z_{i j}}{n-1}
$$

The midpoint of die probabilities is chosen as threshold. If a die is 
above the threshold, the die is given a faulty probability equal to 1 and if below the threshold, the die is given a faulty probability equal to 0 .

4.2 Assignment step: Using the die faulty probability, $z_{i j}$ is updated according to correlation model. Considering die pairs, if both the dies are healthy, $z_{i j}$ is assigned the value 0 . If one or both of the dies in the pair are faulty, $z_{i j}$ is assigned the value 1 .

$$
\begin{gathered}
\operatorname{If}\left\{\left(d_{i}==0\right) \& \&\left(d_{j}==0\right)\right\} \\
\text { then } z_{i j}=0 \\
\text { else } z_{i j}=1
\end{gathered}
$$

5. Check Convergence step: With a new set of $z_{i j}$ and shape parameters $\alpha_{F}, \beta_{F}, \alpha_{H}, \beta_{H}$ estimation and maximization step is repeated. The maximization step gives a current likelihood value which is compared with lastlikelihood value. If the difference between the successive log-likelihoods is greater than 0.1 , the estimation and maximization step is repeated. If the difference is less than 0.1 or iteration count of EM is greater than 1000, the modified EM has converged.

The modified EM converges to the shape parameter estimates $\alpha_{F}, \beta_{F}, \alpha_{H}, \beta_{H}$ of the tau mixture and to the probability of each tau belonging to the faulty component. The total number of die pairs is $n(n-1)$, if $i \neq j$. Since, the healthy component mixing proportion is $(1-p)$, there are $(1-p) n(n-1)$ healthy component values. On the other hand, if $y$ is the yield of the wafer, then number of healthy component values $=y n(y n-1)$. 
Since mixing proportion probability of healthy component $(1-p)$ is known after modified EM converges, the approximate yield of the wafer can be solved as:

$$
y n(y n-1)=(1-p) n(n-1)
$$

Since $n \geq 1$, the Equation 3.16 can be approximated as:

$$
y^{2} n^{2}=(1-p) n^{2}
$$

The yield of the wafer is solved as:

$$
\text { Yield of wafer, } y \simeq \sqrt[2]{(1-p)}
$$

Using the probability of each tau $z_{i j}$, the probability of each die $d_{i}$ belonging to the faulty component is also computed using the Equation 3.15.

\subsection{Monte Carlo Simulations}

Monte Carlo is a statistical technique in which random samples are used to simulate data. Since the user knows the underlying model from which the simulated data is generated, statistical analysis such as modified EM method used in this thesis may be tested with a range of input data.

The modified EM algorithm is tested for results of shape parameters and the yield using Monte Carlo simulations before application of production data. For testing, two component beta mixture distributions are synthesized. By simulating different input conditions, sensitivity of modified EM to different mixture distributions is 
assessed.

The Monte Carlo testing of modified EM works in following steps:

1. The total number of dies on a wafer, yield of the wafer, shape parameters $\left(\alpha_{H}, \beta_{H}\right)$ and $\left(\alpha_{F}, \beta_{F}\right)$ of healthy and faulty component distributions respectively are given as inputs to the Monte Carlo simulator.

2. The simulator synthesizes a healthy beta component distribution using random beta samples from shape parameters $\left(\alpha_{H}, \alpha_{H}\right)$ in the interval $(-1,1)$. The simulator also synthesizes a faulty beta component distribution using random beta samples from shape parameters $\left(\alpha_{F}, \alpha_{F}\right)$ in the interval $(-1,1)$.

3. The two component beta mixture is given to the modified EM method. The modified EM method estimates the healthy and faulty component distribution shape parameters and the probability of each tau belonging to either healthy or faulty component distribution.

4. The die faulty probability is calculated using the tau faulty probability. A probability threshold is chosen above which all the dies are declared as faulty and below which all the dies are declared as healthy.

5. The estimated yield and shape parameters are compared with user given yield and the shape parameters.

Figure 3.6 shows the two component beta mixture generator synthesizing a mixture of two beta distributions. The whole box represents a wafer having $n$ number of dies. The four sub blocks in Figure 3.2 are filled by the two synthesized beta distributions using the proportion of tau samples mentioned in Equations 3.3, 
3.4 and 3.5. In Figure 3.6, the bold tau values represent the healthy component distribution in the mixture indicating tau values coming from healthy-healthy die pairs. The underlined tau values represent the faulty component distribution in the mixture indicating tau values coming from healthy-faulty, faulty-healthy and faulty-faulty pairs.

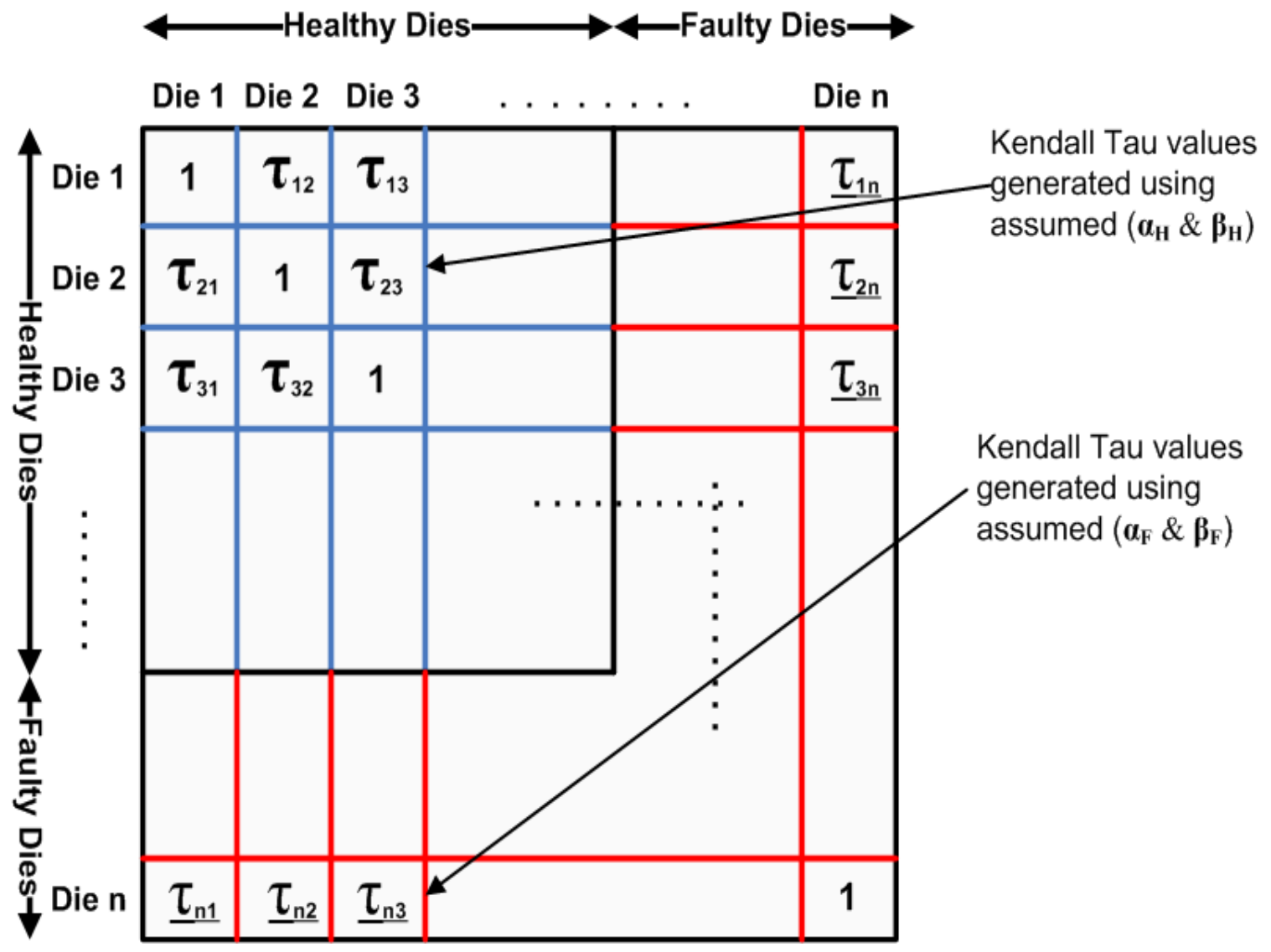

Figure 3.6: Two component synthetic beta mixture generator. Bold tau values are healthy component tau values while underlines tau values are faulty component tau values.

To check the sensitivity of modified EM to uncertainty in inputs, the following testing scenarios are used: 
1. Separation between two input component distributions: By changing the shape parameters $\alpha_{F}, \beta_{F}, \alpha_{H}, \beta_{H}$, the two input component distributions in the mixture model can either be seen as widely separated as shown in Figure 3.7a or mixed which looks like a single distribution as in Figure 3.7b. Figure $3.7 \mathrm{a}$ and $\mathrm{b}$ is simulated using 100 dies and a yield of $75 \%$. The rightmost curve represents the density of healthy component distribution while leftmost curve represents the density of faulty component distribution. The separation tells the resolving power of modified EM method.

2. Yield: The yield in Monte Carlo simulator can be changed to simulate high and low yield wafers. For low yield wafers, the faulty component distribution will have more tau samples than the healthy component distribution. Figure 3.8 shows the simulated effect of yield on tau distributions. 100 dies are taken as input to simulator with a yield of $40 \%$ and $90 \%$.

3. Reduced number of input states: The rounding of tau sample to 4, 3, 2 and 1 decimal places is done to simulate the effect of reduced number of test input states. If $s$ is the number of input test states per die, the tau calculated for die is inversely proportional to $s(s-1)$. If number of states, $s$ is reduced, the resolution of tau value will decrease to lower number of decimal places which means the data will have more ties. More number of ties mean less resolution for modified EM method to work.

The Monte Carlo simulator synthesizes two random beta distributions to be given as input to modified EM method. However, the production data contains noise such as process variation and tester noise. The tau distribution calculated from wafers in a production dataset will not resemble mixture of two beta distributions. 


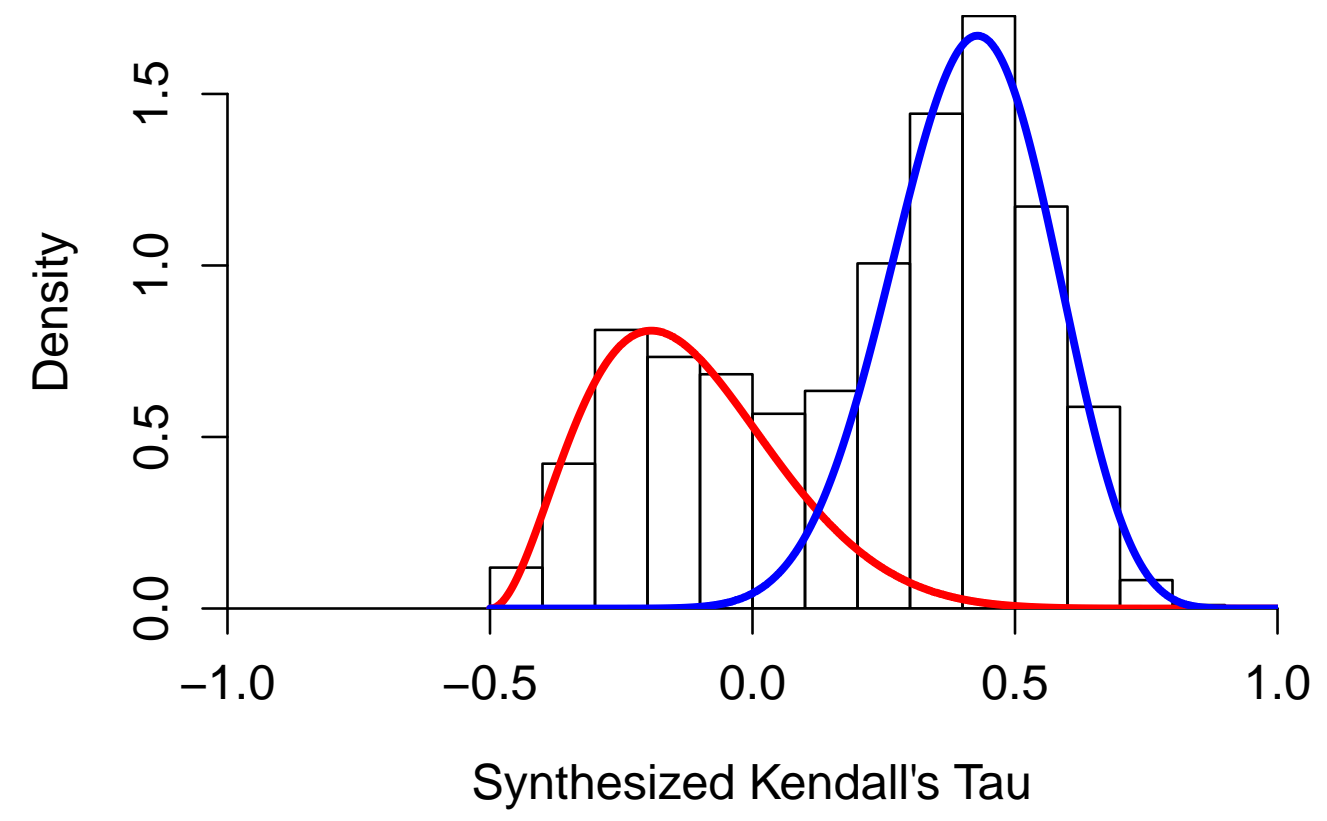

(a) Widely separated healthy and faulty components. Simulated parameters are $\alpha_{H}=14$, $\beta_{H}=8, \alpha_{F}=3, \alpha_{H}=7, p=0.51$.

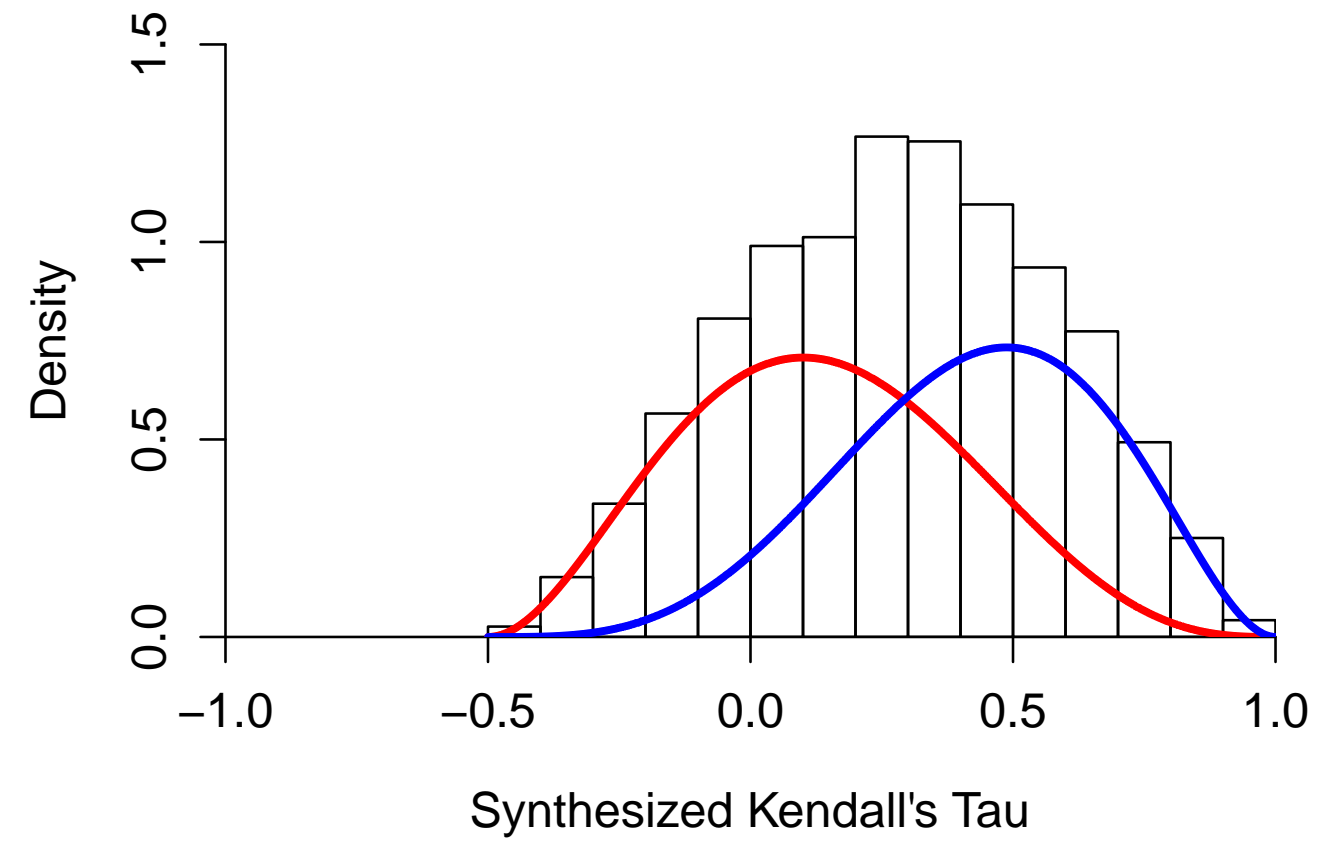

(b) Mixed healthy and faulty components. Simulated parameters are $\alpha_{H}=3, \beta_{H}=4$, $\alpha_{F}=5, \alpha_{H}=3, p=0.51$.

Figure 3.7: Two component mixtures showing examples of varying mixing proportion of healthy and faulty components. 


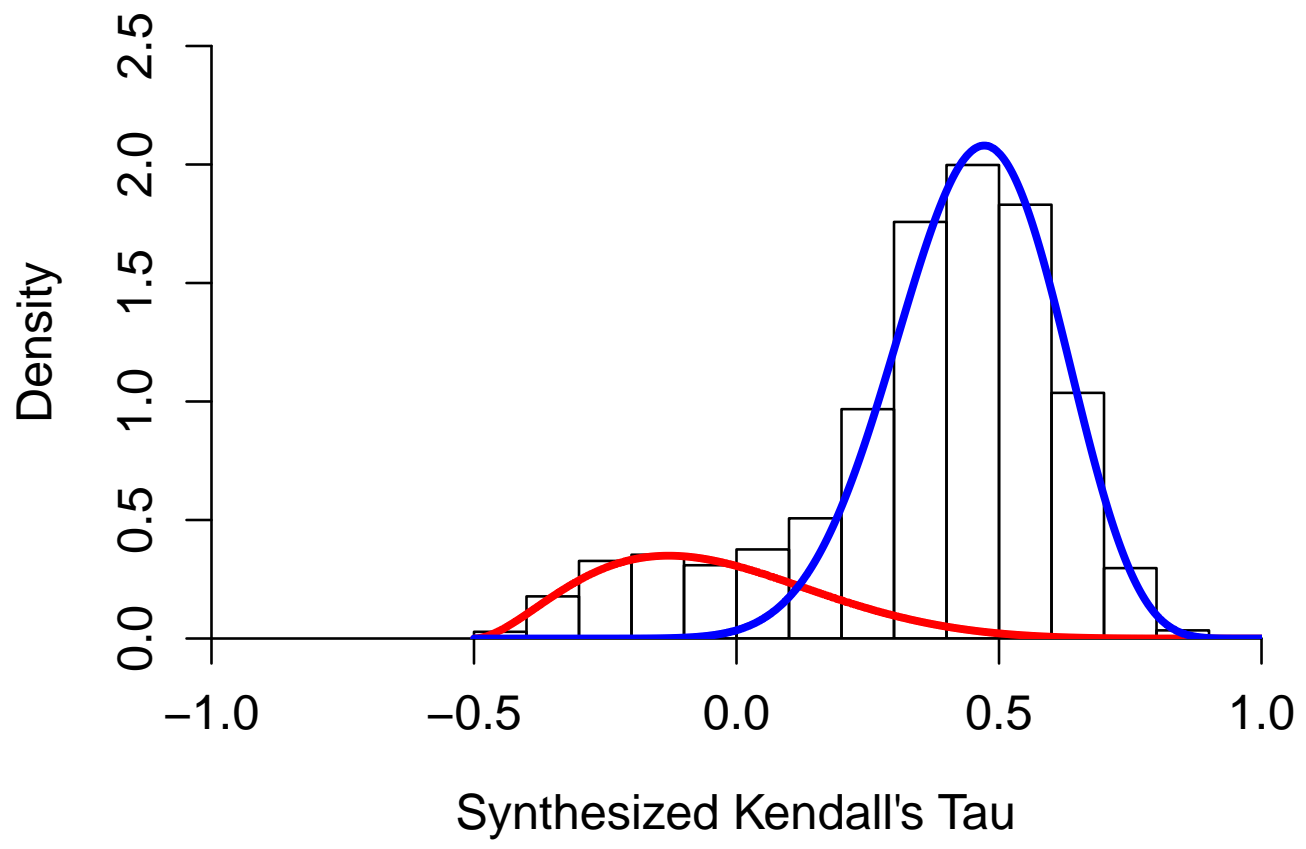

(a) Yield of wafer $=90 \%$. Simulated parameters are $\alpha_{H}=14, \beta_{H}=8, \alpha_{F}=3, \alpha_{H}=7$, $p=0.19$.

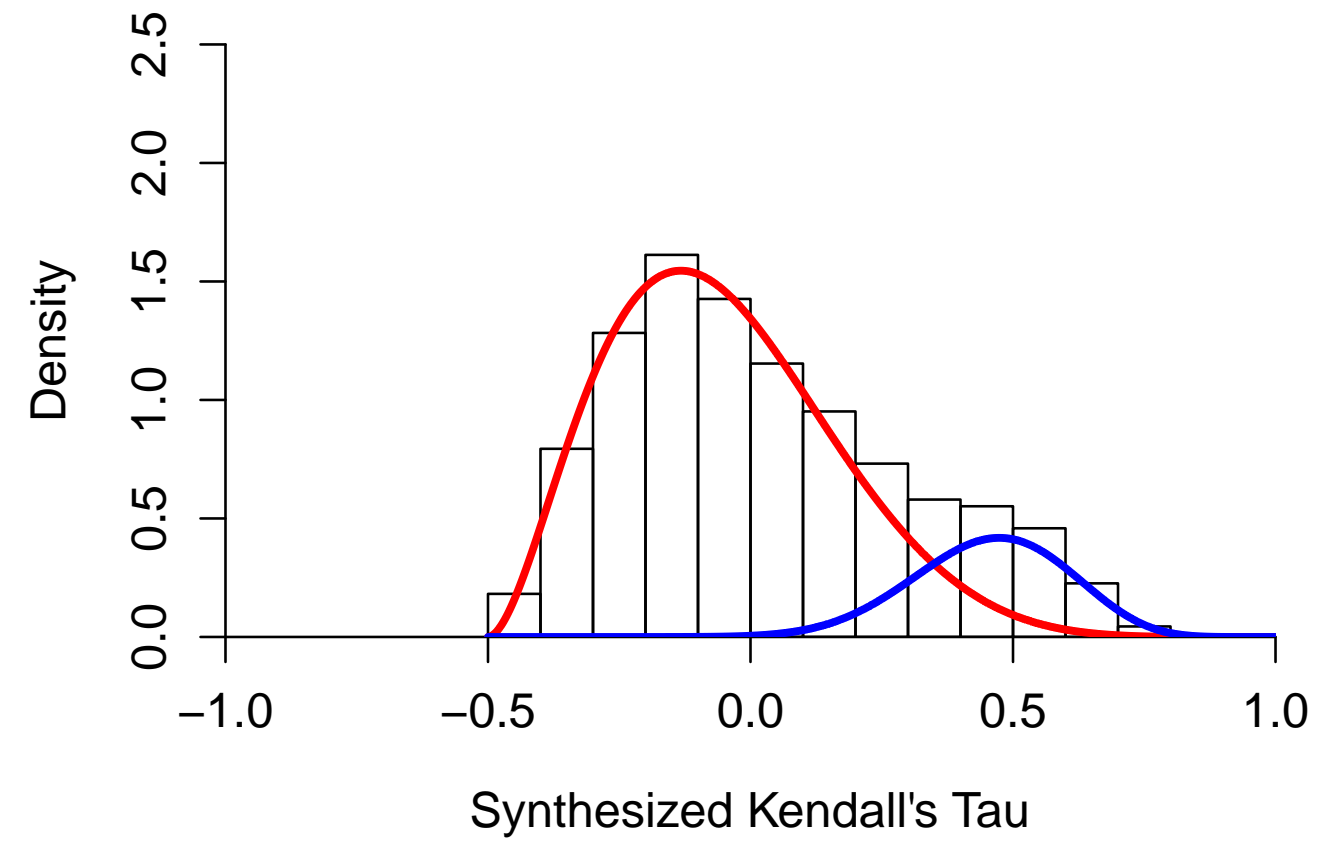

(b) Yield of wafer $=40 \%$. Simulated parameters are $\alpha_{H}=14, \beta_{H}=8, \alpha_{F}=3, \alpha_{H}=7$, $p=0.84$.

Figure 3.8: Beta mixture model showing examples of effect of yield on proportion of healthy and faulty component distributions. 
There is need of a noise model in the Monte Carlo simulator. So, a third beta distribution $\left(\alpha_{C}, \beta_{C}\right)$ is introduced as a noise (contaminant distribution) to both the healthy and faulty components. Figure 3.9 shows the example of a noisy beta distribution (in dotted lines) introduced in the two component beta mixture. The mixture with the noise model is represented as:

$$
\begin{aligned}
f\left(\tau \mid p_{1}, p_{2}, \alpha_{F}, \beta_{F}, \alpha_{H}, \beta_{H}, \alpha_{C}, \beta_{C}\right)= & p_{1} \operatorname{Beta}\left(\alpha_{F}, \beta_{F}\right) \\
& +p_{2} \operatorname{Beta}\left(\alpha_{H}, \beta_{H}\right) \\
& +\left(1-p_{1}-p_{2}\right) \operatorname{Beta}\left(\alpha_{C}, \beta_{C}\right)
\end{aligned}
$$

where $p_{1}$ is the mixing proportion of the faulty component, $p_{2}$ is the mixing proportion of the healthy component and $\alpha_{C}, \beta_{C}$ are the shape parameters of the contaminant distribution. The contamination factor, $\mathrm{C}$ determines the amount of contaminant distribution in the original mixture distribution. For example, if $\mathrm{C}=$ $0.3,30 \%$ of the tau values of healthy component distribution will be replaced by values from contaminant distribution and $30 \%$ of values of faulty component distribution will be replaced by values from contaminant distribution. The contaminant factor $\mathrm{C}$ is a constant value. To introduce more variability in the distribution, $\mathrm{C}$ can be chosen to be a value coming from a normal distribution having a mean $\mathrm{C}$ and some standard deviation. The results of Monte Carlo simulations are described without noise and with noise model in Chapter 4. 


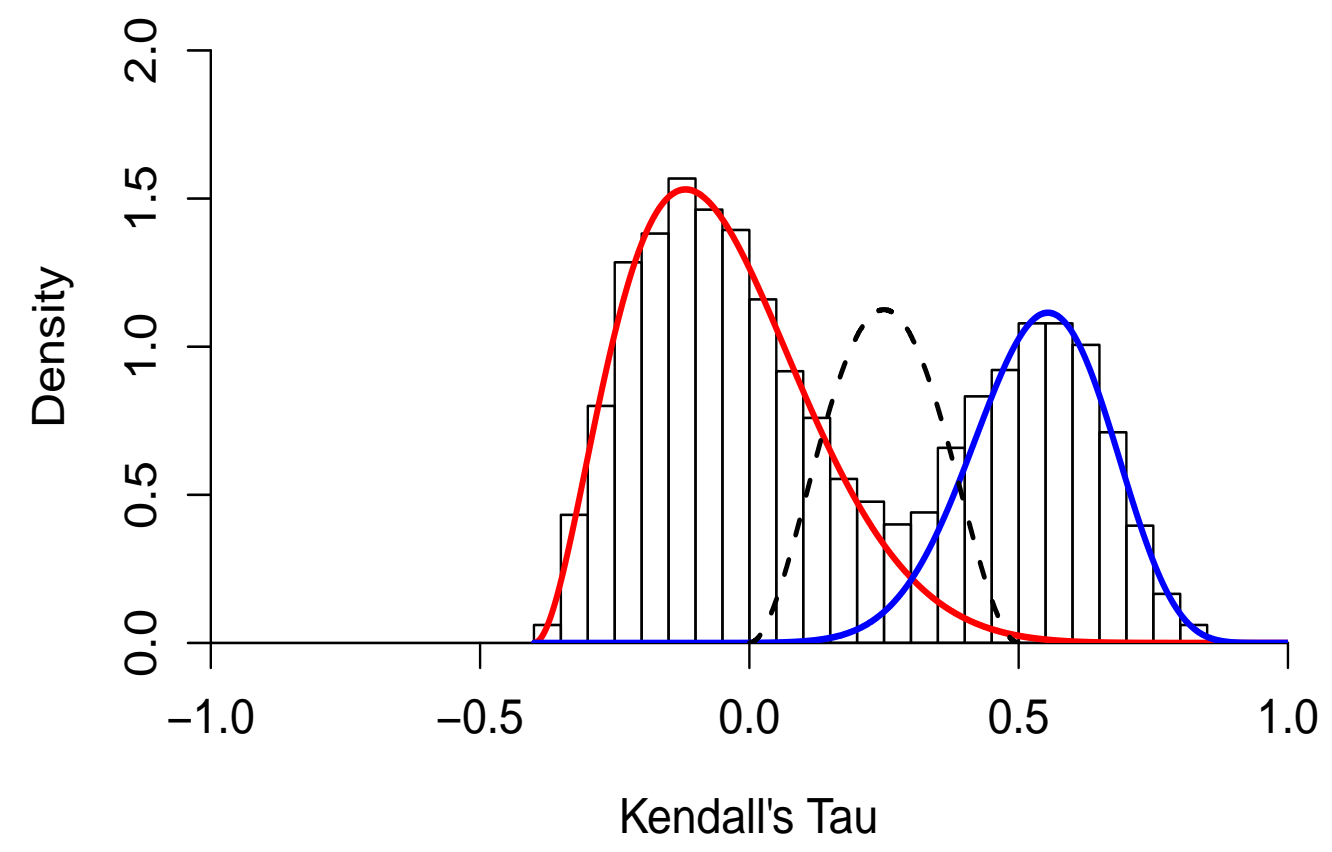

Figure 3.9: An example mixture of healthy and faulty component distributions showing where a contaminant distribution is placed (dotted lines). Simulated parameters are $\alpha_{H}=18, \beta_{H}=9, \alpha_{F}=3, \alpha_{H}=9, p=0.64, \alpha_{C}=3, \beta_{C}=3$. 


\section{Chapter 4}

\section{Results}

\subsection{Monte Carlo Simulation Results}

The Monte Carlo simulation is run on synthesized tau mixtures. The tau mixtures are synthesized using different types of beta distributions including wide, narrow, separated and mixed distributions. The number of dies for synthesized tau mixtures range from 100 to 600 . Synthesized tau mixtures are generated for low yield (20\% yield) wafers to high yield (95\% yield) wafers. By changing all the input parameters, 256 different tau mixtures are synthesized and given to modified EM. The modified EM gives the shape parameters estimate and the squared yield of the mixture. The following subsections describe the overall Monte Carlo results without the noise and with noise model.

\subsubsection{Without Noise}

Figure 4.1 and 4.2 show the correlation between 256 input shape parameters $\alpha$ and $\beta$ and the estimated shape parameters $\alpha$ and $\beta$. For all the different types of input mixtures, correlation between estimated and input parameters is high. The modified EM estimates correctly estimates the input parameters of the underlying beta distributions in the tau mixture distribution.

Figure 4.3 shows the correlation between input yield of the mixture and the estimated yield. The input yield is changed from 20\% to $90 \%$. For high yielding wafers, the method correctly identifies the healthy and faulty dies. As the yield 


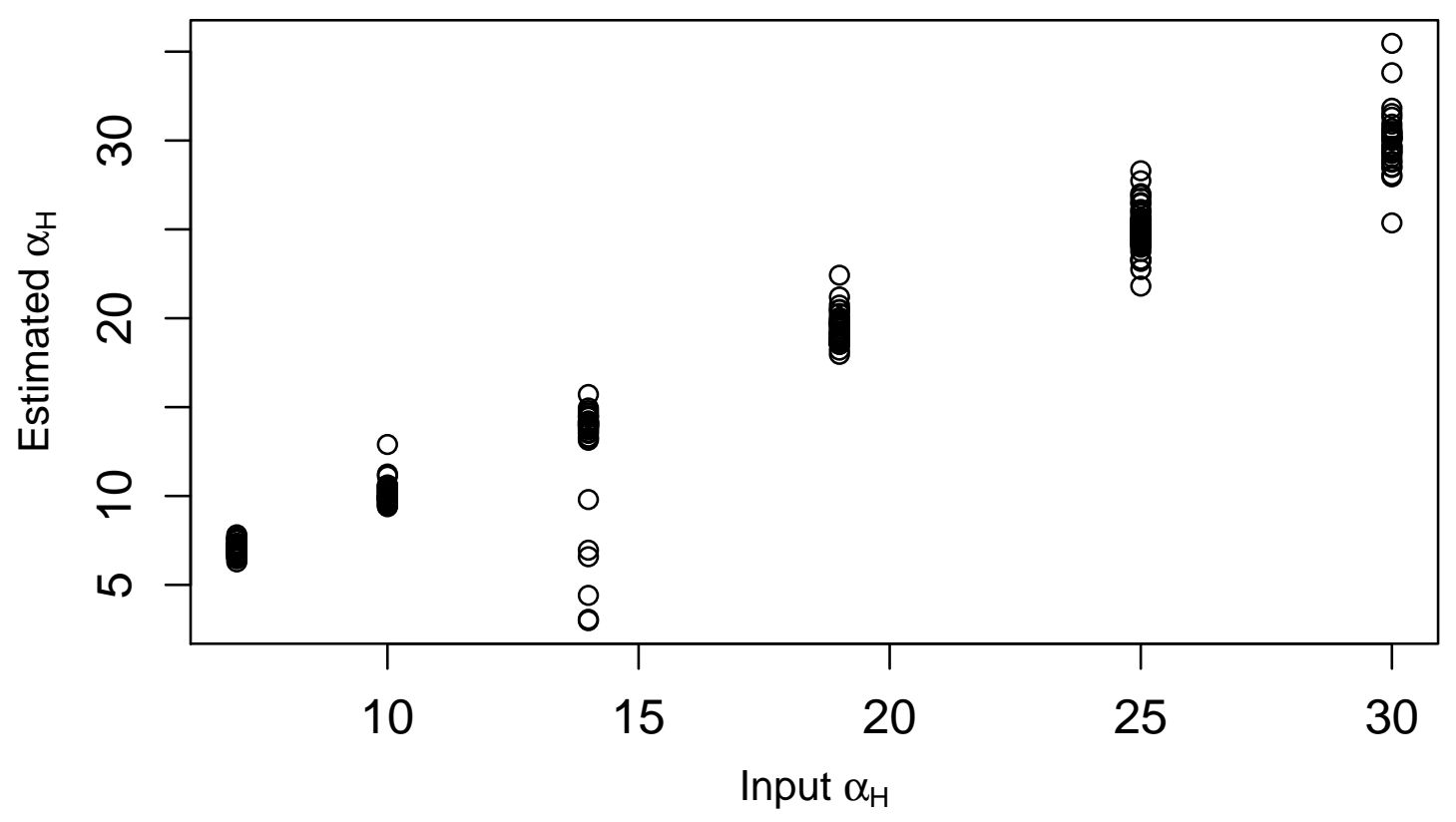

(a) Correlation between input $\alpha_{H}$ and estimated $\alpha_{H}$.

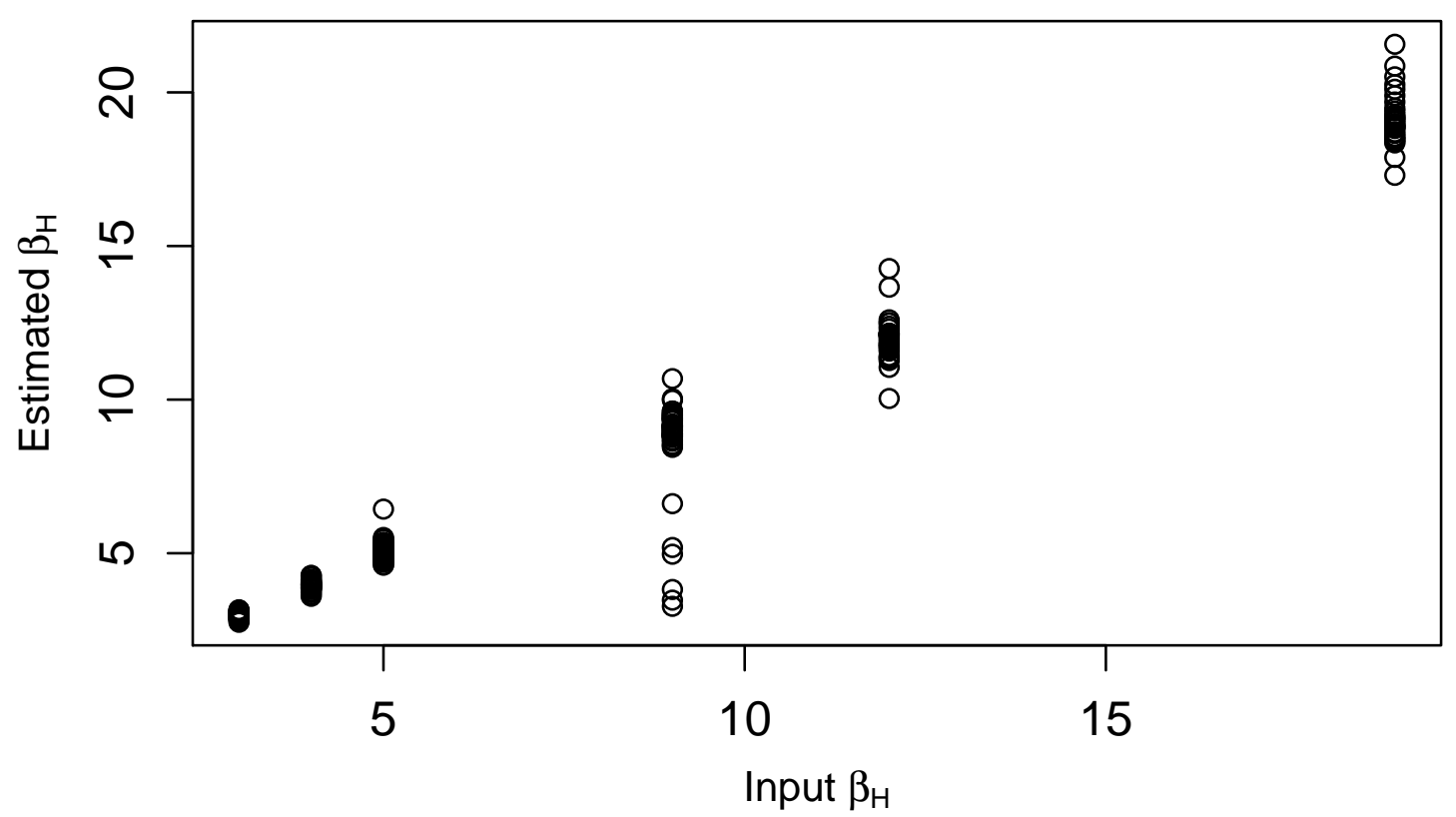

(b) Correlation between input $\beta_{H}$ and estimated $\beta_{H}$.

Figure 4.1: Comparison between input $\alpha$ 's and $\beta$ 's and estimated $\alpha$ 's and $\beta$ 's. 


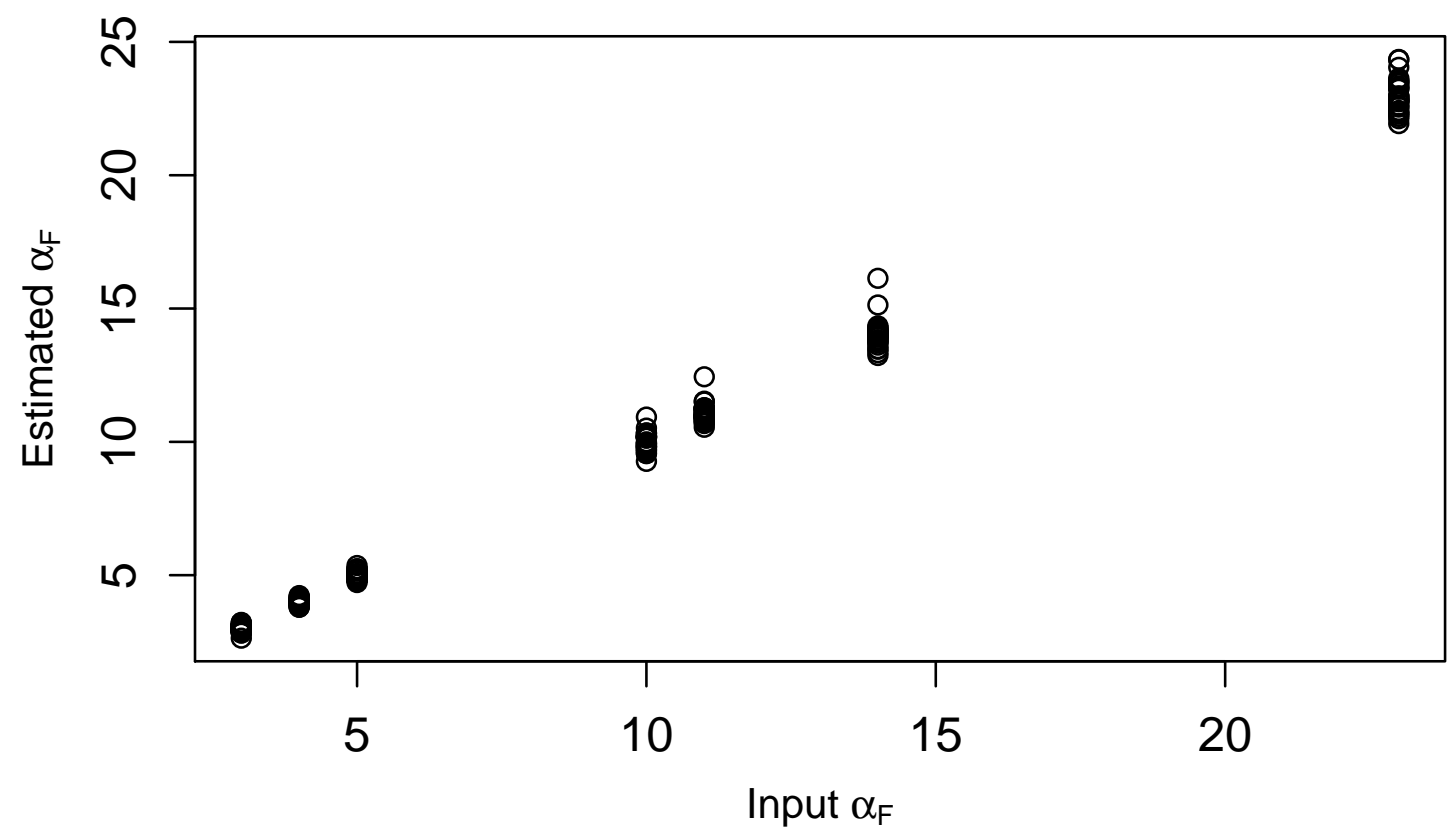

(a) Correlation between input $\alpha_{F}$ and estimated $\alpha_{F}$.

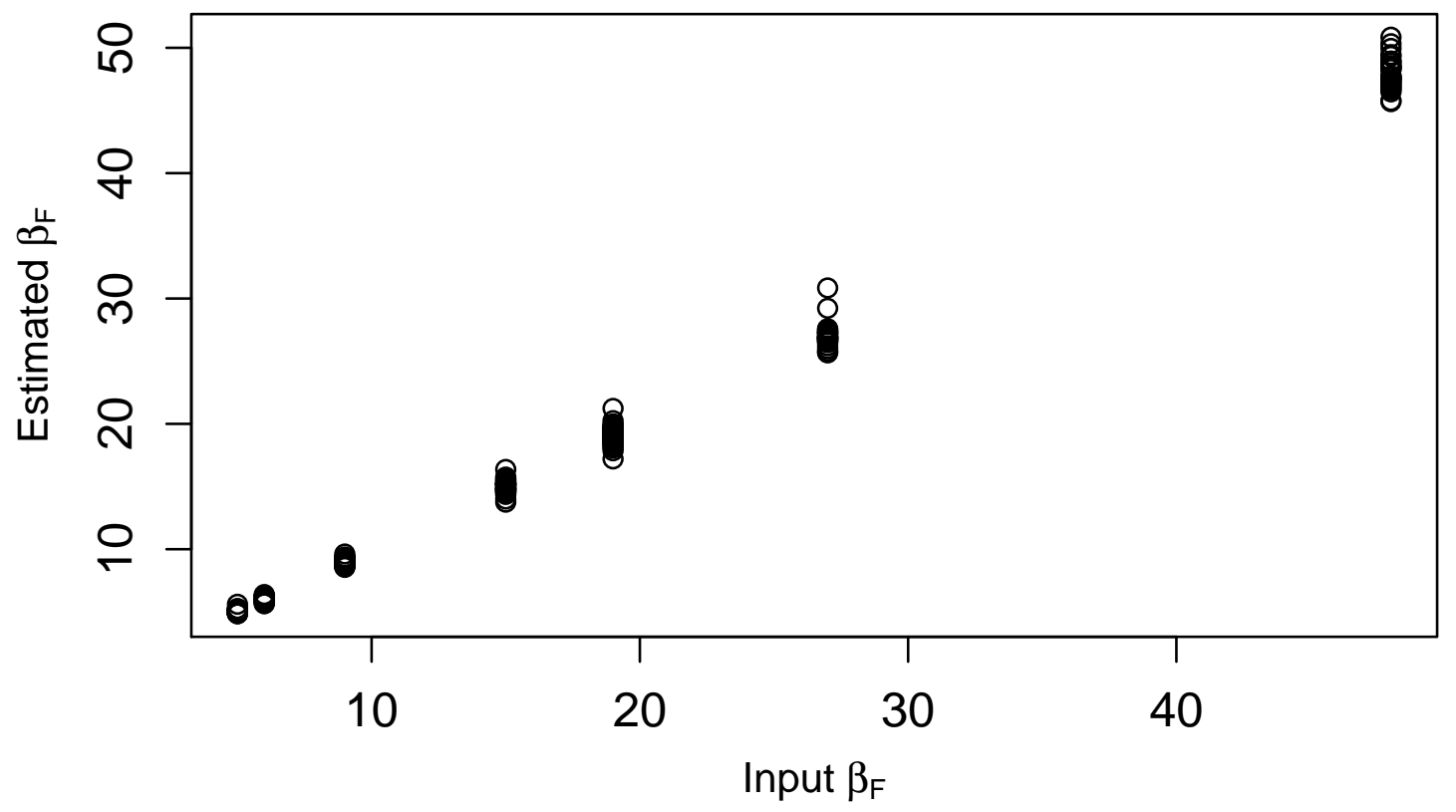

(b) Correlation between input $\beta_{F}$ and estimated $\beta_{F}$.

Figure 4.2: Comparison between input $\alpha$ 's and $\beta$ 's and estimated $\alpha$ 's and $\beta$ 's. 


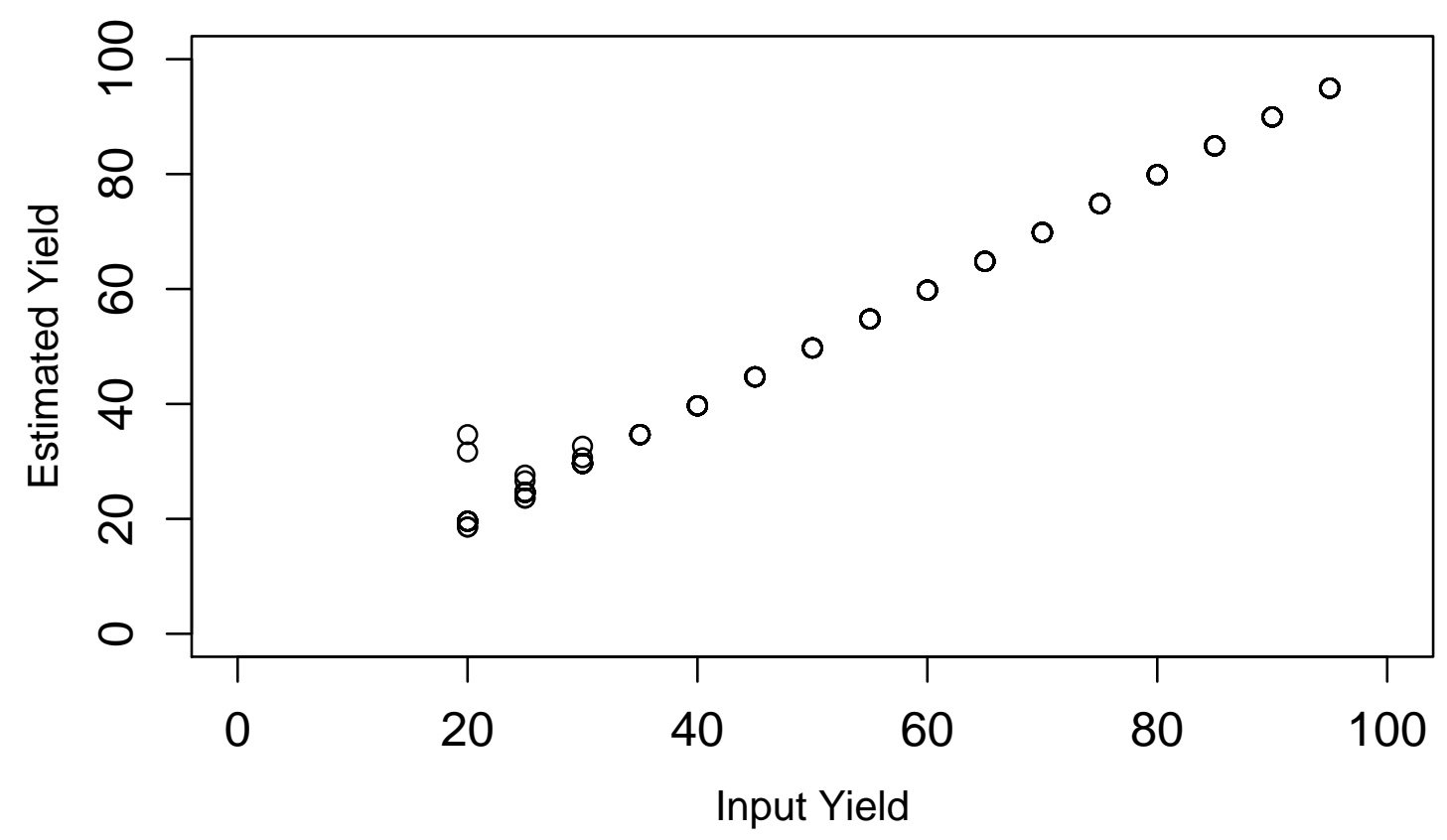

Figure 4.3: Comparison between input yield and estimated yield.

starts decreasing, the faulty distribution has more samples than healthy distribution and the mixture distribution looks like a single healthy distribution. As the yield starts decreasing below 35\%, modified EM starts misclassifying dies as healthy dies.

The modified EM method correctly identifies the faulty dies when the tau are rounded to 4, 3 and 2 places of decimal. However, when the rounding is done to 1 place of decimal, the modified EM method does not work. The modified EM miss-classifies all the dies as healthy due to large number of ties in tau distribution.

\subsubsection{With Noise Model}

Figure $4.4 \mathrm{a}, 4.4 \mathrm{~b}$ and 4.5 show three examples of effects of the contamination factor, $\mathrm{C}$ on the two component tau distribution of Figure 3.9 with $\mathrm{C}=0, \mathrm{C}=0.3$ 
and $\mathrm{C}=0.5$ respectively. With values of $\mathrm{C}$ greater than 0 , the healthy and faulty components start mixing with each other.

Figure 4.6 shows the estimated yield of the 256 input tau mixtures in presence of noisy distributions with $\mathrm{C}=0.31$. Comparing Figure 4.6 with Figure 4.3 , the estimated parameters are not degraded with respect to input parameters. From the value of $\mathrm{C}=0$ to $\mathrm{C}=0.31$, the estimated parameters are almost equal to input parameters except for lower yields. After $\mathrm{C}$ is increased from $\mathrm{C}=0.31$, the estimated parameters gradually degrade with respect to input parameters.

Figure 4.7 shows the correlation between input yield of the mixture and the estimated yield when noise is maximum, $\mathrm{C}=0.5$. The 256 tau mixtures are given as input to the modified EM. The correlation between input and estimated yields is degraded due to variation in estimated yields as compared to input yields. For any given yield from $20 \%$ to $90 \%$, the input and estimated yields are degraded. This degradation is gradual from examples shown in Figure 4.3 and Figure 4.6. When estimated yields are degraded, the estimated shape parameters are also degraded with respect to the input shape parameters.

Figures 4.8 and 4.9 show the degraded correlation between 256 input shape parameters $\alpha$ and $\beta$ and estimated shape parameters $\alpha$ and $\beta$ in presence of noise with $\mathrm{C}$ $=0.5$. Figures 4.8 and 4.9 show the case of maximum contamination factor where $\mathrm{C}=0.5$. Above $\mathrm{C}=0.5$, the contaminant distribution becomes the originating distribution.

As the noise level is increased, the healthy and faulty distribution start mixing with each other and modified EM starts misclassifying healthy dies as faulty and faulty 


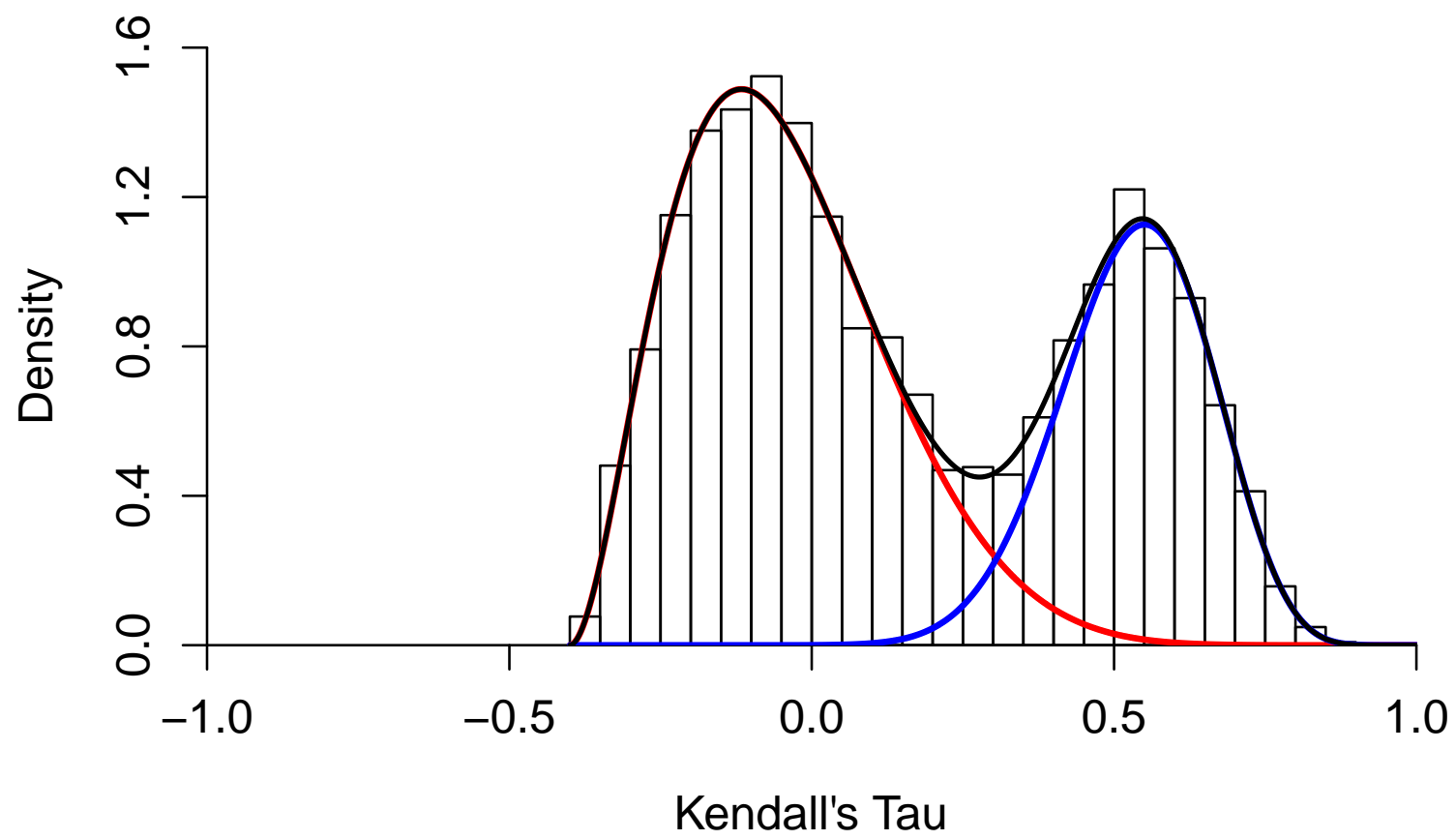

(a) Tau Distribution with $\mathrm{C}=0$.

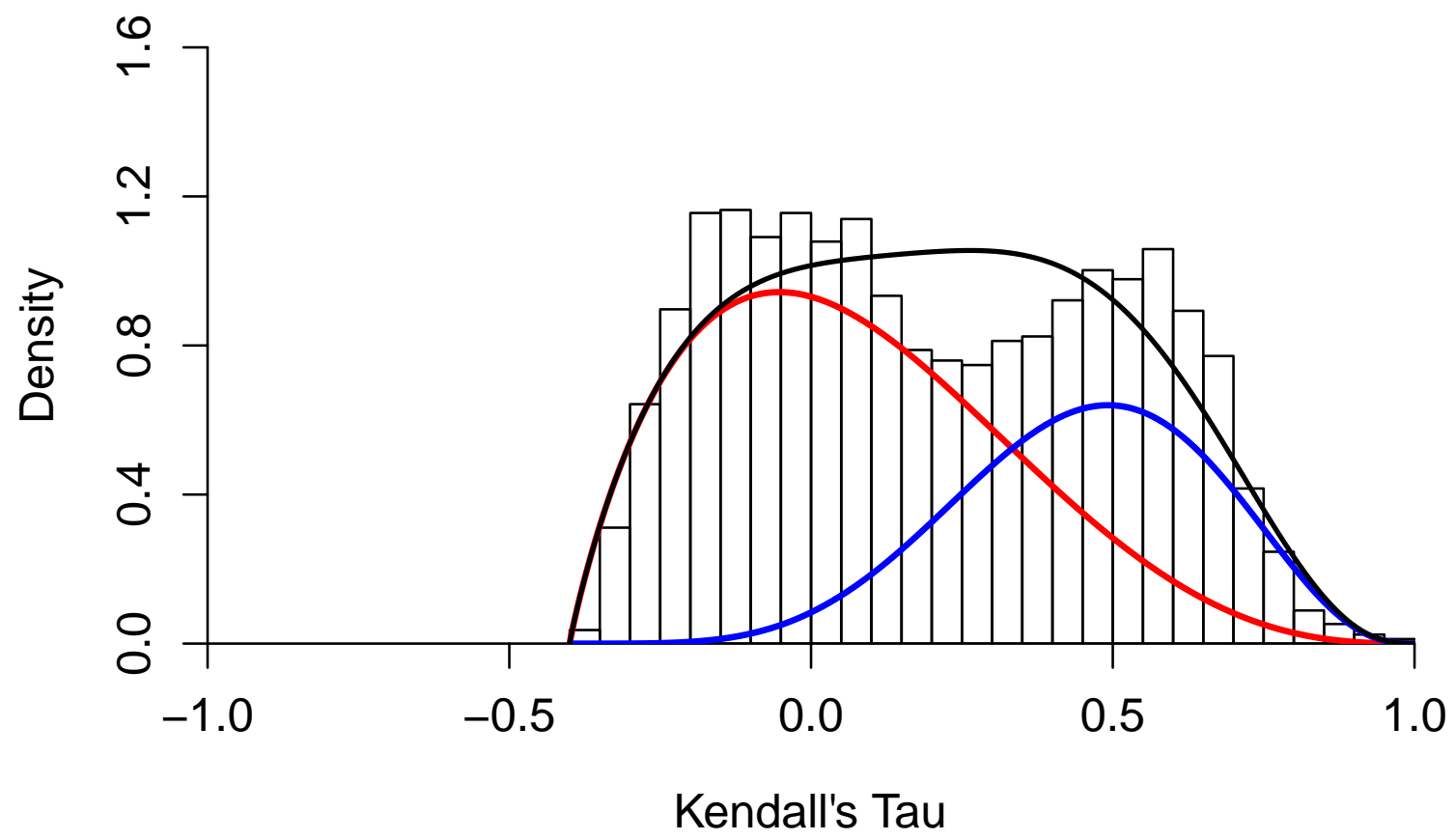

(b) Tau Distribution with $\mathrm{C}=0.31$.

Figure 4.4: Tau histograms with and without noise. Simulated parameters are $\alpha_{H}=18, \beta_{H}=9, \alpha_{F}=3, \alpha_{H}=9, p=0.64, \alpha_{C}=3, \beta_{C}=3$. 


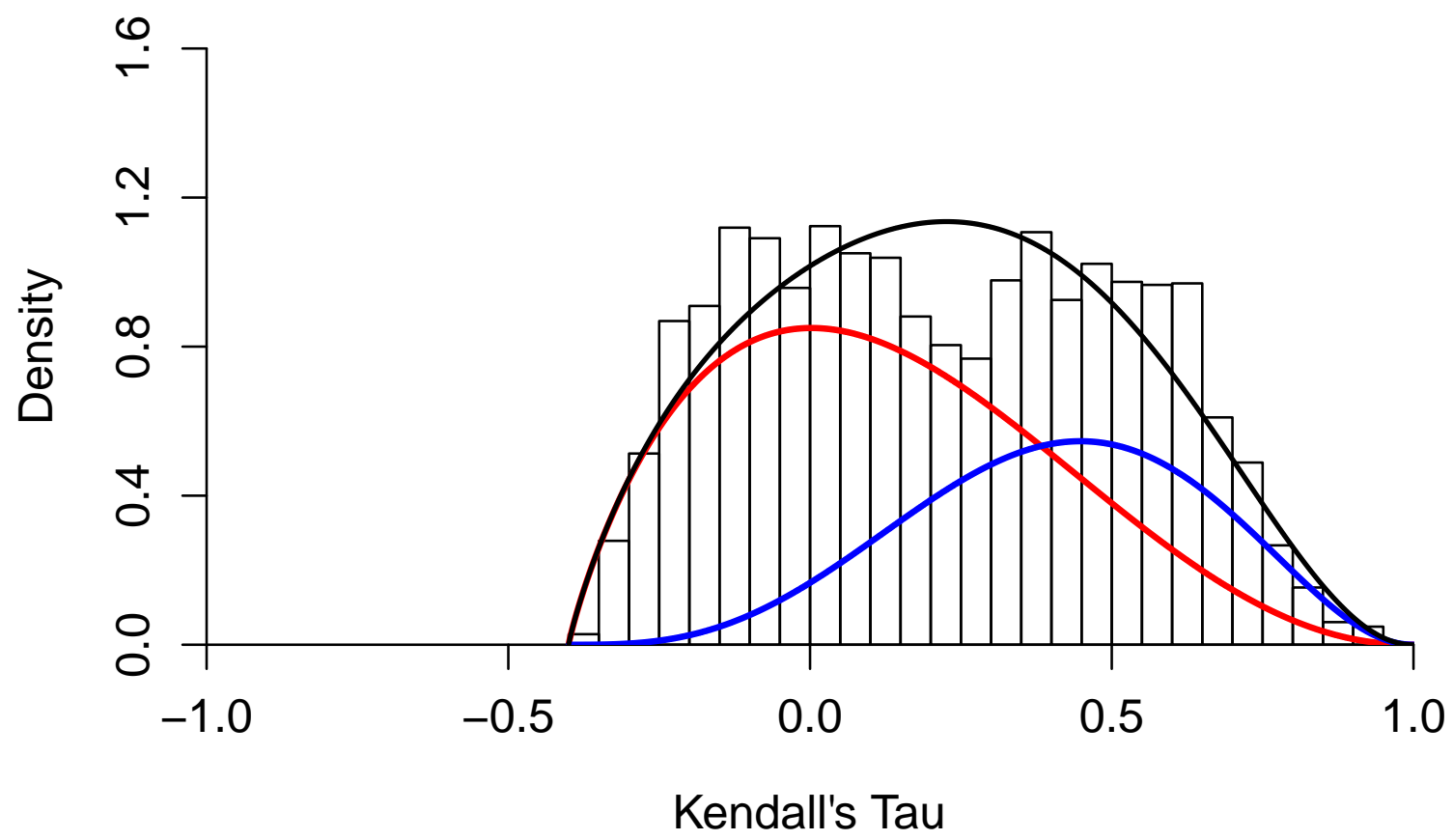

Figure 4.5: Tau histogram with maximum noise factor, $\mathrm{C}=0.5$. Simulated parameters are $\alpha_{H}=18, \beta_{H}=9, \alpha_{F}=3, \alpha_{H}=9, p=0.64, \alpha_{C}=3, \beta_{C}=3$. 


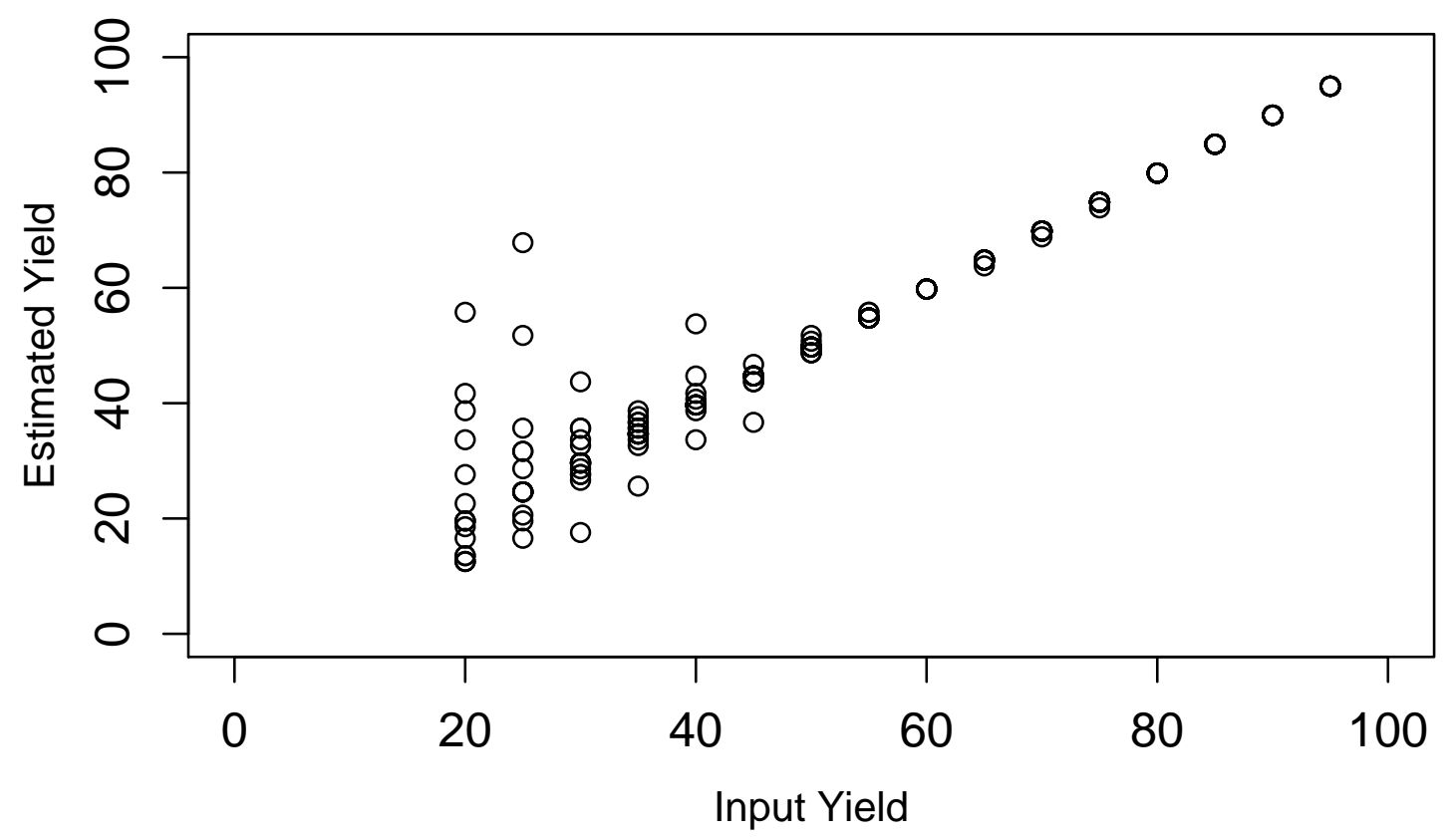

Figure 4.6: Comparison between input yield and estimated yield in presence of noise with $\mathrm{C}=0.31$. The Figure 4.6 can be compared to Figure 4.3. 


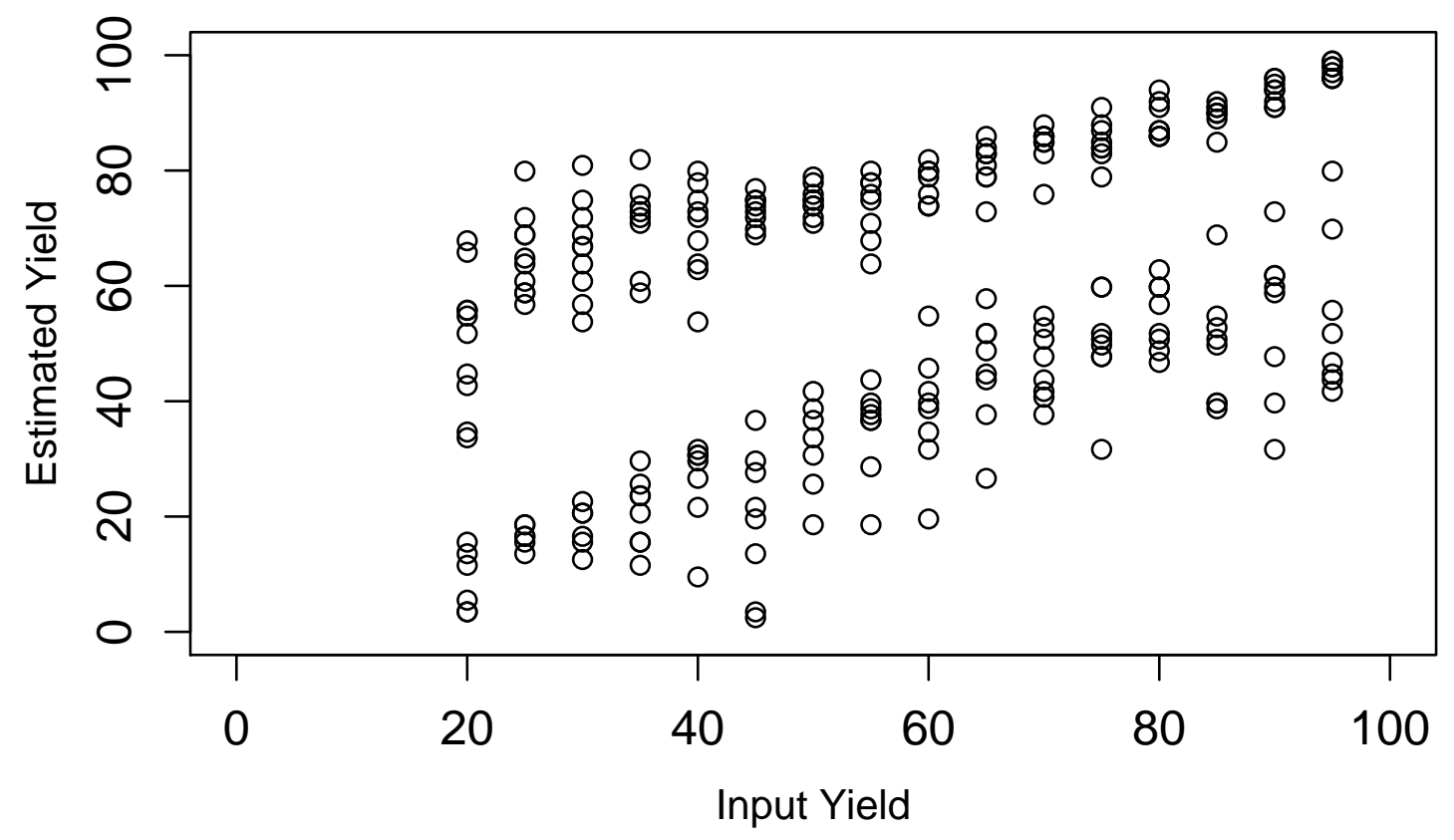

Figure 4.7: Comparison between input yield and estimated yield in presence of noise with $\mathrm{C}=0.5$. 


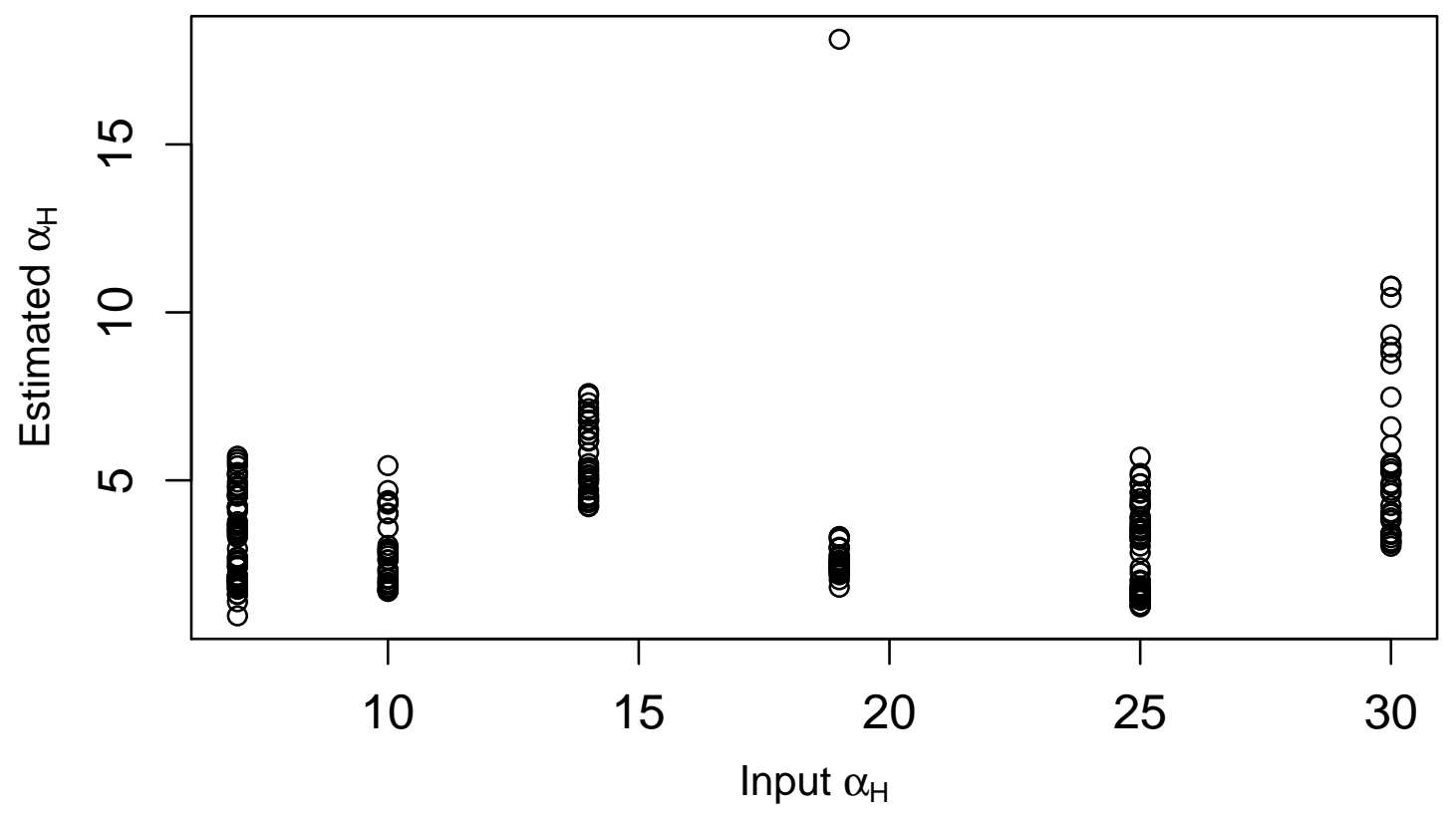

(a) Correlation between input $\alpha_{H}$ and estimated $\alpha_{H}$.

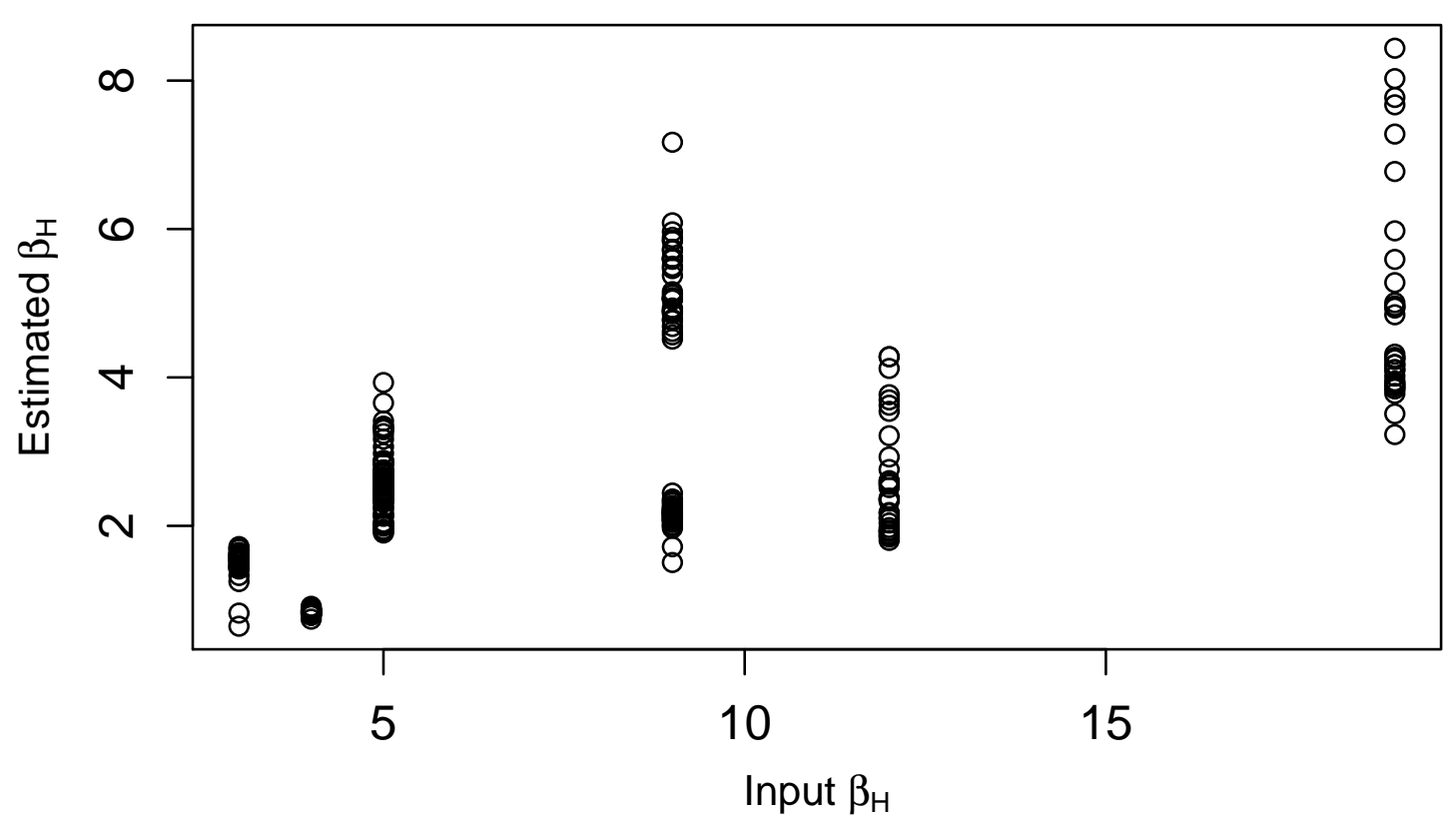

(b) Correlation between input $\beta_{H}$ and estimated $\beta_{H}$.

Figure 4.8: Comparison between input $\alpha$ 's and $\beta$ 's and estimated $\alpha$ 's and $\beta$ 's with $\mathrm{C}=0.5$. 


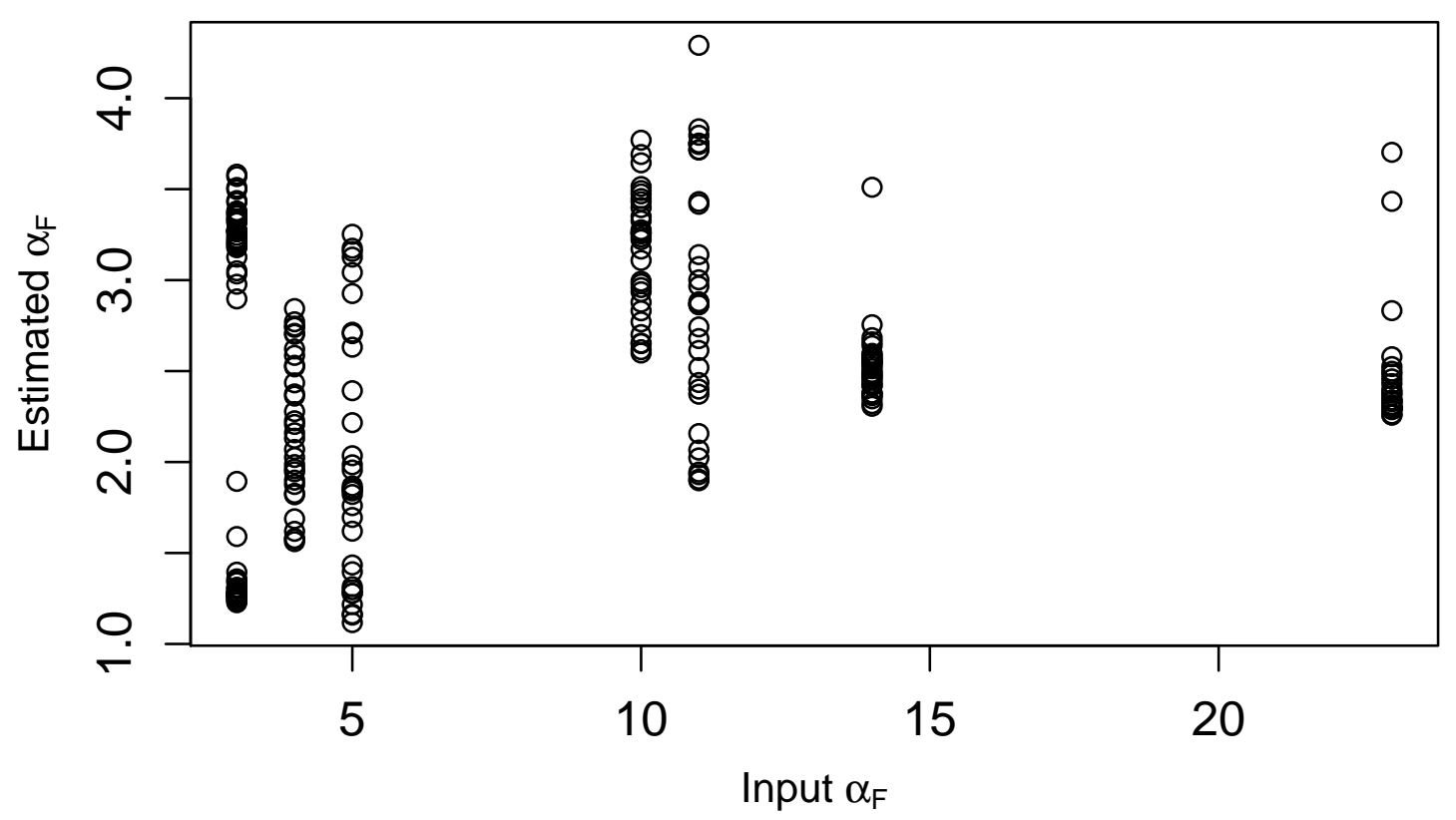

(a) Correlation between input $\alpha_{F}$ and estimated $\alpha_{F}$.

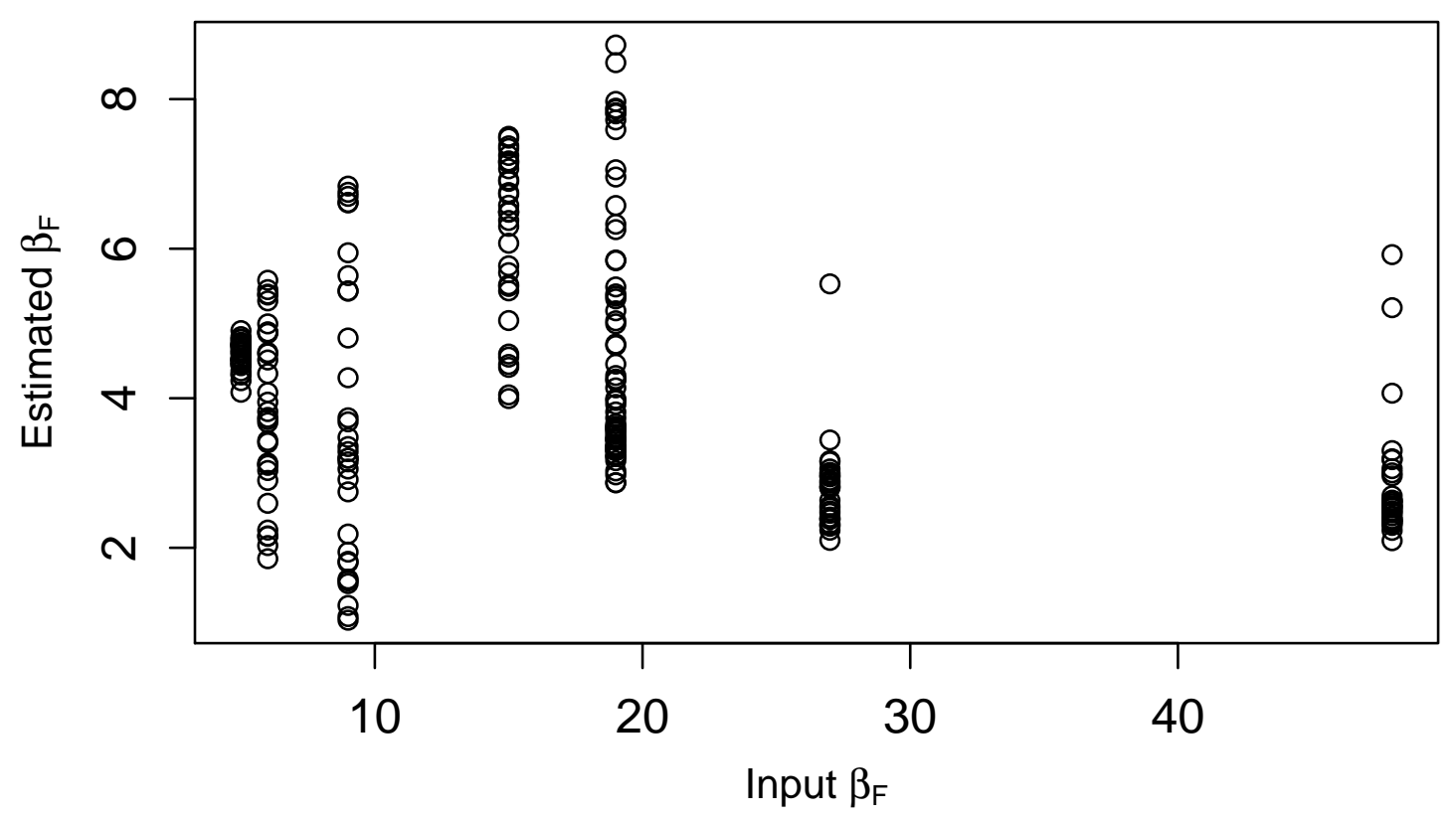

(b) Correlation between input $\beta_{F}$ and estimated $\beta_{F}$.

Figure 4.9: Comparison between input $\alpha$ 's and $\beta$ 's and estimated $\alpha$ 's and $\beta$ 's with $\mathrm{C}=0.5$. 
dies as healthy. The correlation between the input and estimated shape parameters is low for both healthy and faulty distributions. The reason is mixture distribution is a mixture of three distributions instead of two beta distributions. The Monte Carlo simulation examples show that the modified EM method estimates correct input shape and yield parameters. However, increasing noise results in combining of healthy and faulty distributions which mean misclassifying of healthy and faulty dies. Monte Carlo simulations also show low correlation for yields lower than $40 \%$ between estimated and input parameters. Yields lower than $40 \%$ are usually rare in production wafers.

\subsection{Application on Production Data}

\subsubsection{Data Description}

Product $\mathrm{A}$ is a $90 \mathrm{~nm}$ memory ASIC with 4 lots and total number of 4546 dies. Lot 1-4 has 25, 17, 24 and 25 wafers respectively. Each wafer has average number of 60 dies. The dies are tested at two temperatures - (high temperature) $85^{\circ} \mathrm{C}$ and (low temperature) $10^{\circ} \mathrm{C}$. Dies are first tested at $85^{\circ} \mathrm{C}$ and then the dies passing wafer-sort at $85^{\circ} \mathrm{C}$ are passed to $10^{\circ} \mathrm{C}$. Failing dies at $85^{\circ} \mathrm{C}$ are rejected and are not tested further downstream. Each die has pre-stress 64 IDDQ measurements and 64 post-stress IDDQ measurements for both temperatures. Each die consists of 4.2M transistors and has 460 signal IOs, $58 \mathrm{~K}$ bits of register arrays, 29774 LSSD

latches and 25 scan chains. Each die has an embedded DRAM with capacity of 344MBits. The low Vt transistors are $4 \%$ of total number of transistors.

Product B is a 130nm ASIC with 2 wafers having a total of 1068 dies. Each die has 256 IDDQ measurements. The Product B is tested only at a single temperature. 
The IDDQ test is used as the state dependent parametric test for calculation of tau for each wafer of Product $\mathrm{A}$ and $\mathrm{B}$. The wafer-sort test data at $85^{\circ} \mathrm{C}$ (high temperature) of Product $\mathrm{A}$ is taken for calculations. For each wafer of Product A, tau is calculated using the 64 IDDQ pre stress measurements of die pairs. For Product B, 256 measurements of IDDQ are used to calculate tau between die pairs. Figure 4.10 shows the tau histograms for two example wafers from Product A. Figure $4.10 \mathrm{a}$ and $4.10 \mathrm{~b}$ show the tau histogram calculated from two example wafers of Lot 1 from Product A. Figure 4.11 shows the tau histogram calculated from a wafer of Product B.

The tau distributions calculated for each wafer of Product A and B are given input to modified EM method. The modified EM gives the probability of each tau sample belonging to healthy or faulty distribution. Using the tau probability, defective probability of a die is calculated.

\subsubsection{Defective Probability Plots}

Figure 4.12 shows the defective probability plots of two example wafers from Product $\mathrm{A}$. The $\mathrm{x}$-axis represents the die index of a wafer. The $\mathrm{y}$-axis represents the defective probability of each die. The triangles in the probability plot represent dies which are $85^{\circ} \mathrm{C}$ wafer-sort passing dies. The squares represent the $85^{\circ} \mathrm{C}$ wafer-sort failing dies. Dies will be tagged as modified EM fails above a probability threshold of 0.8 represented by a dotted blue line.

In Figure 4.12a, the white space between low probability and high probability dies is quite evident. Most of the $85^{\circ} \mathrm{C}$ wafer-sort passing dies (triangles) lie in the low probability region with probability ranging from 0.2 to 0.5 . In the probability 


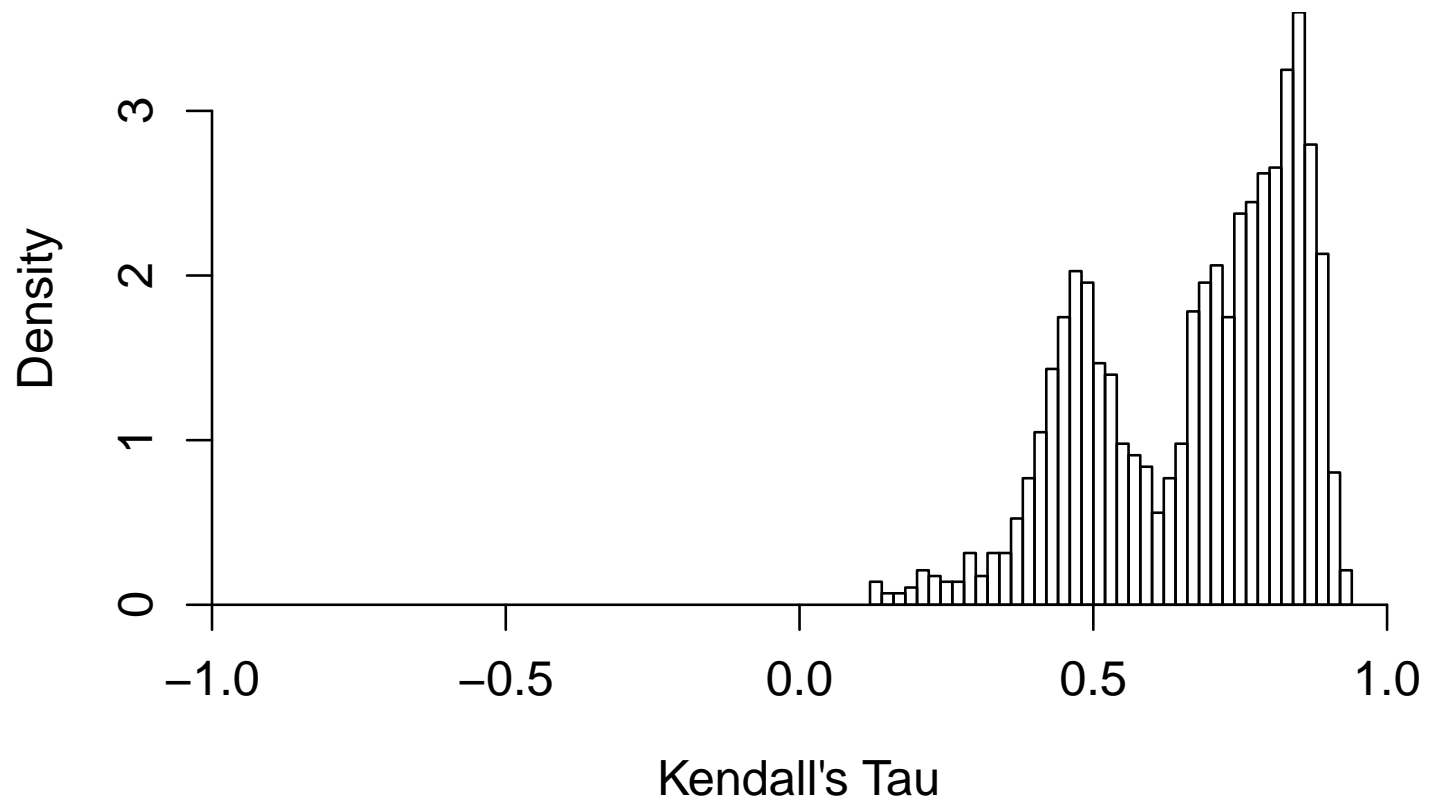

(a) Product A - example wafer 1.

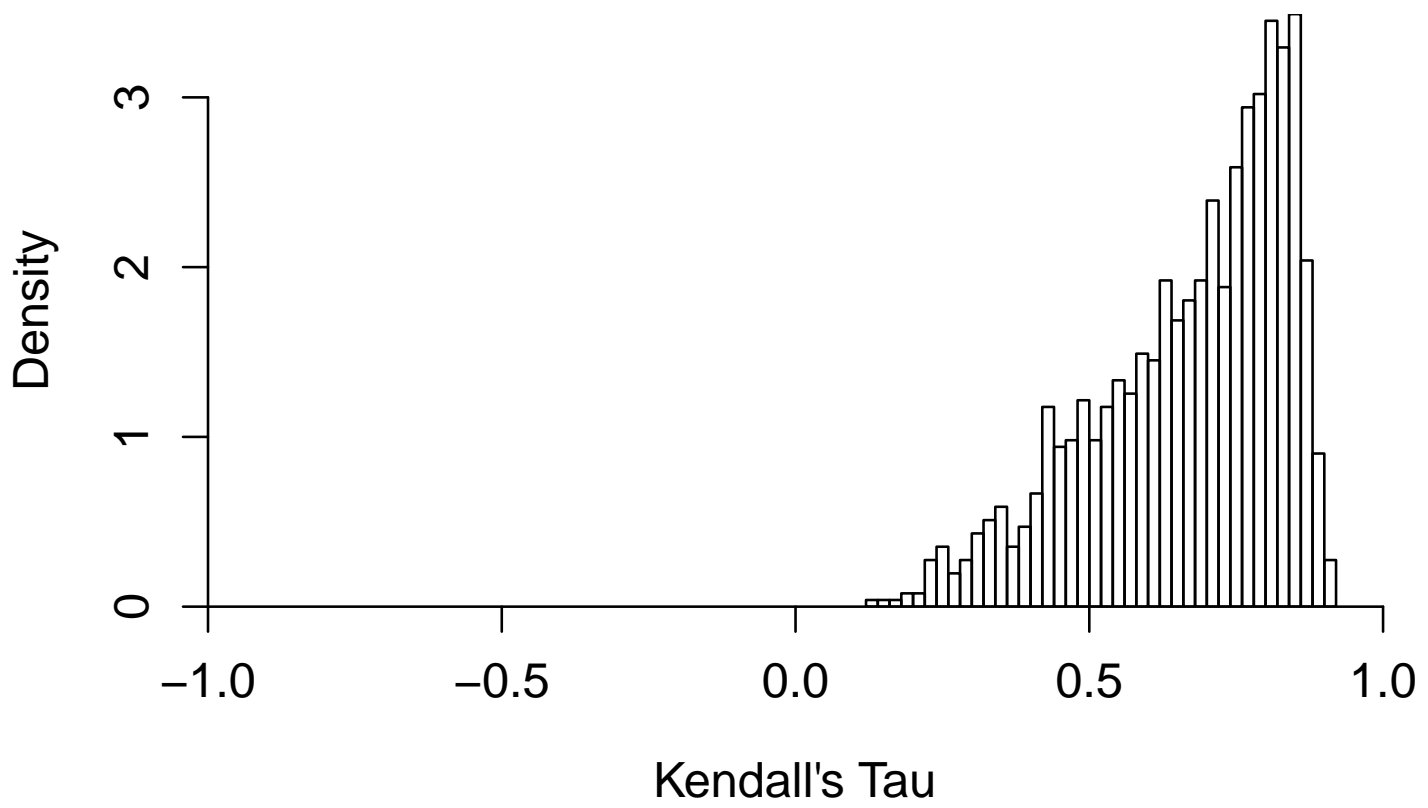

(b) Product A - example wafer 2.

Figure 4.10: Tau histograms of two example wafers from Product A. 


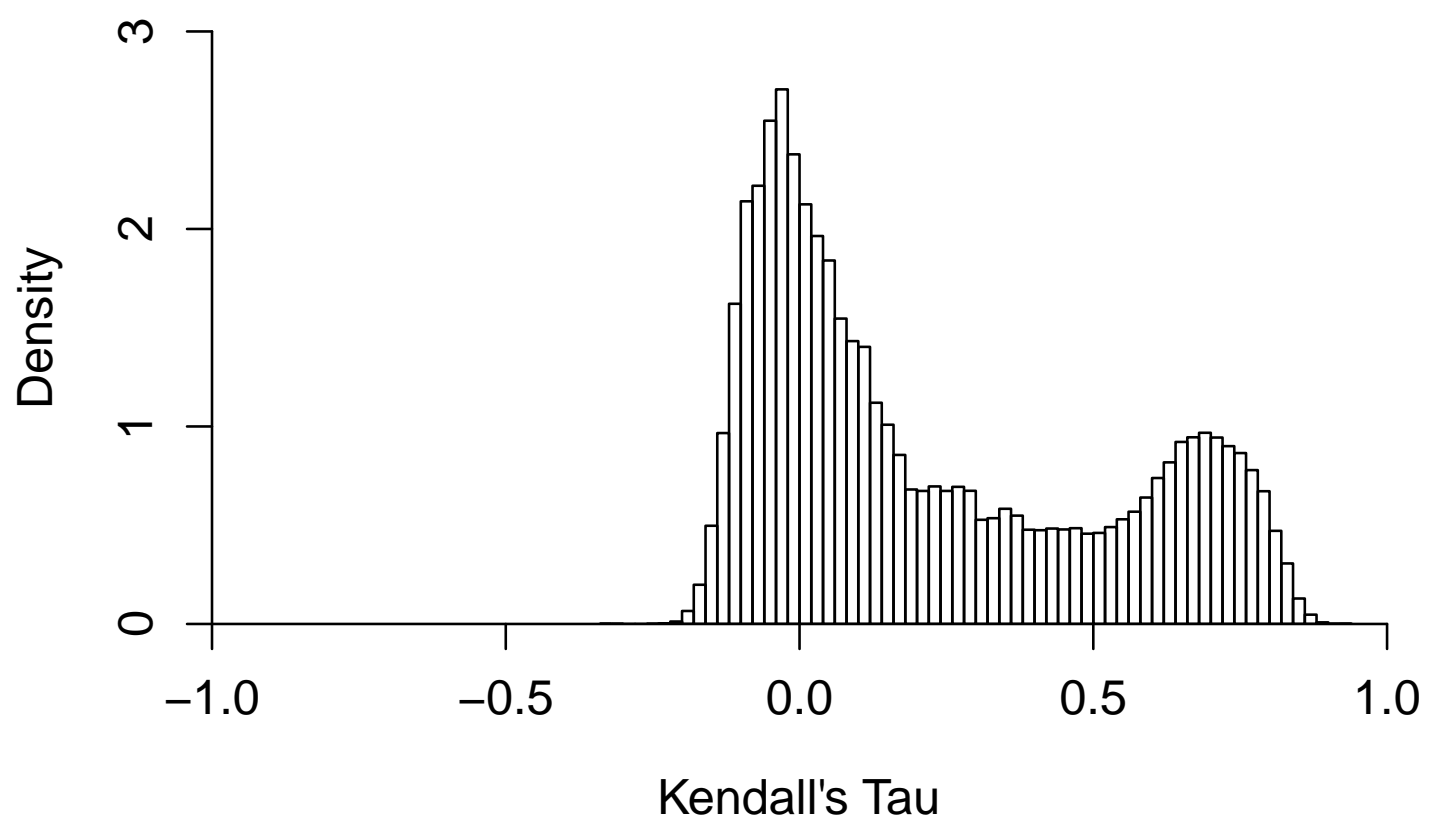

Figure 4.11: Tau histogram of an example wafer from Product B.

range from 0.5 to 0.8 , both $85^{\circ} \mathrm{C}$ wafer-sort passing and failing dies are scattered. Above probability $=0.8$, there is a group of wafer-sort failing dies and four wafersort failing dies. The four dies are tagged as fails by modified EM method. Out of four dies tagged as fails, one die failed in downstream pattern test at $10^{\circ} \mathrm{C}$.

The pass/fail probability threshold for a die is difficult to decide as a constant for all wafers. The defective probability of each die depends upon the tau sample probability of belonging to healthy/faulty distribution. A healthy/faulty distribution can vary for different wafers depending upon the correlation between test values of die pairs. Four probability thresholds of $0.95,0.90,0.85$ and 0.80 will be used in this thesis for tagging a die being modified EM faulty. 


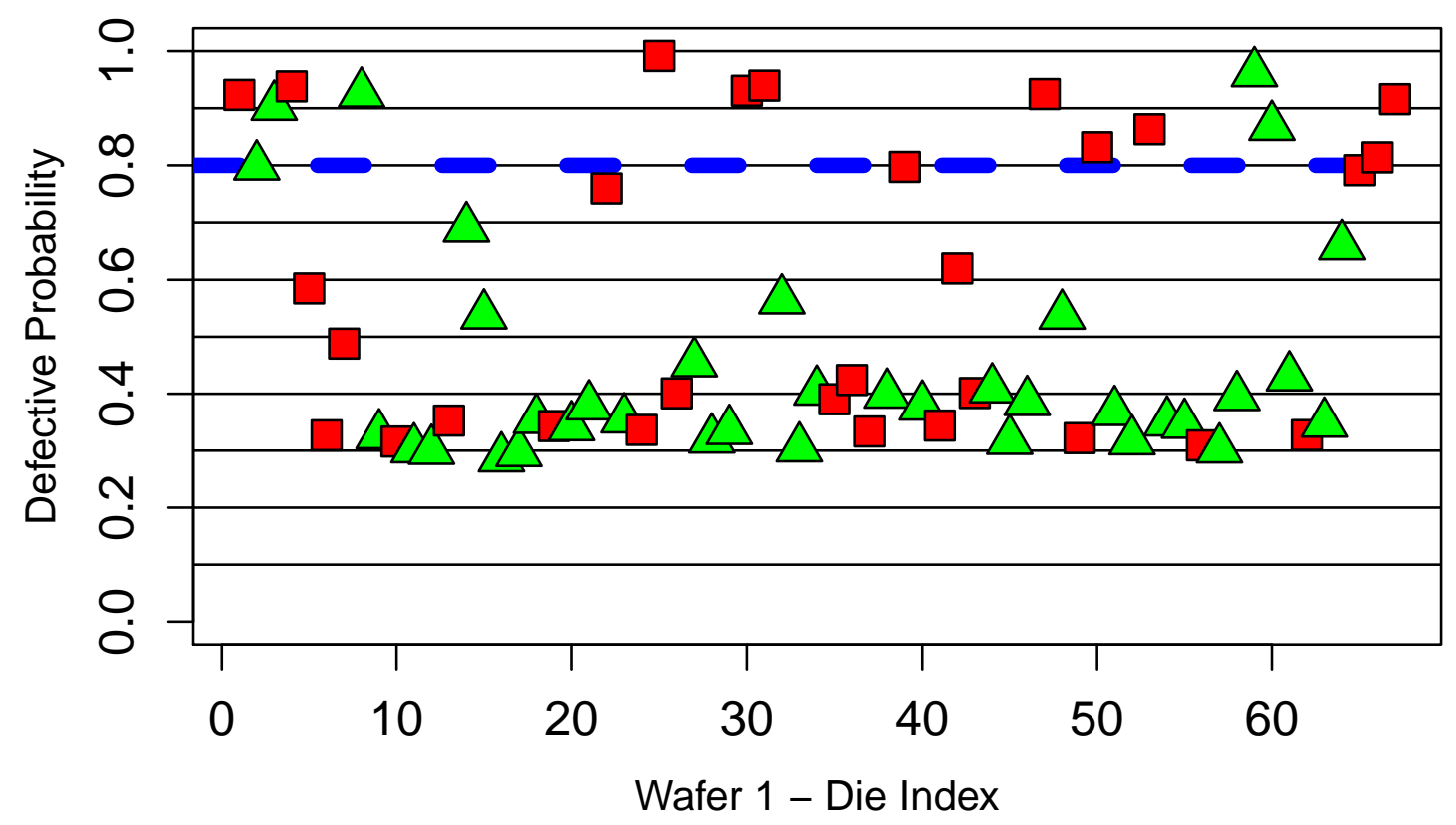

(a) Product A - example wafer 1.

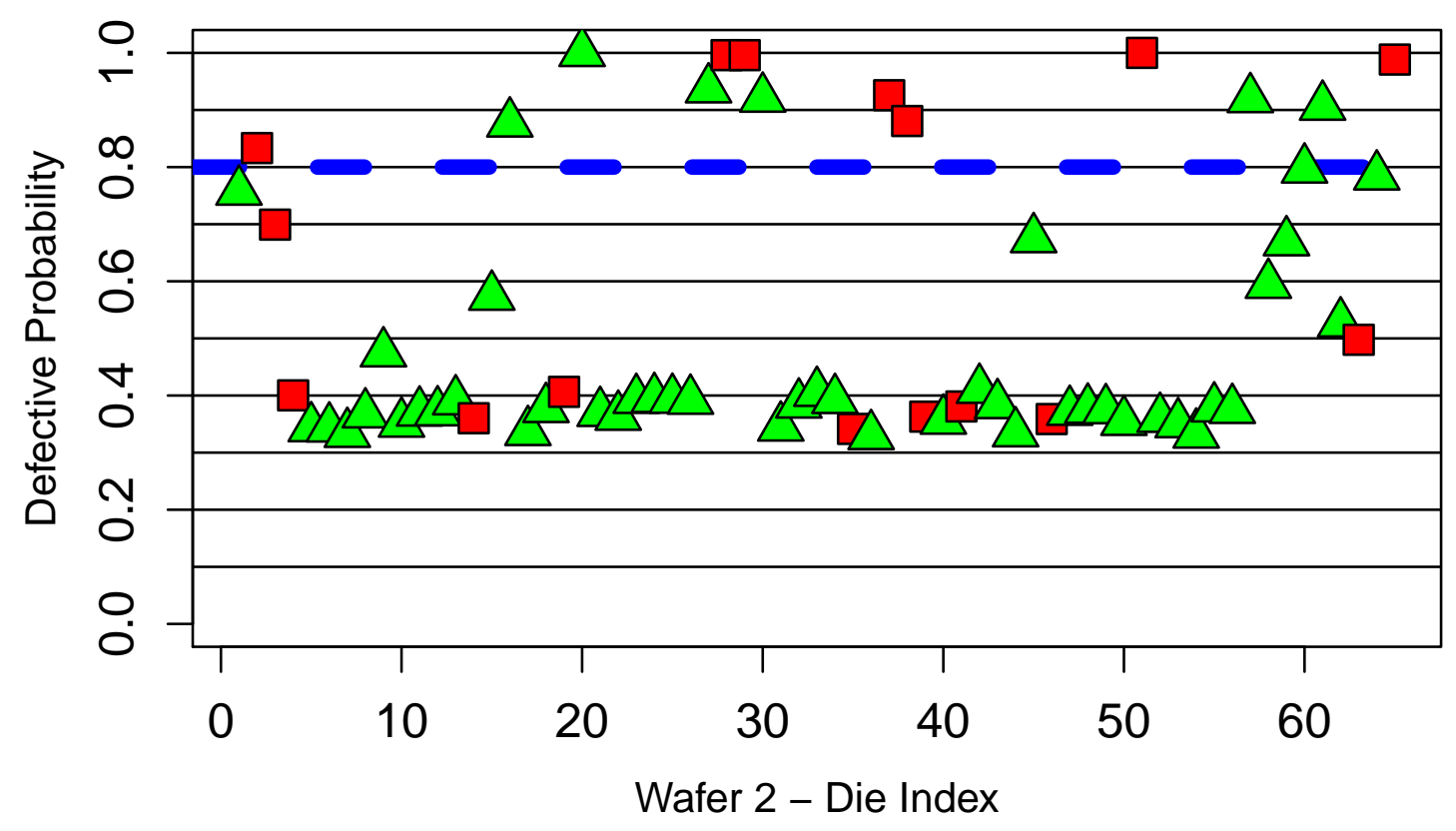

(b) Product A - example wafer 2.

Figure 4.12: Defective probability plots of two example wafers of Product A. Triangles are $85^{\circ} \mathrm{C}$ wafer-sort passes while squares are $85^{\circ} \mathrm{C}$ wafer-sort fails. 
In Figure $4.12 \mathrm{~b}$, most of the $85^{\circ} \mathrm{C}$ wafer-sort passing dies (triangles) lie in the low probability region from 0.3 to 0.5 . There are ten dies scattered in the region between 0.5 and 0.8 . There are six $85^{\circ} \mathrm{C}$ wafer-sort passing dies above 0.8 which will be tagged as fails by modified EM method. Out of six dies tagged as fails, one failed downstream $10^{\circ} \mathrm{C}$ delay test and one failed downstream $10^{\circ} \mathrm{C}$ IDDQ test.

Dies which have defective probability greater than 0.8 and tagged as fails by modified EM, but pass the downstream $10^{\circ} \mathrm{C}$ wafer-sort test are called as overkills. In Figure 4.12a, three dies are overkills while in Figure 4.12b, four dies are overkills. Dies having defective probability less than 0.8 and tagged as passes by modified $\mathrm{EM}$, but fail the downstream $10^{\circ} \mathrm{C}$ are called as test escapes. The two wafers in Figure 4.12 have zero test-escapes.

There is a trade-off between overkill and test escape based on the value of probability threshold. If the probability threshold is increased, less number of dies will be tagged as fails and overkills will be less but more defective dies will escape the test. In the case of lower probability threshold, more dies will be tagged as fails. However, more dies will be overkills but there will be less test escapes.

Figure 4.13 shows the defective probability plots of the two wafers of Product B. Figure $4.13 \mathrm{a}$ and $4.13 \mathrm{~b}$ show that there is a bunch of wafer-sort passing dies (triangles) in probability region from 0.5 to 0.6 . Most of the wafer-sort failing dies (squares) have probability greater than 0.85 . There is a remarkable gap in both wafer 1 and wafer 2 defective die probability plots separating the high and low probability dies. 


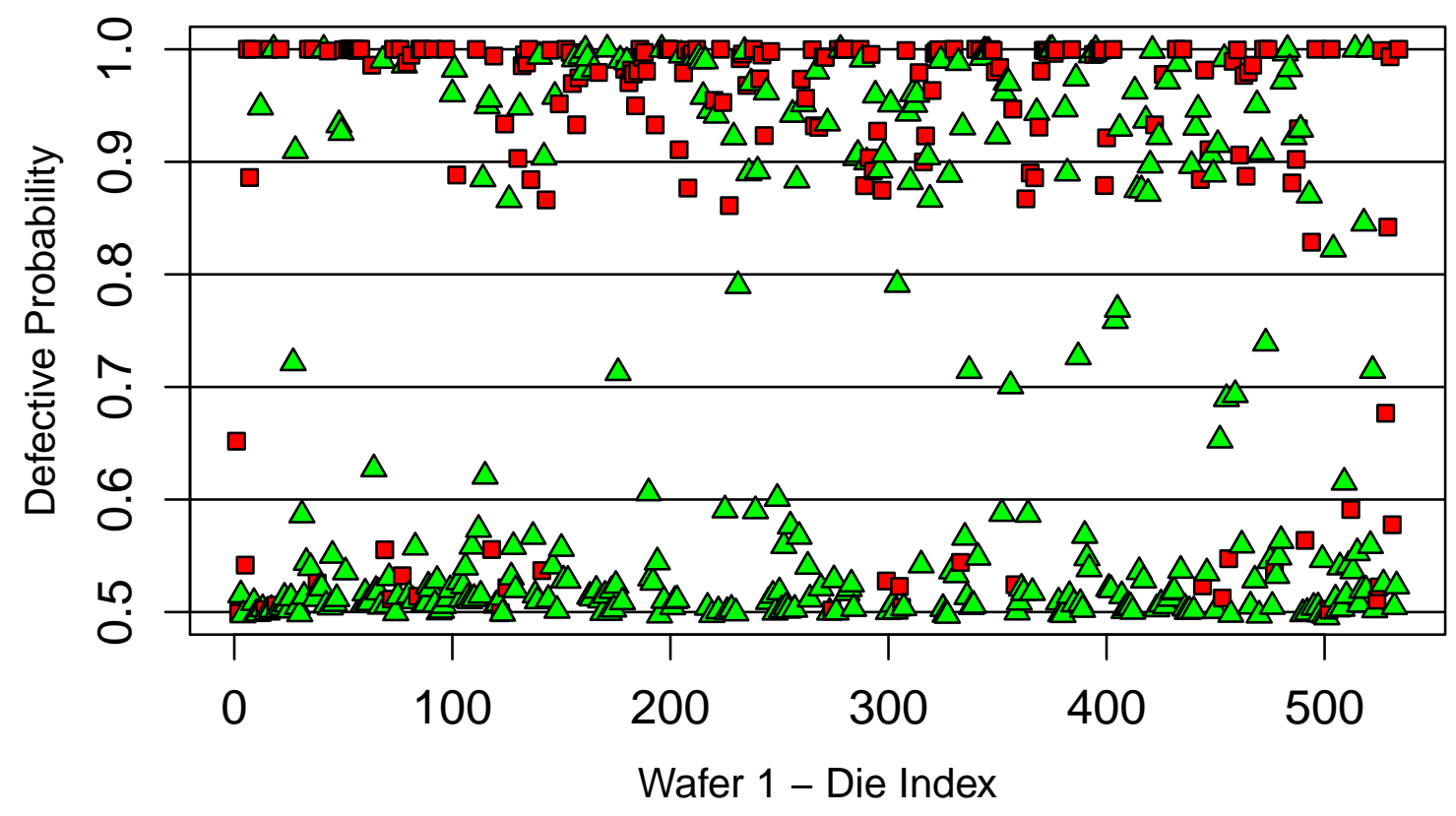

(a) Product B - wafer 1.

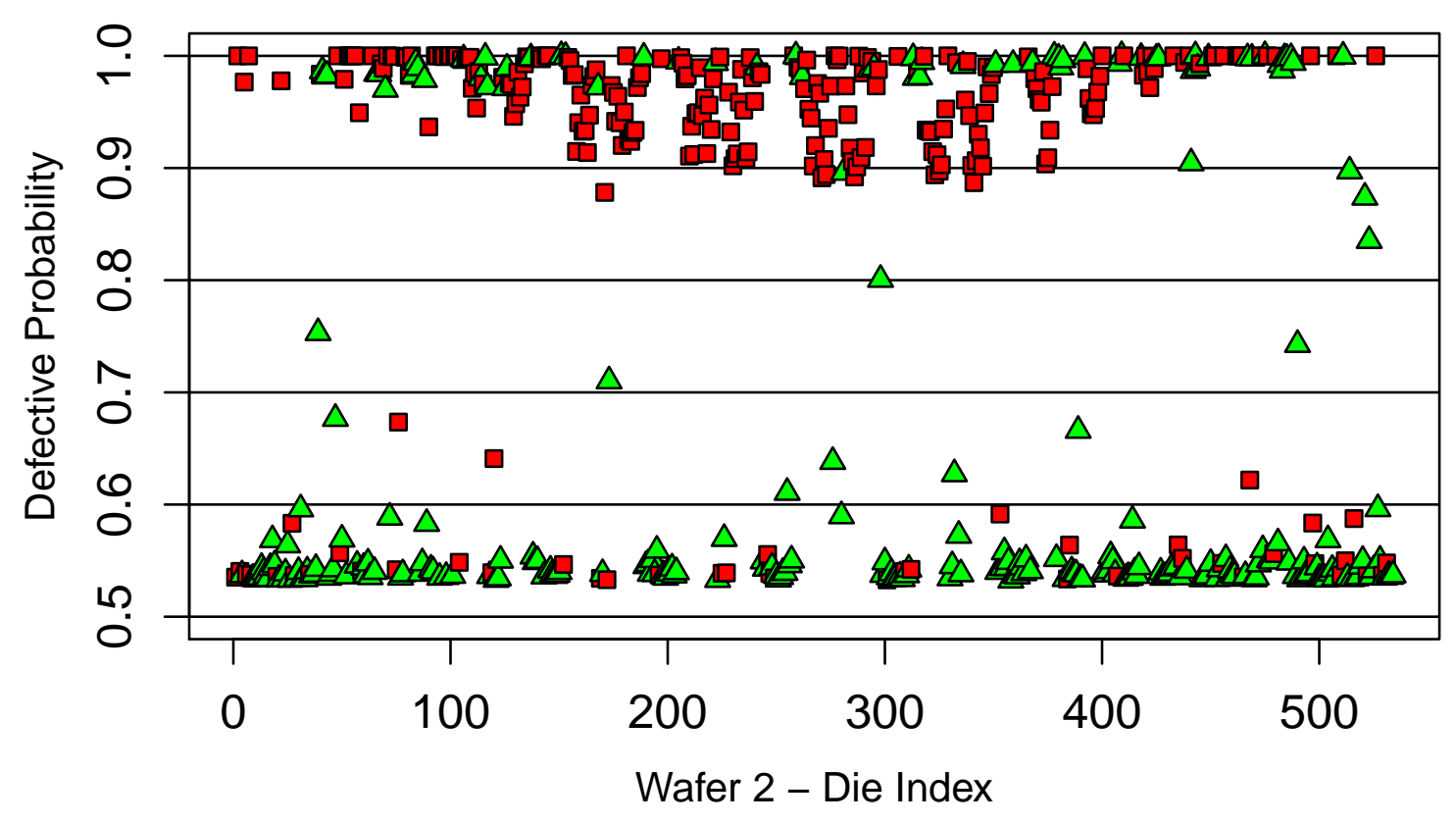

(b) Product B - wafer 2.

Figure 4.13: Defective probability plots of two wafers of Product B. Triangles are wafer-sort passes while squares are wafer-sort fails. 


\subsubsection{Key Figures of Merit}

Figure 4.14a shows the original wafer-sort test and Figure 4.14b shows the placement of modified EM method which acts as an improved screen in the test flow. The key figures of merit of modified EM method will be calculated by the dies tagged as passes and fails by modified EM method and comparing their results at $10^{\circ} \mathrm{C}$ wafer-sort test. Let's assume:

1. Killed dies mean the dies rejected by modified EM method.

2. Healthy dies mean $10^{\circ} \mathrm{C}$ wafer-sort passing dies.

3. Faulty dies mean $10^{\circ} \mathrm{C}$ wafer-sort passing dies

The overkills and test escapes of modified EM method are measured in terms of kill ratio and DPPM. Yield loss will be calculated to observe the impact of improved screen on yield. As shown in Figure 4.14, let's denote:

$$
\begin{aligned}
& a=\text { Total number of dies } \\
& b=\text { Total number of } 85^{\circ} \mathrm{C} \text { wafer-sort failing dies } \\
& c=\text { Total number of } 85^{\circ} \mathrm{C} \text { wafer-sort passing dies } \\
& d=\text { Total number of } 10^{\circ} \mathrm{C} \text { wafer-sort failing (Faulty) dies } \\
& e=\text { Total number of } 10^{\circ} \mathrm{C} \text { wafer-sort passing (Healthy) dies } \\
& p=\text { Total number of dies killed by modified EM } \\
& q=\text { Total number of dies not killed by modified EM }
\end{aligned}
$$

For original test flow, $c$ can be expressed as:

$$
c=d+e
$$




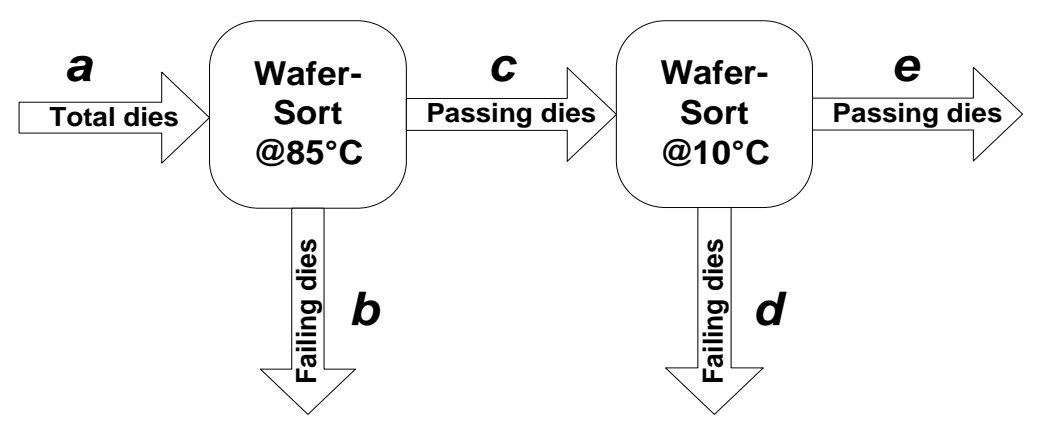

(a) Original wafer-sort test flow.

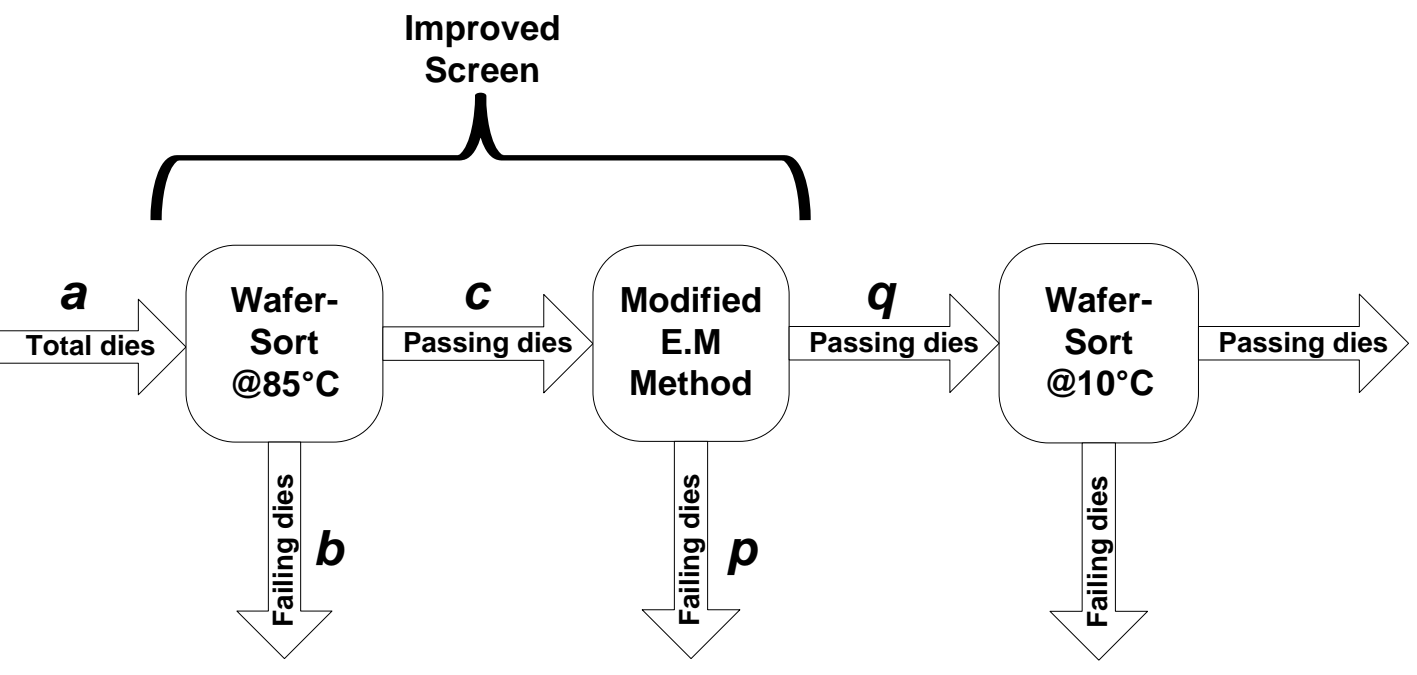

(b) Improved screen after placement of modified EM method in test flow.

Figure 4.14: Figure illustrating the original and modified test flow. 
For improved screen flow, $c$ can be expressed as:

$$
c=p+q
$$

Figure 4.15 shows a $2 \times 2$ contingency table. Dies which fail both $10^{\circ} \mathrm{C}$ test and modified EM method are called as common fails. Dies which are killed by modified EM method but pass $10^{\circ} \mathrm{C}$ test are overkills. Dies which are passes by modified EM method but fail in $10^{\circ} \mathrm{C}$ test are test escapes. Dies which are passes to both $10^{\circ} \mathrm{C}$ test and modified EM method are common passes.

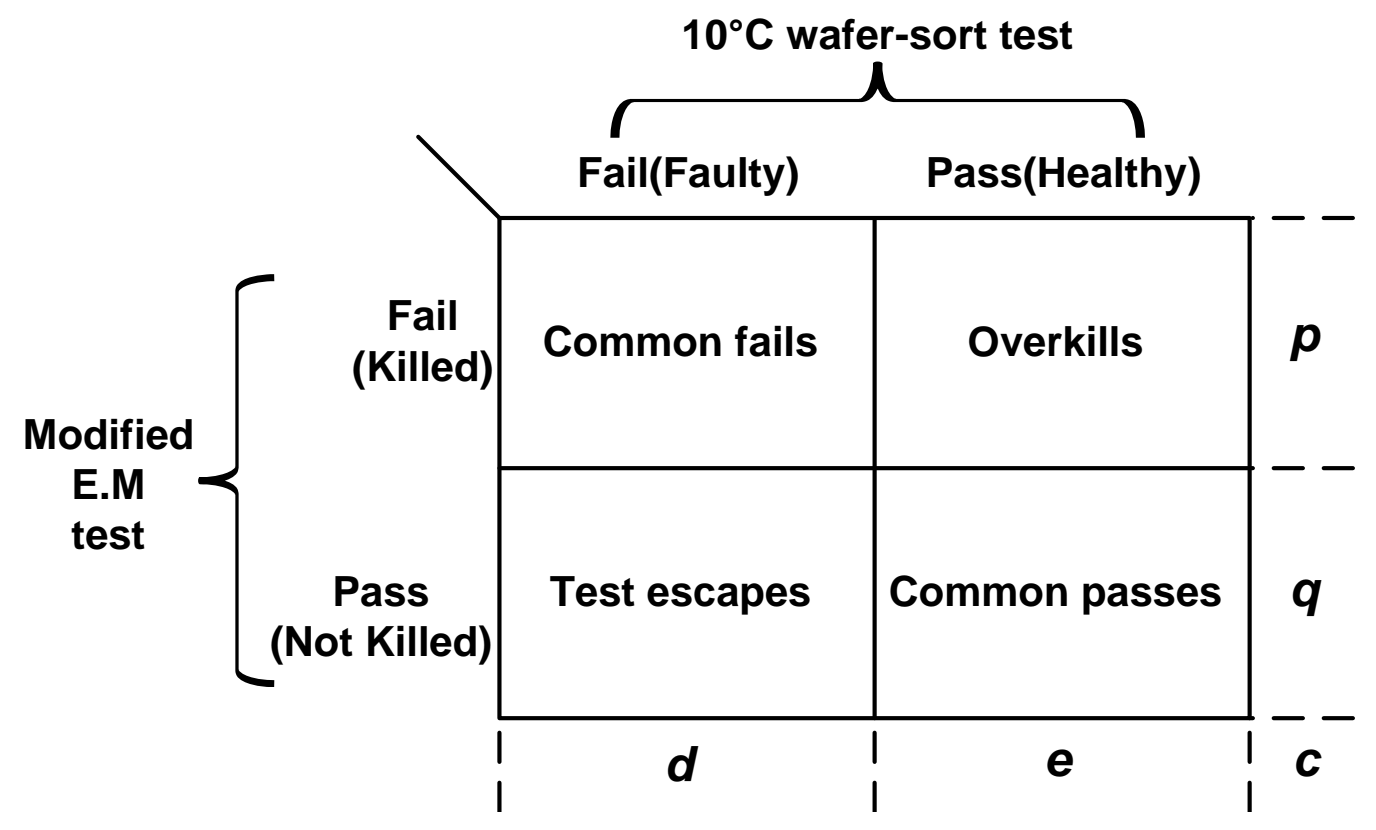

Figure 4.15: Calculating the figures of merit of modified EM method using 2 by 2 contingency table.

The $10^{\circ} \mathrm{C}$ wafer-sort failing dies represent dies which are failing stuck-at pattern tests, delay fails and IDDQ fails. There are other failing mechanisms of dies in Product A such as e-dram and ring oscillator fails which will not be considered in 
the kill ratio and DPPM calculations. The reason is that modified EM algorithm is applied to IDDQ data and hence other fail mechanisms cannot be compared with fails detected using IDDQ.

Kill ratio (KR) denotes the ratio of healthy dies killed for each defective die killed by the modified EM test. Higher kill ratio is a problem for test because more dies are thrown away at the cost of eliminating a defective die. It is calculated as:

$$
\mathrm{KR}=\frac{\text { Common Fails }}{\text { Overkills }}
$$

The improved screen reduces the number of defective dies passed to the downstream test. Using the contingency table, the DPPM is calculated for both original test flow and the improved screen flow. The difference between the DPPM of original test flow and modified test flow is DPPM reduction. Higher the DPPM reduction, more efficiently the method is screening the defective dies. It is calculated as:

$$
\begin{aligned}
\triangle \mathrm{DPPM} & =\text { Original Test Flow DPPM - Improved Screen DPPM } \\
& =\frac{d}{c}-\frac{d-\text { Common Fails }}{c} \\
& =\frac{\text { Common Fails }}{c}
\end{aligned}
$$

Yield loss should be as minimum as possible. The change in yield loss due to improved screen is calculated as:

$$
\begin{aligned}
\Delta \mathrm{YL} & =\text { Improved Screen YL - Original Test Flow YL } \\
& =\frac{p}{c}
\end{aligned}
$$


The tables 4.1, 4.2, 4.3 and 4.4 summarize the kill ratio of Product A for probability threshold value of 0.95 for Lots $1-4$. The bold numbers on the $\mathrm{x}$-axis of the tables represent the number of healthy dies killed by modified EM method. The bold numbers on the y-axis of the tables represent the number of faulty dies killed. If a bold number is picked from y-axis of table and another bold number is picked from $\mathrm{x}$-axis, the ratio of two bold numbers (y-axis/x-axis) denotes a kill ratio. The numbers in the table correspond to the number of wafers having a kill ratio of $\mathrm{y}$-axis/x-axis in a lot. For example, in Table 4.1, ten wafers in lot 1 have a kill ratio of 0/0. A kill ratio of 0/0 means that zero dies (healthy/faulty) are killed. For lot 1 , ten wafers fall in the $0 / 1$ kill ratio category. The kill ratio equal to $0 / 1$ means zero faulty dies are killed with one healthy die being killed. Kill ratio equal to $0 / n$ is the worst kill ratio for a test which does not kill any faulty die but kills $n$ healthy dies. In Table 4.1, two wafers have a kill ratio of $1 / 0$. Kill ratio equal to $1 / 0$ means that one faulty die is killed with zero healthy dies killed. Kill ratio equal to $n / 0$ is the ideal kill ratio of a test which kills $n$ faulty dies without killing any healthy die. There are other ranges of kill ratio in the tables.

\begin{tabular}{|l|l|l|l|l|l|l|l|l|l|}
\hline $\mathbf{2}$ & 0 & 0 & 0 & 0 & 0 & 0 & 0 & 0 & 0 \\
\hline $\mathbf{1}$ & 2 & 0 & 0 & 0 & 0 & 0 & 0 & 0 & 0 \\
\hline $\mathbf{0}$ & 10 & 10 & 2 & 1 & 0 & 0 & 0 & 0 & 0 \\
\hline & $\mathbf{0}$ & $\mathbf{1}$ & $\mathbf{2}$ & $\mathbf{3}$ & $\mathbf{4}$ & $\mathbf{5}$ & $\mathbf{6}$ & $\mathbf{7}$ & $\mathbf{8}$ \\
\hline
\end{tabular}

Table 4.1: Kill ratio of Lot 1 for probability threshold $=0.95$. Total $\mathrm{KR}=1 / 9$.

\begin{tabular}{|l|l|l|l|l|l|l|l|l|l|}
\hline $\mathbf{2}$ & 0 & 0 & 0 & 1 & 1 & 0 & 0 & 0 & 0 \\
\hline $\mathbf{1}$ & 0 & 0 & 1 & 1 & 3 & 0 & 0 & 0 & 0 \\
\hline $\mathbf{0}$ & 2 & 2 & 3 & 2 & 0 & 1 & 0 & 0 & 0 \\
\hline & $\mathbf{0}$ & $\mathbf{1}$ & $\mathbf{2}$ & $\mathbf{3}$ & $\mathbf{4}$ & $\mathbf{5}$ & $\mathbf{6}$ & $\mathbf{7}$ & $\mathbf{8}$ \\
\hline
\end{tabular}

Table 4.2: Kill ratio of Lot 2 for probability threshold $=0.95$. Total $\mathrm{KR}=1 / 5$. 


\begin{tabular}{|l|l|l|l|l|l|l|l|l|l|}
\hline $\mathbf{2}$ & 0 & 0 & 0 & 0 & 0 & 0 & 0 & 0 & 0 \\
\hline $\mathbf{1}$ & 1 & 1 & 2 & 0 & 0 & 0 & 0 & 0 & 0 \\
\hline $\mathbf{0}$ & 10 & 8 & 1 & 1 & 0 & 0 & 0 & 0 & 0 \\
\hline & $\mathbf{0}$ & $\mathbf{1}$ & $\mathbf{2}$ & $\mathbf{3}$ & $\mathbf{4}$ & $\mathbf{5}$ & $\mathbf{6}$ & $\mathbf{7}$ & $\mathbf{8}$ \\
\hline
\end{tabular}

Table 4.3: Kill ratio of Lot 3 for probability threshold $=0.95$. Total $\mathrm{KR}=1 / 5$.

\begin{tabular}{|l|l|l|l|l|l|l|l|l|l|}
\hline $\mathbf{2}$ & 0 & 0 & 0 & 0 & 0 & 0 & 0 & 0 & 0 \\
\hline $\mathbf{1}$ & 1 & 0 & 0 & 0 & 0 & 0 & 0 & 0 & 0 \\
\hline $\mathbf{0}$ & 22 & 0 & 0 & 0 & 0 & 0 & 0 & 0 & 0 \\
\hline & $\mathbf{0}$ & $\mathbf{1}$ & $\mathbf{2}$ & $\mathbf{3}$ & $\mathbf{4}$ & $\mathbf{5}$ & $\mathbf{6}$ & $\mathbf{7}$ & $\mathbf{8}$ \\
\hline
\end{tabular}

Table 4.4: Kill ratio of Lot 4 for probability threshold $=0.95$. Total $\mathrm{KR}=1 / 0$.

The kill ratio is aggregated for all wafers of a lot and shown as Total KR in the title of the corresponding table. For a probability threshold of 0.95 , kill ratio of lot 1 equal to $1 / 9$ is highest. Lot 4 has the lowest kill ratio of $1 / 0$. Lot 3 and 4 have a equal kill ratio of $1 / 5$. Lot 4 is having a low kill ratio because the tau distribution mixture calculated from each of 22 wafers falling in $0 / 0$ kill ratio category do not have enough separation between healthy and defective components for modified EM to resolve. The tau distribution looks like a single healthy distribution which results in all dies being tagged as healthy by modified EM and consequently having a low faulty probability.

\begin{tabular}{|l|l|l|l|l|l|l|l|l|l|}
\hline $\mathbf{2}$ & 0 & 0 & 0 & 0 & 0 & 0 & 0 & 0 & 0 \\
\hline $\mathbf{1}$ & 1 & 0 & 1 & 1 & 0 & 0 & 0 & 0 & 0 \\
\hline $\mathbf{0}$ & 3 & 4 & 7 & 6 & 0 & 1 & 1 & 0 & 0 \\
\hline & $\mathbf{0}$ & $\mathbf{1}$ & $\mathbf{2}$ & $\mathbf{3}$ & $\mathbf{4}$ & $\mathbf{5}$ & $\mathbf{6}$ & $\mathbf{7}$ & $\mathbf{8}$ \\
\hline
\end{tabular}

Table 4.5: Kill ratio of Lot 1 for probability threshold $=0.80$. Total $\mathrm{KR}=1 / 17$.

Table 4.5, 4.6, 4.7 and 4.8 shows the kill ratio of modified EM on Product A using a probability threshold of 0.8 . For lot 1 , the total kill ratio is $1 / 9$ as compared to kill ratio of $1 / 17$ when probability threshold of 0.8 is used. For lot 2 , the total kill ratio 


\begin{tabular}{|l|l|l|l|l|l|l|l|l|l|}
\hline $\mathbf{2}$ & 0 & 0 & 0 & 0 & 0 & 1 & 0 & 1 & 0 \\
\hline $\mathbf{1}$ & 0 & 0 & 2 & 1 & 0 & 1 & 2 & 0 & 1 \\
\hline $\mathbf{0}$ & 1 & 2 & 0 & 3 & 1 & 0 & 0 & 1 & 0 \\
\hline & $\mathbf{0}$ & $\mathbf{1}$ & $\mathbf{2}$ & $\mathbf{3}$ & $\mathbf{4}$ & $\mathbf{5}$ & $\mathbf{6}$ & $\mathbf{7}$ & $\mathbf{8}$ \\
\hline
\end{tabular}

Table 4.6: Kill ratio of Lot 2 for probability threshold $=0.80$. Total $\mathrm{KR}=1 / 6$.

\begin{tabular}{|l|l|l|l|l|l|l|l|l|l|}
\hline $\mathbf{2}$ & 0 & 0 & 0 & 0 & 1 & 0 & 0 & 0 & 0 \\
\hline $\mathbf{1}$ & 2 & 1 & 1 & 0 & 1 & 1 & 0 & 0 & 0 \\
\hline $\mathbf{0}$ & 5 & 6 & 5 & 0 & 1 & 0 & 0 & 0 & 0 \\
\hline & $\mathbf{0}$ & $\mathbf{1}$ & $\mathbf{2}$ & $\mathbf{3}$ & $\mathbf{4}$ & $\mathbf{5}$ & $\mathbf{6}$ & $\mathbf{7}$ & $\mathbf{8}$ \\
\hline
\end{tabular}

Table 4.7: Kill ratio of Lot 3 for probability threshold $=0.80$. Total $\mathrm{KR}=1 / 5$.

decreased from $1 / 6$ to $1 / 5$ when using a higher probability threshold of 0.95 . As the probability threshold is increased, less number of dies get killed by modified EM. Consequently, kill ratio decreases for increasing probability threshold. For lot 4 , the kill ratio almost remained constant for both threshold of 0.8 and 0.95 because no dies were above the probability threshold of 0.95 .

Figure 4.16 summarizes the DPPM reduction $(\triangle \mathrm{DPPM})$ and increase in yield loss $(\Delta \mathrm{YL})$ and overkill in percentage for the probability threshold $(\mathrm{PT})$ values of 0.95, 0.90, 0.85 and 0.80 for all lots in Product A. For lot 1, the DPPM reduction increases from $20 \%$ using probability threshold of 0.95 to $30 \%$ using probability threshold of 0.90. As the probability threshold is decreased, more dies will be killed by modified EM method and more faulty dies will come in the lower probability range. There is no change in DPPM from 0.90 to 0.80 because there are no dies

\begin{tabular}{|l|l|l|l|l|l|l|l|l|l|}
\hline $\mathbf{2}$ & 0 & 0 & 0 & 0 & 0 & 0 & 0 & 0 & 0 \\
\hline $\mathbf{1}$ & 2 & 1 & 0 & 0 & 0 & 0 & 0 & 0 & 0 \\
\hline $\mathbf{0}$ & 18 & 2 & 0 & 0 & 0 & 0 & 0 & 0 & 0 \\
\hline & $\mathbf{0}$ & $\mathbf{1}$ & $\mathbf{2}$ & $\mathbf{3}$ & $\mathbf{4}$ & $\mathbf{5}$ & $\mathbf{6}$ & $\mathbf{7}$ & $\mathbf{8}$ \\
\hline
\end{tabular}

Table 4.8: Kill ratio of Lot 4 for probability threshold $=0.80$. Total $\mathrm{KR}=1 / 1$. 
within the probability range of 0.90 to 0.80 which are faulty dies. For lot 1 , there is an average $27.5 \%$ reduction in DPPM level for all probability threshold values.For lot 2, average DPPM reduction is $42.7 \%$ which is significant amount of DPPM reduction. For lot 3 , there is jump of $11.6 \%$ in DPPM reduction as threshold is decreased from 0.95 to 0.90 which means a lot of faulty dies are in range of 0.95 to 0.90 . Lot 4 has an average DPPM reduction of $9.3 \%$.

Figure 4.16 also shows the corresponding increase in yield loss and overkill for all lots. The yield loss and overkill show a increasing trend as probability threshold is increased due to increased killing of dies by modified EM method. The average yield loss is lowest in lot 4 while highest for lot 2 . There is a trade-off between yield loss and DPPM reduction. Increasing yield loss is costly but the product quality depends upon DPPM reduction.

\subsubsection{Effect of Reduced IDDQ Input States}

IDDQ has a high observability which means a small number of input states will be enough to achieve adequate defect coverage. In the overall set of input states,

only some input states can activate a defect in the circuit. There can be a small subset of input states which can have a good mix of both elevated and non elevated states. Using smaller number of input states can separate the healthy and faulty components in the tau mixture distributions.

Figure 4.17 shows the two tau distribution from an example wafer in lot 4 . The healthy component density is shown in leftmost curve while the faulty component density is shown in rightmost curve. Figure 4.17a shows the tau distribution calculated using 64 IDDQ input states while Figure 4.17b shows the tau distribution 


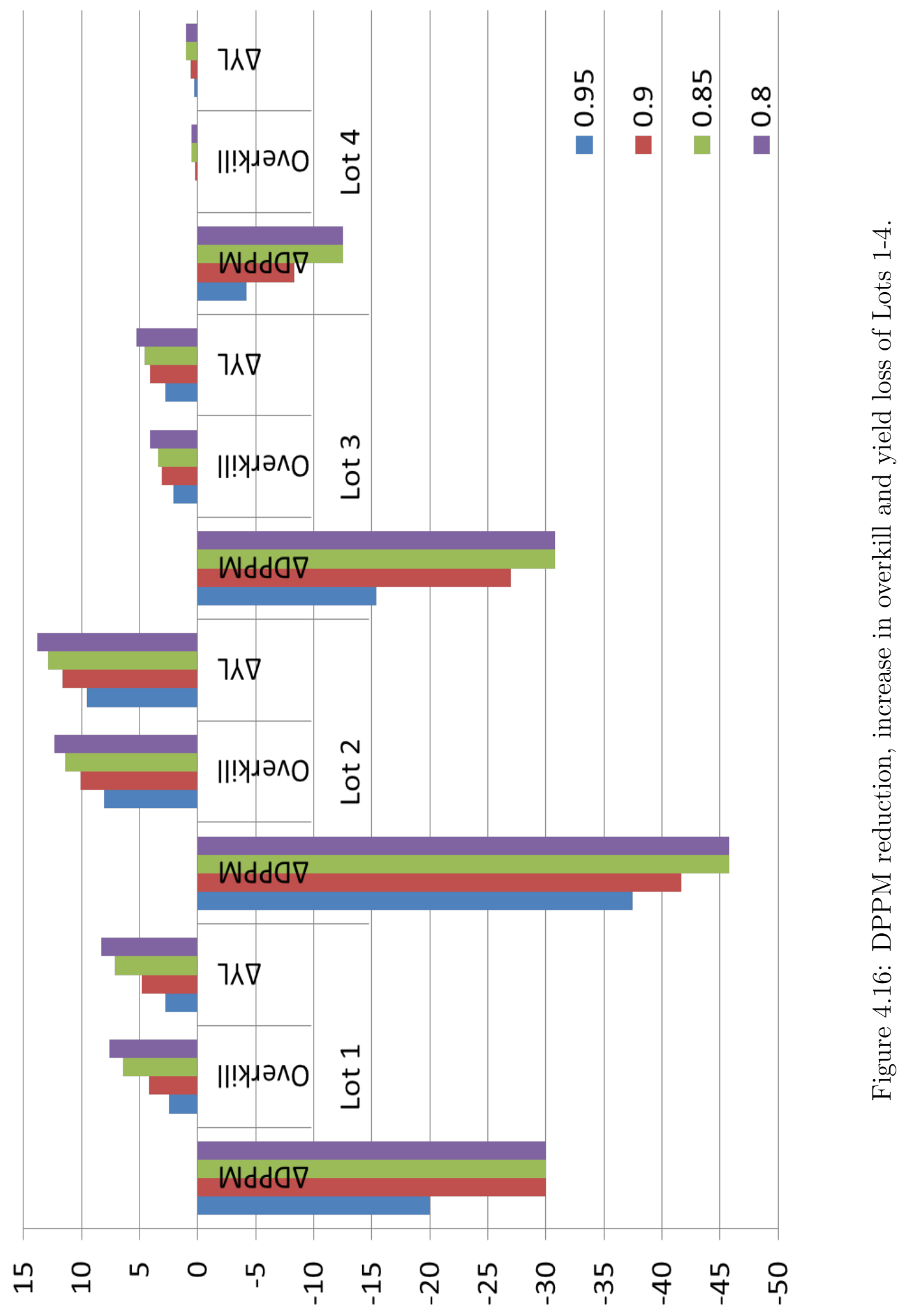


calculated for the same wafer using 16 IDDQ input states. In Figure 4.17b, the overlap between healthy and faulty components is much less as compared to overlap in Figure 4.17a. The group of 16 IDDQ states have a distributed mix of healthy and faulty IDDQ values which results in separation of the healthy and faulty tau components. The group of 64 IDDQ states for tau calculation has more healthy IDDQ values which results in mixing of faulty and healthy tau components.

For Product A, the 64 IDDQ input states can be divided into 2 groups of 32 states or 4 groups of 16 states or 8 group of 8 states. For each of the group, tau mixture distribution is calculated and given to the modified EM method. The modified EM calculates defective probability of each die. DPPM reduction using the modified EM method is calculated for each of the IDDQ states subset.

Figure 4.18 and 4.19 shows four discordance plots between test ranks of two dies from lot 1 in Product A. The discordance is calculated using 1st, 2nd, 3rd and 4th groups of eight IDDQ states. The number of crossings in the discordance plot is equal to the discordant pairs. More the number of discordant pairs, less will be the tau value between two test states. Figure 4.19a shows that the number of crossings are maximum in comparison of 3rd group of 8 IDDQ states for the two dies. Only the comparison of 3rd group of IDDQ vectors for two dies is able to catch the defect. The 1st, 2nd and 4th group of IDDQ vectors shows high correlation between test states for die 1 and die 2 . The die 1 failed in $10^{\circ} \mathrm{C}$ IDDQ test.

Figure 4.20 summarizes the DPPM results for the two probability thresholds of 0.8 and 0.95 . Figure 4.20 a shows the DPPM reduction in $\%$ for all the groups of 


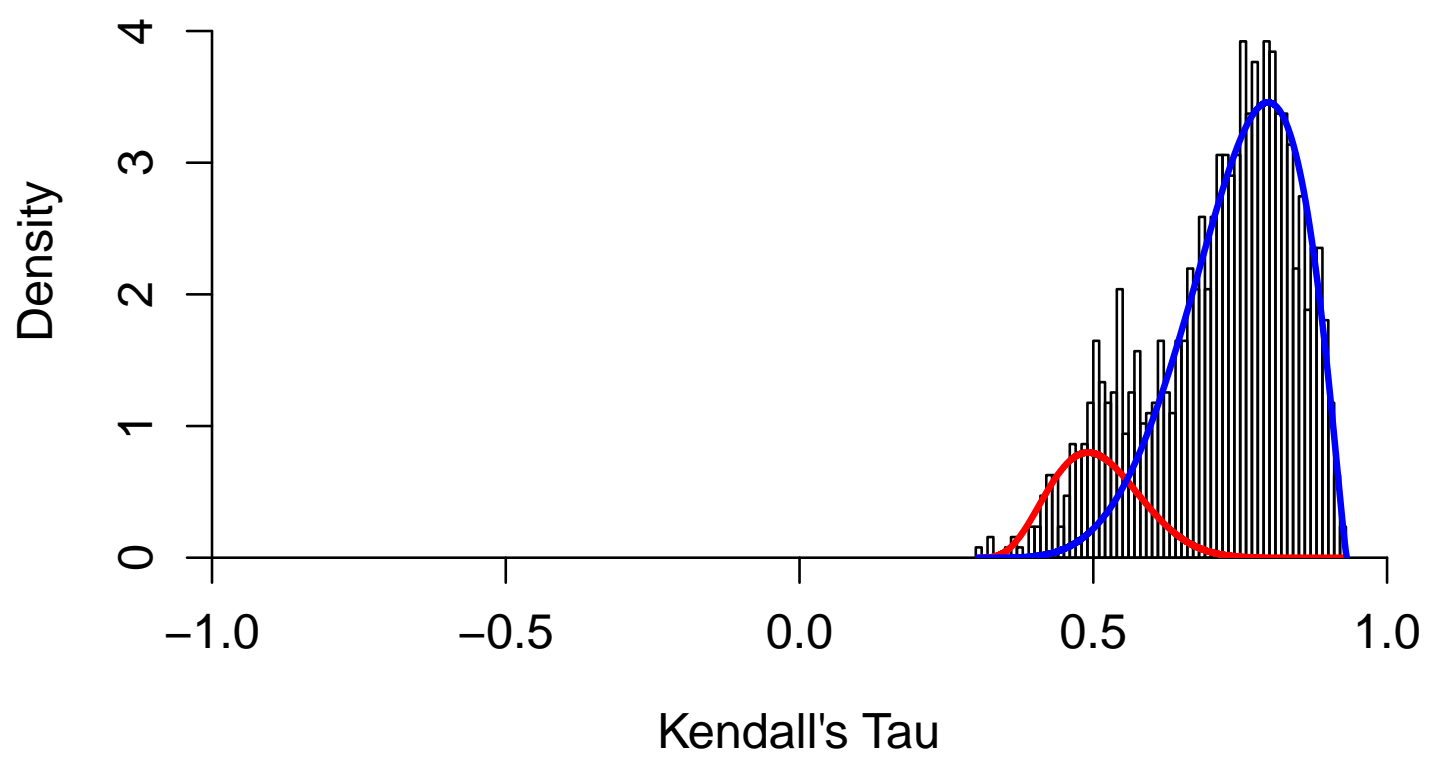

(a) Tau distribution calculated from 64 IDDQ states.

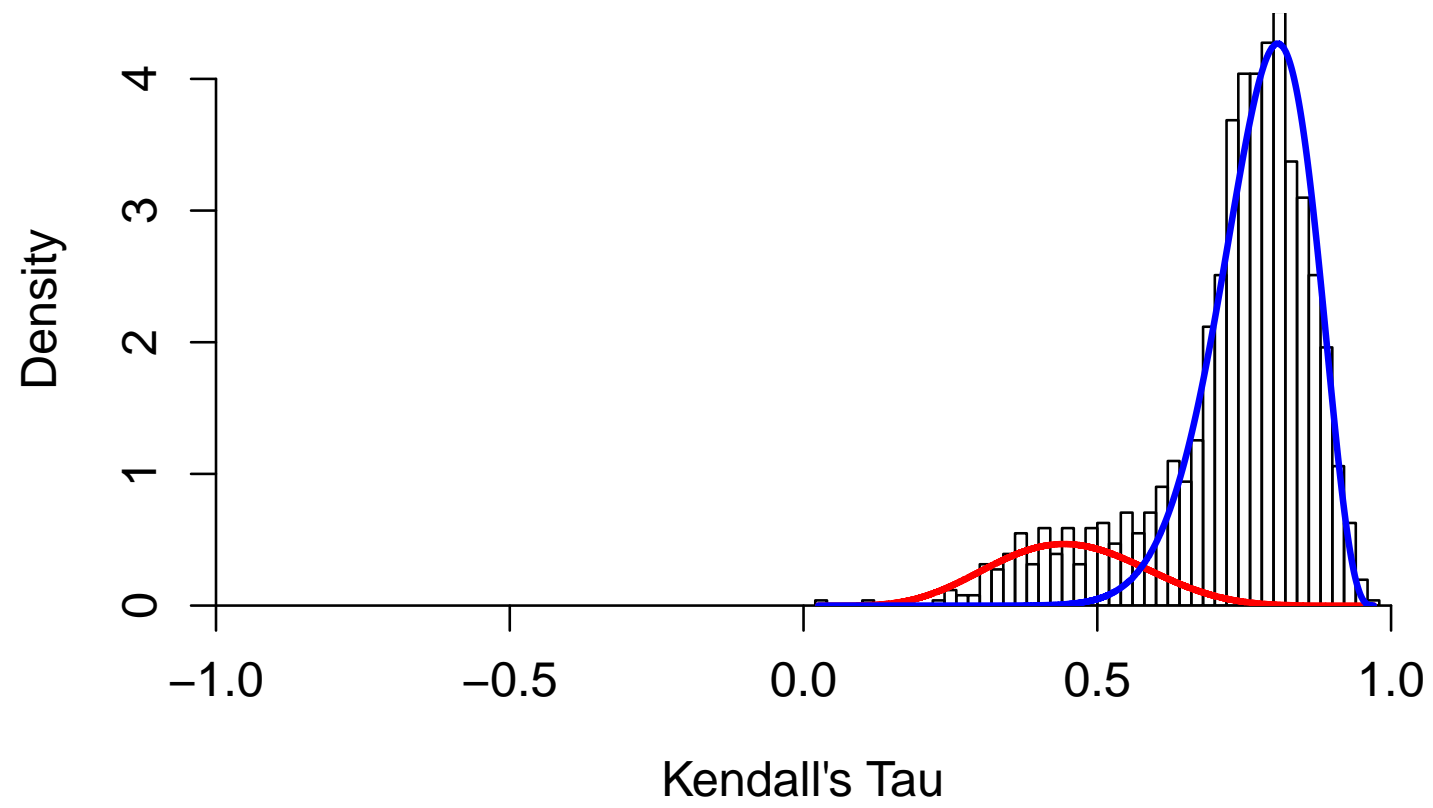

(b) Tau distribution calculated from 16 IDDQ states.

Figure 4.17: Tau distributions showing comparing the overlap of healthy and faulty components using 64 IDDQ states and 16 IDDQ states. 


\section{Ranked State Order of Die 2}

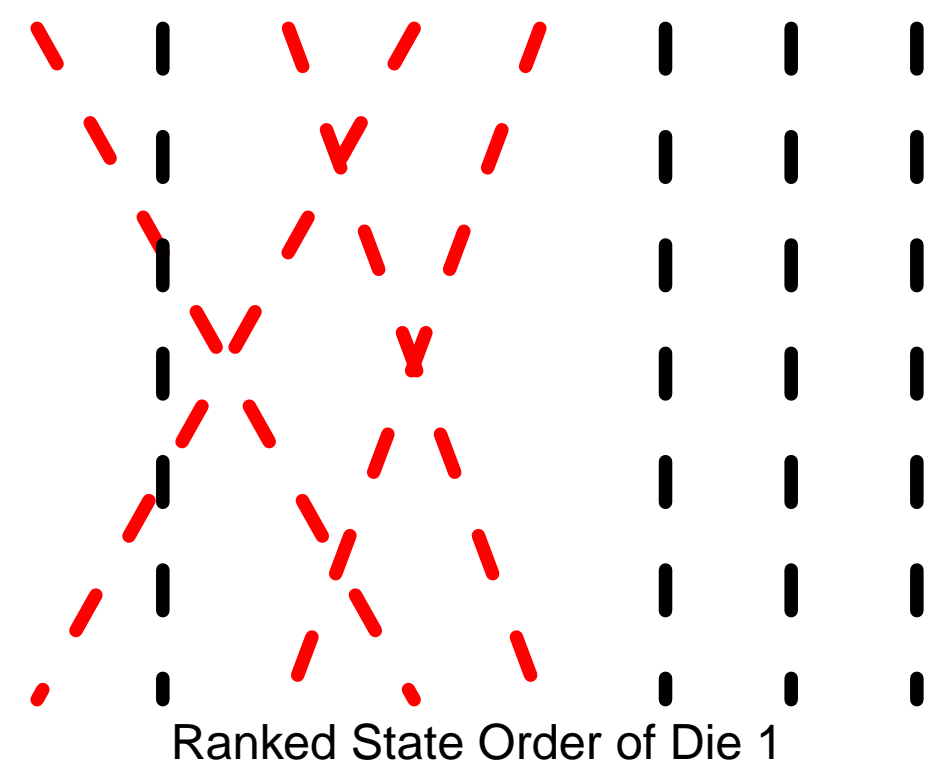

(a) 1st group of 8 states.

\section{Ranked State Order of Die 2}

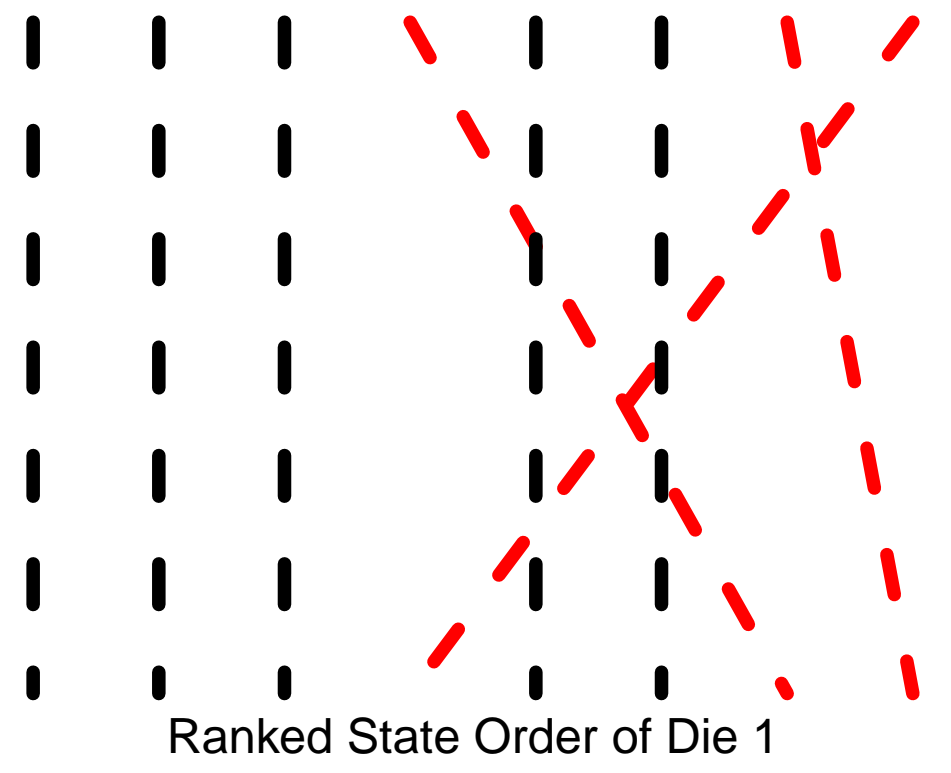

(b) 2nd group of 8 states.

Figure 4.18: Discordance plots of test ranks using 1st and 2nd group of eight IDDQ states of two dies from lot 1 in Product A. 
Ranked State Order of Die 2

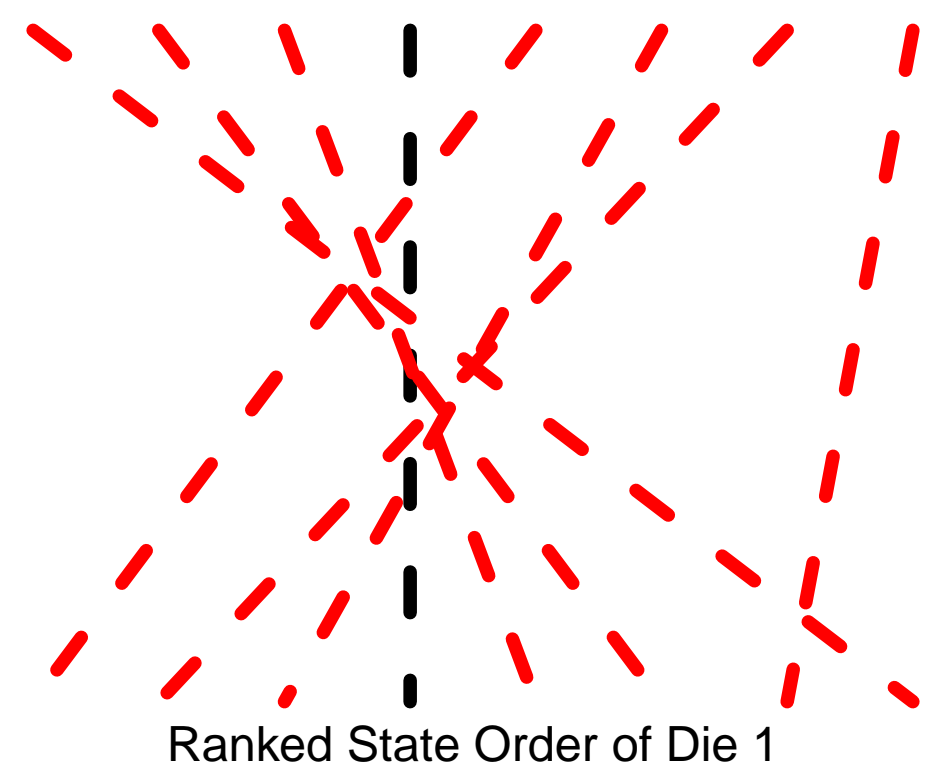

(a) 3rd group of 8 states.

\section{Ranked State Order of Die 2}

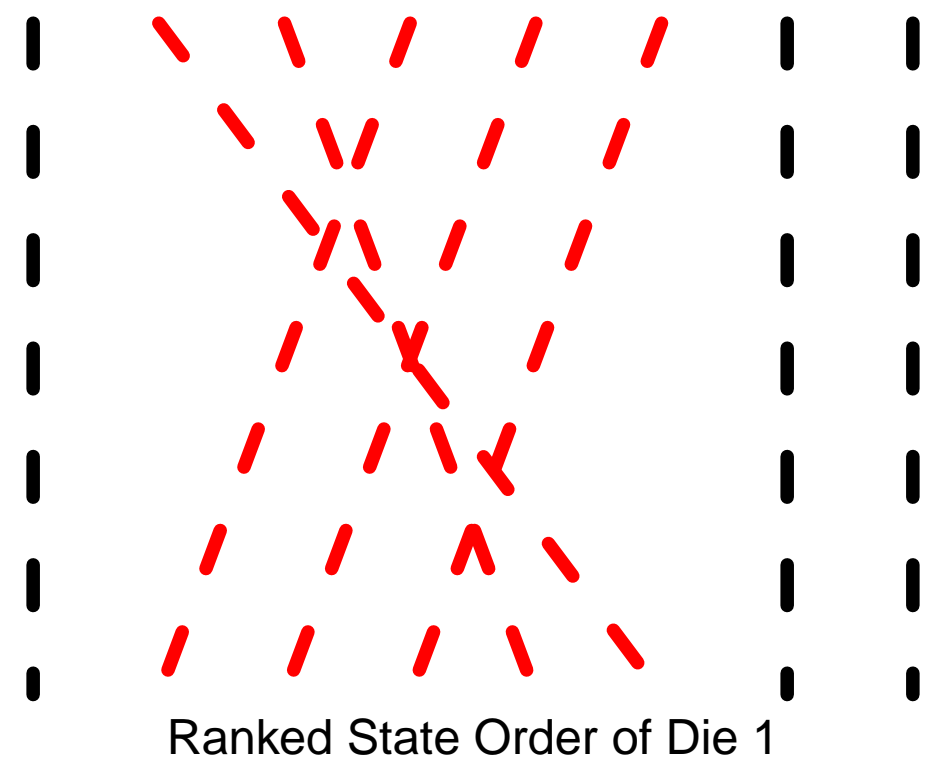

(b) 4th group of 8 states.

Figure 4.19: Discordance plots of test ranks using 3rd and 4th group of eight IDDQ states of two dies from lot 1 in Product A. 
IDDQ states using probability threshold of 0.8 . The 1 st subset of 32 states gives the highest average DPPM reduction which is more than the DPPM reduction obtained using 64 IDDQ states. Lot 2 has the highest DPPM reduction with 50\%. The DPPM reduction numbers get lower for subset of 16 IDDQ states. Among the group of 8 vectors, 4th group has the highest DPPM reduction. Similarly using probability threshold of 0.95 , DPPM reduction is plotted for all the groups of IDDQ states in Figure 4.20b. As the probability threshold is increased, it is expected to have less DPPM reduction because less dies will be killed by modified EM. For 0.95, the whole graph gets lowered when compared to graph of 0.80 . The DPPM reduction is again highest using 1st subset of 32 IDDQ states. In the group of 8 IDDQ states, 4th group has the highest DPPM reduction.

For maximum screening of defective dies, 1st 32 group of IDDQ states performs the best among the other groups. 


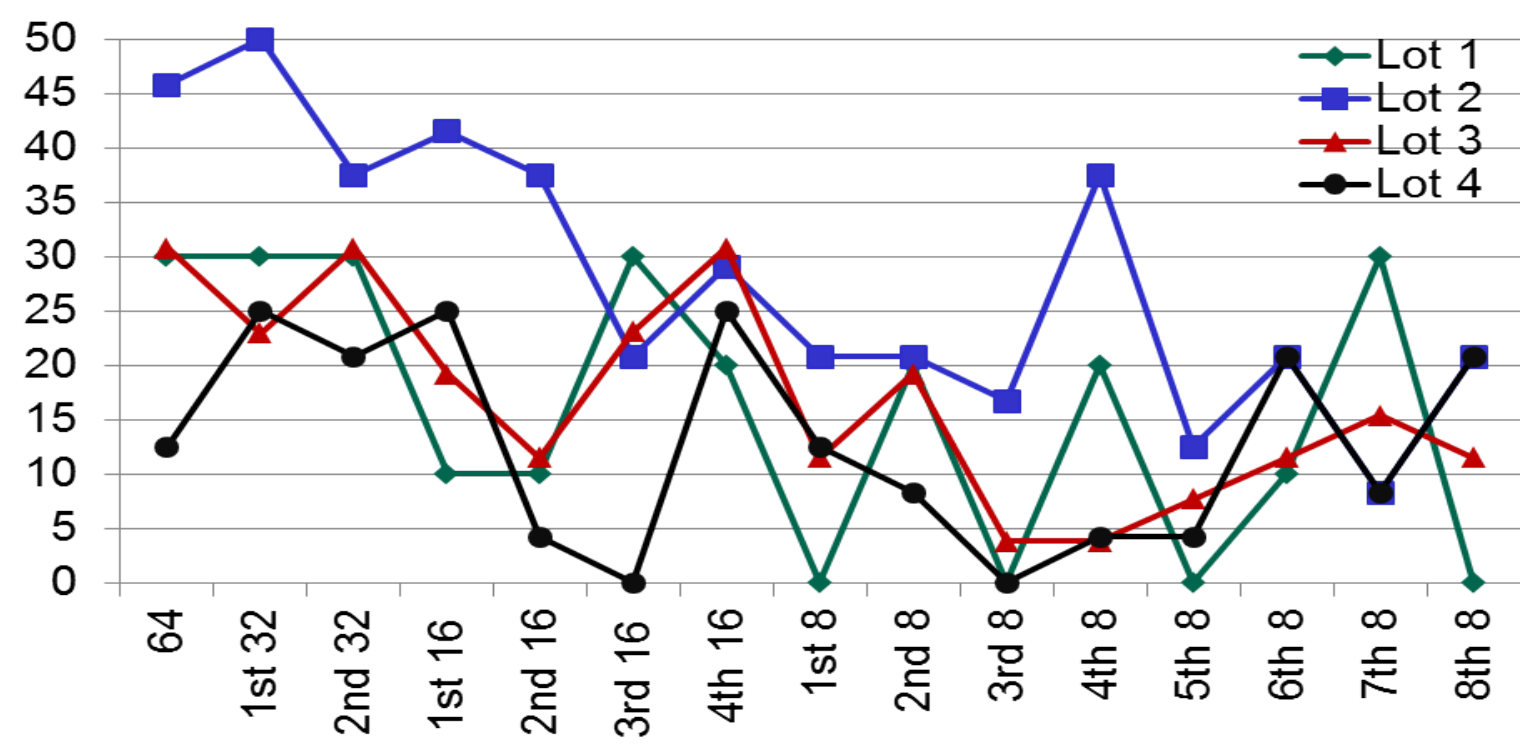

(a) Probability threshold of 0.80 .

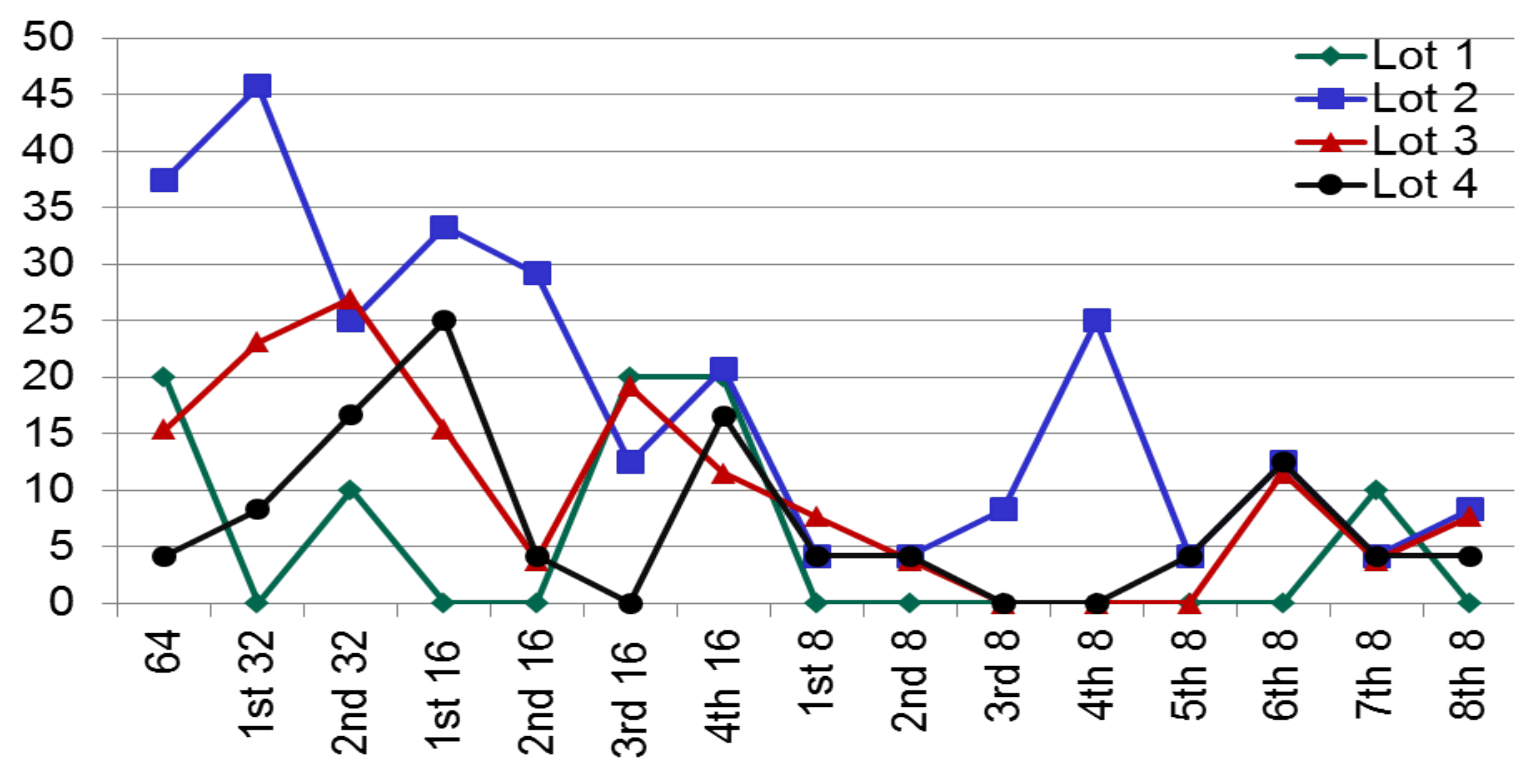

(b) Probability threshold of 0.95 .

Figure 4.20: DPPM reduction plots using defective die probabilities obtained from a reduced subset of IDDQ states. The dies above the probability threshold 0.80 and 0.95 are tagged as defective. 


\section{Chapter 5}

\section{Conclusion and Recommendations}

\subsection{Conclusion}

This thesis for the first time presents an inter-die rank screen which effectively screens out the defective die. The inter-die rank screen utilizes the change in rank-order of defective dies as compared to the consistent rank-order of healthy dies. The inter-die rank screen does not require the prior knowledge of rank of test states. There is no assumption for a wafer having a fixed order of a healthy parametric test rank-order which will be used to compare against the rank-order of defective dies.

The probability plots show there is a clear separation of healthy dies from faulty at wafer-sort as diagnosed by modified EM method. Thus, instead of putting individual limits on each of the parametric test states, the inter-die rank screen gives one number to differentiate between healthy and faulty dies. The inter-die rank screen solves the parametric limit setting problem by reducing the effects of process variation. The screen detects downstream defective die at wafer-sort.

There is a trade-off between kill rate and DPPM improvement. As the probability threshold of tagging a defective die is lowered, DPPM reduction takes place but the kill rate also increases. The inter-die rank screen works also on a reduced subset of input states if the states contain both elevated and non elevated vectors. If the reduced subset contains only elevated or only non elevated vectors, then the

correlation among all the dies will be high and defective probability of all the dies 
will be low. There is a reduction in kill rate but then the DPPM improvement also decreases.

The inter-die rank screen is insensitive to defective dies in case of passive defects, systematic defects and process shifts. A passive defect will activate all the vectors and hence the rank order of a defective die will be same as the healthy rank order. A systematic defect can result in higher tau values becoming a part of healthy distribution. The inter-die rank screen also won't work if the parametric test variations in a wafer are random and not in monotonic fashion. The test data in that case is not rank-able.

In the Monte Carlo simulations, there is a scenario where rounding of tau values will not cover the reduced number of input states problem. If tau is calculated from a subset of reduced input states which contain a proportion of both elevated and non elevated vectors, then the separation between healthy and faulty distribution will be good and hence there will not be a resolution problem. However if the subset contains only elevated or non elevated vectors, then the resolution problem will kick in for the modified EM method because there won't be enough resolution to separate between healthy and faulty distributions.

\subsection{Recommendations}

The modified EM method can be applied on any parametric test data such as minVdd or path delay. MinVDD of each die can be ranked at different frequencies and correlation can be utilized to screen defective dies. Path delay depends upon the input states. Critical path delay is measurable at wafer-sort test. For a die, maximum path delay from different critical paths can be measured for a specific 
state transition. A die will have path delay values for different state transitions. Tau can be calculated for inter-die pairs using different state transition path delay values. Correlation model can thus be build for the path delay using the same concept as IDDQ. Figure 5.1 shows the variation of path delays of 7 different critical paths on a die across the wafer. For a die, correlation can be calculated between inter-die pairs using maximum of seven path delays for a state transition and a correlation model similar to IDDQ correlation model can be built.

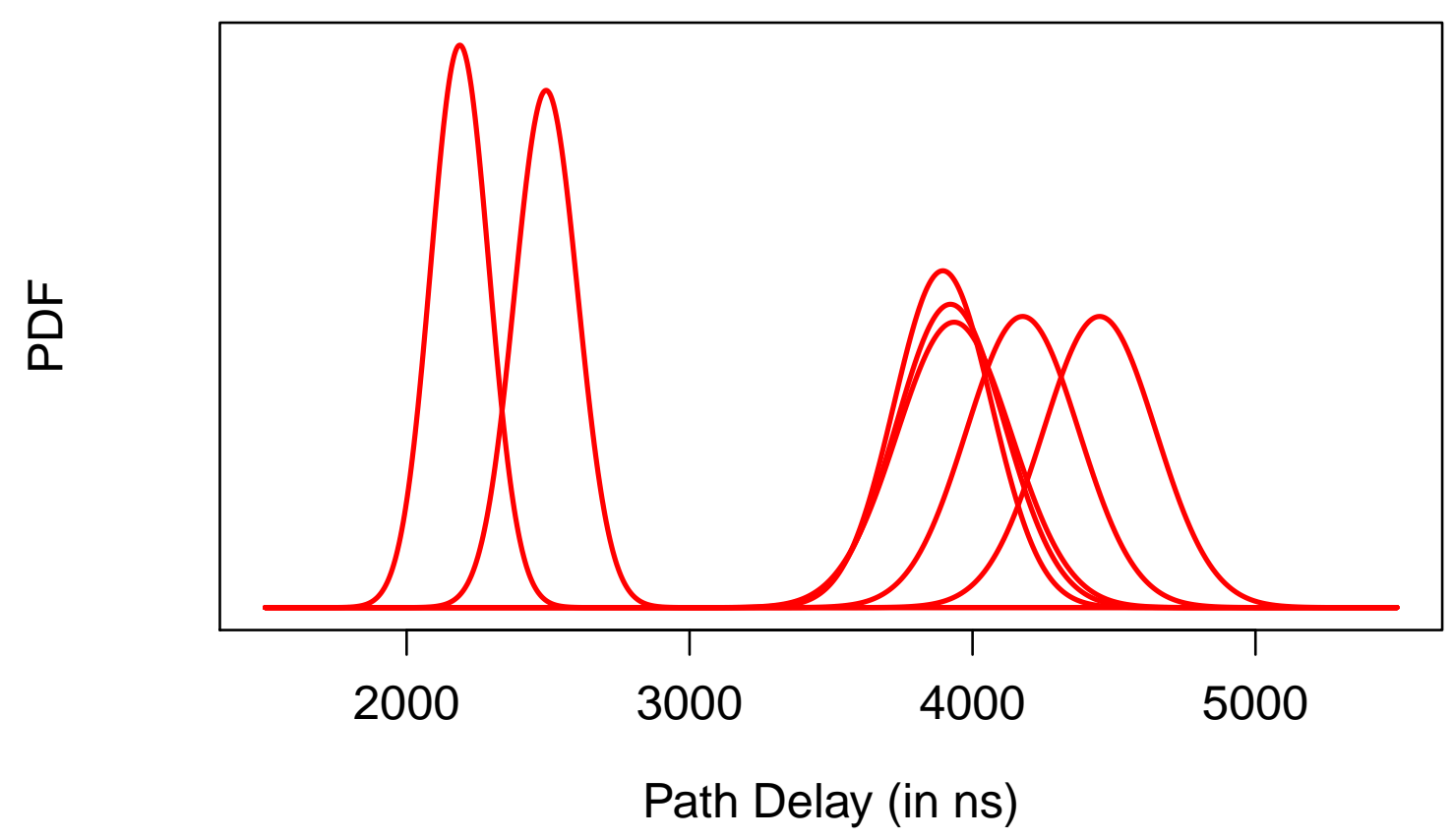

Figure 5.1: Variation of path delays of 7 different critical paths on a die across wafer [1].

Monte Carlo simulations show the miss-correlation between estimated parameters of mixture distribution in presence of a noisy beta distribution. The tau distribution calculated from production data has noise such as process noise and tester noise. If the noise in the tau calculated from production data can be modelled as a 
third beta distribution and subtracted from the tau mixture, more clear separation can be introduced between healthy and faulty dies. This can help in reducing the kill rate of the modified EM method.

My contribution to this thesis was to modify the existing code of EM algorithm and modify it to introduce the correlation model. The Monte Carlo simulator was also built to simulate the synthesized data. This thesis was presented as a poster in IEEE International Test Conference 2011 [19]. 


\section{References}

[1] K. Bowman, S. Duvall, and J. Meindl, "Impact of die-to-die and withindie parameter fluctuations on the maximum clock frequency distribution for gigascale integration," IEEE Journal of Solid-State Circuits, Feb 2002, vol. 37, no. 2 , pp. $183-190$.

[2] M. L. Bushnell and V. D. Agrawal, Essentials of Electronic Testing for Digital, Memory, and Mixed-Signal VLSI Circuits. Boston: Kluwer Academic, 2000, ch. 3, pp. $20-21$.

[3] C.-W. Tseng, E. Mccluskey, X. Shao, and D. Wu, "Cold delay defect screening," in Proceedings of 18th IEEE VLSI Test Symposium, May 2000, pp. 183 $-188$.

[4] F. Guess, E. Walker, and D. Gallant, "Burn-in to improve which measure of reliability?" Microelectronics Reliability, vol. 32, no. 6, pp. 759 - 762, 1992. [Online]. Available: http://www.sciencedirect.com/science/article/pii/ $002627149290039 \mathrm{~N}$

[5] L. C. Wagner, Failure Analysis of Integrated Circuits: Tools and Techniques. Southport: Chapman and Hall, 1999, ch. 2, p. 25.

[6] A. Gattiker and W. Maly, "Current signatures [vlsi circuit testing]," in Proceedings of 14th IEEE VLSI Test Symposium, May 1996, pp. 112 -117. 
[7] C. J. Lin and S. Reddy, "On delay fault testing in logic circuits," IEEE Transactions on Computer-Aided Design of Integrated Circuits and Systems, Sept 1987, vol. 6 , no. 5, pp. $694-703$.

[8] C.-W. Tseng, R. Chen, P. Nigh, and E. McCluskey, "Minvdd testing for weak cmos ics," in Proceedings of 19th IEEE VLSI Test Symposium, May 2001, pp. $339-344$.

[9] M. Ono, H. Iwata, and K. Watanabe, "Accuracy of yield impact calculation based on kill ratio," in Advanced Semiconductor Manufacturing Conference and Workshop, Aug 2002, pp. 87 -91.

[10] J. D. Gibbons, Nonparametric measures of association. Newbury Park: Sage Publications, 1993, ch. 2, pp. $3-10$.

[11] "Kendall tau rank correlation coefficient," http://en.wikipedia.org/wiki/ Kendall_tau_rank_correlation_coefficient,

[12] J. Podani, "A measure of discordance for partially ranked data when presence/absence is also meaningful," Coenses, 199\%, vol. 12, pp. $127-130$.

[13] E. Marinissen, A. Singh, D. Glotter, M. Esposito, J. Carulli, A. Nahar, K. Butler, D. Appello, and C. Portelli, "Adapting to adaptive testing," in Design, Automation Test in Europe Conference Exhibition (DATE), March 2010, pp. $556-561$.

[14] W. Daasch and R. Madge, "Variance reduction and outliers: statistical analysis of semiconductor test data," in Proceedings of IEEE International Test Conference, Nov 2005. 
[15] C. Thibeault, "On the comparison of delta iddq and iddq testing," in Proceedings of 17th IEEE VLSI Test Symposium, Apr 1999, pp. 143 -150.

[16] R. Gudavalli, W. Daasch, P. Nigh, and D. Heaberlin, "Application of nonparametric statistics of the parametric response for defect diagnosis," in Proceedings of IEEE International Test Conference, Nov 2009, pp. 1-10.

[17] S. Nadarajah and S. Kotz, "Two generalized beta distributions," Applied Economics, vol. 39, no. 14, pp. 1743-1751, 2007. [Online]. Available: http://www.tandfonline.com/doi/abs/10.1080/00036840500428062

[18] Y. Ji, C. Wu, P. Liu, J. Wang, and K. R. Coombes, "Applications of beta-mixture models in bioinformatics," Bioinformatics, vol. 21, pp. 2118 -2122, May 2005. [Online]. Available: http://dl.acm.org/citation.cfm?id= 1093777.1094109

[19] V. Bakshi and W. Daasch, "Application of inter-die rank statistics in defect detection," in Proceedings of IEEE International Test Conference, Sept 2011, no. PO-11. 